\title{
Nucleosynthesis of light elements inside thermally pulsing AGB stars
}

\section{The case of intermediate-mass $\operatorname{stars}^{\star}$}

\author{
M. Forestini ${ }^{1}$ and C. Charbonnel ${ }^{2}$ \\ 1 Laboratoire d'Astrophysique, Observatoire de Grenoble, Université Joseph Fourier, BP 53, F-38041 Grenoble Cedex 9, France \\ ${ }^{2}$ Laboratoire d'Astrophysique de Toulouse, CNRS UMR 5572, Toulouse, France
}

Received April 22; accepted July 30, 1996

\begin{abstract}
The structural and nucleosynthetic evolution of $3,4,5,6$ and $7 M_{\odot}$ stars with two metallicities $(Z=$ 0.005 and 0.02 ) has been computed in detail, from the early pre-main sequence phase up to the thermally pulsing (TP) AGB phase or the onset of off-center carbon burning. Typically 10 to 20 thermal pulses have been followed for each TP-AGB object. This homogeneous and quite large set of models allows us to present an overview of the thermal pulse properties as well as of the nucleosynthesis accompanying the TP-AGB phase of intermediate-mass stars.

More specifically, after a brief description of the previous evolutionary stages, predictions are given for the isotopic ratios involving $\mathrm{C}, \mathrm{N}, \mathrm{O}, \mathrm{Ne}, \mathrm{Mg}, \mathrm{Al}$ and $\mathrm{Si}$. Also the surface abundances of ${ }^{7} \mathrm{Li},{ }^{19} \mathrm{~F}$ and ${ }^{23} \mathrm{Na}$ are reported. As the asymptotic phase of the thermal pulses has been reached for each star, we also indicate how these abundances will probably evolve until the stars completely loose their envelope, by including the evolution of the nucleosynthesis itself.
\end{abstract}

Key words: stars: AGB — stars: interiors nucleosynthesis - stars: abundances

\section{Introduction}

At the end of their life, stars of low- and intermediatemass evolve along the Asymptotic Giant Branch, where they experience recurrent thermal instabilities and substantial mass loss. During this phase, the stars undergo a

Send offprint requests to: M. Forestini

* This article, in its paper form, has been shortened at a level of roughly $60 \%$ as required by directives coming from the A\&A editors. The complete article (50 pages containing 37 figures) is only available in electronic form. very rich and unique nucleosynthesis. Moreover, recurrent dredge-up events enrich the stellar surface with the freshly synthesized nuclides which are then ejected into the interstellar medium through the strong winds. The thermally pulsing AGB stars thus play a crucial role in the chemical evolution of galaxies.

On the other hand, AGB stars are important contributors to the integrated luminosity of stellar systems in a wide range of ages. A coverage of this phase is needed in particular for the treatment of the luminosity functions and color magnitude diagrams of star clusters. Last but not least, the corresponding stellar models are basic tools to study carbon stars, $\mathrm{OH} / \mathrm{IR}$ stars, planetary nebulae and their central objects (white dwarfs).

In the last 15 years, extensive observational data has become available that changed and highly constrained the theoretical view of the AGB evolution. Most of the progresses came from Magellanic Cloud studies, which revealed for example the absence of very luminous carbon stars (which were previously predicted to originate from stars with initial masses between 5 and $\left.8 M_{\odot}\right)$, and the unexpected existence of relatively faint and lower mass carbon stars (Blanco et al. 1980; Mould \& Aaronson 1982, 1986; Wood et al. 1983; Reid \& Mould 1984; Aaronson \& Mould 1985; Smith \& Lambert 1989). This so-called "carbon star mystery" (Iben 1981) has led to the concept of hot-bottom convective envelope burning (Iben 1975; Sackmann et al. 1974; Scalo et al. 1975; Sackmann \& Boothroyd 1992). This mechanism, which reconverts Crich to O-rich envelopes and produces ${ }^{7} \mathrm{Li}$, was confirmed by the existence of lithium enriched AGB stars (Smith \& Lambert 1989, 1990b; Plez et al. 1993; Smith et al. 1995). On the other hand, the discovery, inside meteorites, of interstellar grains which have been formed in the wind of cool carbon stars (see e.g. Zinner et al. 1991a), complemented the stellar abundance analysis, and brought crucial informations on AGB star nucleosynthesis. In spite of 
important improvements, many important issues of AGB evolution still remain to be addressed, observationally as well as theoretically.

This paper is the first of a series aimed at examining all the physical processes that may induce abundance anomalies in the surface of evolved AGB stars. In the present work, we specifically investigate the case of intermediate-mass stars. Stars with initial masses between 3 and $7 M_{\odot}$ and with metallicities $Z$ of 0.02 and 0.005 have been evolved, starting from the pre-main sequence until they had gone through a number of helium-shell flashes along the TP-AGB. The $7 M_{\odot}\left(6\right.$ and $\left.7 M_{\odot}\right)$ stars with $Z=0.02(0.005)$ ignite off-center carbon burning and will not be discussed in detail. For each other stellar mass, full evolutionary models were computed up to the fourth "full amplitude" thermal pulse in the so-called "asymptotic regime". Synthetic evolution was then used to follow global surface properties and nucleosynthesis up to the planetary nebula ejection. This self-consistent and extended set of models is particularly relevant for population synthesis and chemical evolution models. Very complete figures and tables containing informations about the center and surface evolution of the structure and chemical composition for all our models are available.

In the present computations, which include the latest input physics, the evolution of the chemical composition is followed by only taking into account nuclear reactions and mixing inside convective zones (we assume instantaneous mixing, except when hot-bottom burning occurs, which requires a time-dependent convective diffusion algorithm). We neglect all other mixing mechanism of non-convective origin which may contribute to transport chemical elements in radiative regions, like rotationinduced mixing or diffusion. In the stellar mass range we consider, this approximation is supported by the observed surface abundances at different evolutionary stages. This is however certainly not the case for lower mass stars, for which some "non-standard" mixing processes have to be invoked to explain various chemical anomalies.

The physical ingredients of our models and numerical aspects of the computations are presented in Sects. 2 and 3 , respectively. The general properties of the stars and their chemical evolution during the phases prior to the TPAGB one are summarized in Sects. 4 and 5, respectively.

In Sect. 6, we briefly present the structural features of the thermal pulses and discuss the occurrence of the third dredge-up in our models. We derive a core-mass luminosity relation for our less massive stars that do not undergo hot-bottom burning. We then present the extrapolation procedure that we use to follow both the evolution and the nucleosynthesis in the "asymptotic regime" up to the TPAGB tip. We also give the initial mass-final mass relation resulting from our models, that appears to be in good agreement with the observed one.

In Sect. 7, we review the various nucleosynthesis sites inside TP-AGB stars, namely the burning shells $\left(\mathrm{He},{ }^{13} \mathrm{C}\right.$ and $\mathrm{H}$ ), the convective tongue that develops during a thermal runaway and the base of hot convective envelopes. We investigate in detail the nucleosynthetic processes that involve the $\mathrm{Li}, \mathrm{CNO}, \mathrm{F}, \mathrm{NeNa}$ and $\mathrm{MgAl}$ elements and neutrons. We then discuss which specific nuclear region, inside a TP-AGB star, mainly contributes to the surface abundance change of each nuclide when a third dredge-up occurs.

In Sect. 8, we finally present our predictions concerning (i) the evolution of all the surface isotopic ratios by including our evolutionary models and synthetic extrapolations up to the convective envelope removal and (ii) the chemical yields ejected at different ages for all the species up to ${ }^{28} \mathrm{Si}$. As in the previous sections, comparisons between our predictions and observations are attentively performed.

In this paper version, Sects. 2, 3, 5 and 8 have been somewhat reduced.

\section{Physics of the models}

\subsection{Structure}

\subsubsection{Equation of state}

Our equation of state is analytic. It includes

- the ionization of $\mathrm{H}, \mathrm{He}, \mathrm{C}, \mathrm{N}$ and $\mathrm{O}$. Complete ionization is assumed when OIX/OVIII > 50;

- the electrostatic corrections through the statistical model of Debye-Hückel for partially ionized regions and through the interpolation formalism of Koester (1976), between the Debye-Hückel and the ThomasFermi models, for the completely ionized matter;

- the electronic degeneracy as soon as its contribution to the total pressure exceeds $0.1 \%$;

- the production of neutrino pairs through four different processes, namely the photo-neutrinos, plasma neutrinos, $\left(\mathrm{e}^{+} \mathrm{e}^{-}\right)$pair annihilations and bremsstrahlung neutrinos. Energy loss contributions comes from Munakata et al. (1985), Itoh et al. (1992) and Kohyama et al. (1993).

\subsubsection{Opacities}

At low temperatures, i.e. below $8000 \mathrm{~K}$, we use the Alexander \& Ferguson (1994) opacity tables, very well suited for cool red giant envelopes and atmospheres.

At temperatures above $8000 \mathrm{~K}$, we use the OPAL opacity tables computed by Rogers \& Iglesias (1992). The abundance distribution of the heavy elements being scaled from the solar composition given by Anders \& Grevesse (1989) and updated by Grevesse (1991), as for low-temperature opacities.

For both sets of opacity tables, we first interpolate bilinearly in $T$ and $R=\log \left(\rho / T_{6}^{3}\right)$ for each table the composition of which is close to the model composition and then linearly in $X, Z$ (and $X(\mathrm{O})$ ) to obtain the right opacity coefficient corresponding to each shell. 
Finally, we used the Hubbard \& Lampe (1969) program to generate conductive opacity tables corresponding to the same chemical compositions and $(R, T)$ grid points as for the OPAL tables.

\subsubsection{Atmosphere treatment}

The stellar structure is integrated from the center to a very low optical depth $(\tau=0.001)$ in the atmosphere. We constraint the atmospheric temperature profile to correspond to those coming from realistic atmosphere models obtained by integrating the radiative transfer equation. More specifically, we use atmosphere models (1) from Plez (1992) for $T_{\text {eff }}<3900 \mathrm{~K}$, (2) from Eriksson (1994, private communication, using a physics similar to Bell et al. 1976) up to $5500 \mathrm{~K}$ and (3) computed with the Kurucz atmosphere program above $5500 \mathrm{~K}$. At each time step, we linearly interpolate a $T(\tau)$ profile corresponding to our model $T_{\text {eff }}, \log g$ and $Z$ from these grids of atmosphere models and then correspondingly modify the radiative pressure and gradient.

\subsubsection{Convection}

The structure of the convective regions is computed using the classical Mixing-Length Theory (MLT), as prescribed by Kippenhahn et al. (1968). Our models are standard in the sense that (1) the Schwarzschild criterion is considered to delimit the convective zones and (2) neither overshooting nor semi-convection have been considered in the present computations. The ratio $\alpha$ of the mixing-length free parameter over the pressure scale height has been put to a value of 1.5 , i.e. rather close to $\alpha \odot=1.64$ with which we fit the solar structure.

\subsubsection{Mass loss rates}

From the Main Sequence up to the thermally pulsing AGB phase, we used the empirical Reimers (1975) formula

$\dot{M}=-3.9810^{-13} \eta \frac{L R}{M} M_{\odot} \mathrm{yr}^{-1}$,

with $\eta=0.5$ up to the central He exhaustion.

From the beginning of the AGB phase, we adopted variable $\eta$ values, depending on the total mass of the star. More specifically, we took $\eta=2.5,3,3.5$ or 4 for our $3,4,5$ or $6 M_{\odot}$ models respectively, whatever $Z$. This $\eta$ increase with the total mass was found by Bryan et al. (1990) to give rather good results along the AGB. However, later during the thermally pulsing phase, Blöcker (1995) recently showed that the Bryan et al. (1990) formula underestimates the observed mass loss rate. This leads us to further increase $\eta$ (see Sect. 6.3). However, as mentioned by Vassiliadis \& Wood (1993), whatever empirical formula, the mass loss rate increase used in the models along the thermally pulsing AGB phase still remain uncertain by more than a factor of two. As demonstrated in Sect. 8, this important uncertainty on the mass loss rate formula conditions the AGB phase duration and consequently, the surface composition evolution through the successive third dredge-up events.

\subsubsection{Rate of gravitational energy change}

We note that along the thermally pulsing AGB phase, the thermal pulse features slightly depend on the way one estimates the rate $\varepsilon_{\text {grav }}$ of gravitational energy release or gain (see e.g. Kippenhahn \& Weigert 1991). So, let us specify that we wrote

$\varepsilon_{\text {grav }}=-\frac{D u}{D t}+\frac{P}{\rho^{2}} \frac{D \rho}{D t}$,

where $u$ is the specific (i.e. by unit mass) internal energy.

\subsection{Nucleosynthesis}

\subsubsection{Selected nuclides and network}

We follow the abundance evolution, through the whole stars, of 45 nuclides, namely the neutrons, all the 31 stable nuclides up to ${ }^{33} \mathrm{~S}$ as well as ${ }^{7} \mathrm{Be},{ }^{8} \mathrm{~B},{ }^{11} \mathrm{C},{ }^{14} \mathrm{C},{ }^{13} \mathrm{~N},{ }^{15} \mathrm{O}$, ${ }^{18} \mathrm{~F},{ }^{22} \mathrm{Na},{ }^{26} \mathrm{Al}$, ${ }^{26} \mathrm{Al}^{\mathrm{m}},{ }^{27} \mathrm{Si},{ }^{30} \mathrm{P}$ and ${ }^{31} \mathrm{~S}$.

These nuclides interact through a network containing 172 nuclear (neutron, proton and ${ }^{4} \mathrm{He}$ captures) and decay reactions. Many nuclear reaction rates are taken from Caughlan \& Fowler (1988). Let us just mention the nuclear reactions for which we selected another rate prescription.

$-{ }^{12} \mathrm{C}(\mathrm{n}, \gamma)$ from Nagai et al. (1991),

$-{ }^{12} \mathrm{C}(\alpha, \gamma)$ from Caughlan et al. (1985),

$-{ }^{13} \mathrm{C}(\mathrm{n}, \gamma)$ from Raman et al. (1990),

$-{ }^{14} \mathrm{C}(\mathrm{n}, \gamma)$ from Beer et al. (1991),

$-{ }^{14} \mathrm{C}(\mathrm{p}, \gamma)$ from Wiesher et al. (1990),

$-{ }^{14} \mathrm{C}(\alpha, \gamma)$ from Funck \& Langanke (1989),

$-{ }^{14} \mathrm{C}(\alpha, \mathrm{n}),{ }^{14} \mathrm{~N}(\mathrm{n}, \gamma),{ }^{15} \mathrm{~N}(\mathrm{n}, \gamma)$ and ${ }^{17} \mathrm{O}(\mathrm{n}, \alpha)$ from Fowler et al. (1967),

$-{ }^{14} \mathrm{~N}(\mathrm{n}, \mathrm{p})$ from Brehm et al. (1988),

$-{ }^{15} \mathrm{~N}(\alpha, \gamma)$ from De Oliveira (1995),

$-{ }^{16} \mathrm{O}(\mathrm{n}, \gamma)$ from Allen et al. (1971),

$-{ }^{17} \mathrm{O}(\mathrm{n}, \gamma)$ from Wagoner (1969),

$-{ }^{17} \mathrm{O}(\mathrm{p}, \gamma)$ and ${ }^{17} \mathrm{O}(\mathrm{p}, \alpha)$ from Landré et al. (1990),

$-{ }^{18} \mathrm{O}(\mathrm{n}, \gamma)$ from Thielemann (1991),

$-{ }^{19} \mathrm{~F}(\mathrm{n}, \gamma)$ and ${ }^{21} \mathrm{Ne}(\mathrm{n}, \gamma)$ from Bao \& Käppeler (1987),

$-{ }^{19} \mathrm{~F}(\mathrm{p}, \alpha)$ from Kious (1990),

$-{ }^{20} \mathrm{Ne}(\mathrm{n}, \gamma),{ }^{22} \mathrm{Ne}(\mathrm{n}, \gamma),{ }^{23} \mathrm{Na}(\mathrm{n}, \gamma), \quad{ }^{24} \mathrm{Mg}(\mathrm{n}, \gamma)$, ${ }^{25} \mathrm{Mg}(\mathrm{n}, \gamma)$ and ${ }^{26} \mathrm{Mg}(\mathrm{n}, \gamma)$ from Beer et al. (1991),

$-{ }^{22} \mathrm{Ne}(\alpha, \gamma)$ and ${ }^{22} \mathrm{Ne}(\alpha, \mathrm{n})$ from Hammer (1991),

$-{ }^{22} \mathrm{Na}(\mathrm{n}, \gamma),{ }^{23} \mathrm{Na}(\alpha, \mathrm{p}),{ }^{26} \mathrm{Al}^{\mathrm{g}}(\mathrm{n}, \gamma)$ and ${ }^{26} \mathrm{Al}^{\mathrm{m}}(\mathrm{n}, \gamma)$ from Woosley et al. (1978),

- ${ }^{24} \mathrm{Mg}(\alpha, \mathrm{p})$ and ${ }^{27} \mathrm{Al}(\mathrm{p}, \alpha)$ from Champagne et al. (1988),

- ${ }^{25} \mathrm{Mg}(\mathrm{p}, \gamma)$ and ${ }^{26} \mathrm{Mg}(\mathrm{p}, \gamma)$ from Illiadis (1990),

- and ${ }^{26} \mathrm{Al}^{\mathrm{g}}(\mathrm{p}, \gamma)$ and ${ }^{26} \mathrm{Al}^{\mathrm{g}}(\gamma){ }^{26} \mathrm{Al}^{\mathrm{m}}$ from Vogelaar (1989). 
On the other hand, the nuclear reaction rates for ${ }^{18} \mathrm{~F}(\mathrm{n}, \mathrm{p}),{ }^{18} \mathrm{~F}(\mathrm{n}, \alpha),{ }^{25} \mathrm{Mg}(\alpha, \gamma),{ }^{25} \mathrm{Mg}(\alpha, \mathrm{p})$, ${ }^{26} \mathrm{Mg}(\alpha, \gamma),{ }^{26} \mathrm{Mg}(\alpha, \mathrm{p}),{ }^{26} \mathrm{Mg}(\alpha, \mathrm{n}),{ }^{27} \mathrm{Al}(\alpha, \mathrm{n})$ as well as the 45 nuclear reactions involving $\mathrm{Si}, \mathrm{P}$ and $\mathrm{S}$ are fitted with a procedure developed by Rayet (1993, private communication). Let us finally mention recent improvements not yet incorporated in our network: various $(\mathrm{n}, \gamma)$ reactions rates on light nuclei (Nagai et al. 1995) and $\alpha$ capture rates on ${ }^{18} \mathrm{O}$ by Käppeler et al. (1994).

Finally, the nuclear screening factors are parameterized by using the Graboske et al. (1973) formalism, including weak, intermediate and strong screening cases.

\subsubsection{Neutron abundance}

Neutrons can be produced during the thermally pulsing AGB phase. They can, in particular, be captured by nuclides heavier than ${ }^{33} \mathrm{~S}$, mainly through $(\mathrm{n}, \gamma)$ reactions. As we do not follow the abundance of such elements, we created an additional nuclide, called ${ }^{34} \mathrm{~S}^{\Sigma}$, the mass fraction of which is set to

$X\left({ }^{34} \mathrm{~S}^{\Sigma}\right)=\sum_{a=34}^{209} X_{a}$.

This sum counts in fact 253 stable nuclides up to ${ }^{209} \mathrm{Bi}$. In order to have a good estimation of the free neutron abundance, we follow a method described by Jorissen \& Arnould (1989).

\subsubsection{Mixing inside convective zones}

If nuclear reactions occur inside a convective zone, most of them proceed in general with a longer time scale than the turn-over time scale associated to the motion of convective cells. In such a case, one usually consider the convective mixing as instantaneous. This allows to treat nucleosynthesis in one shot, by taking mass-weighted averages of the number abundances and nuclear reaction rates over the whole convective region.

However, if some key nuclides nuclearly evolve more rapidly than they are mixed, we have to consider the convection transport together with the nuclear reactions, so that nucleosynthesis equations become diffusion equations of the form

$\frac{\partial Y_{i}}{\partial t}=\frac{1}{\rho r^{2}} \frac{\partial}{\partial r}\left[\rho r^{2} D_{\mathrm{CONV}} \frac{\partial Y_{i}}{\partial r}\right]+$ nuclear terms,

where $Y_{i}=X_{i} / A_{i}$, the ratio of the mass fraction over the atomic mass of each nuclide $i$, and $D_{\mathrm{CONV}}=\bar{v}^{2} \tau_{\mathrm{CONV}}$, the convective diffusion coefficient, with $\bar{v}$ and $\tau_{\mathrm{CONV}}$ being the mean velocity and turn-over time of the convective cells, respectively. It is very $\mathrm{CPU}$ time consuming to treat the nucleosynthesis and convective mixing together through such diffusion equations for each nuclide in each convective shell for all the time steps. Consequently, as usual, instantaneous mixing has been assumed by default for our stellar evolution computations, except in two cases that potentially require a time-dependent treatment of the convective mixing: (1) the convective tongue associated with thermal pulses and (2) the base of the convective envelope when it is hot enough, both along the AGB phase.

We verified that inside thermal pulses, the elements that are the most affected by a diffusion treatment of the convective mixing are the very unstable nuclides (like ${ }^{22} \mathrm{Na}$ ), neutrons and protons. However, this do not change very significantly the nucleosynthesis global results (see however Sect. 7.5).

On the contrary, the mean turn-over time scale of the convective motions is much longer inside the convective envelope than inside a thermal pulse. If nuclear burning occurs at the base of the convective envelope, some important nuclear reactions can, depending on the bottom temperature, occur faster than the convective mixing so that a diffusion treatment is required. This is in particular the case for some of the reactions involved in the ${ }^{7} \mathrm{Li}$ synthesis (see Sect. 7.2).

\subsection{Initial models}

We have built initial models of $3,4,5,6$ and $7 M_{\odot}$ allowing us to start our computations at the beginning of the pre-main sequence phase (along the Hayashi track), when central temperatures are below $510^{5} \mathrm{~K}$.

For our $Z=0.005$ (0.02) models, the hydrogen mass fraction has been set to $X=0.745(0.687)$ and that of helium to $Y=0.250(0.293)$. The abundances of the heavier elements have been scaled on the Anders \& Grevesse (1989) abundance distribution.

\section{Numerical aspects}

We solve the five one-dimensional hydrodynamical equations for the evolution of the stellar structure with the mass coordinate as independent variable.

\subsection{Structure}

We first convert the five non-linear stellar structure differential equations into difference equations following the Henyey's scheme by defining discrete shells in mass. We then solve the resulting linearized system of equations by the Newton-Raphson relaxation procedure (see e.g. Press et al. 1986). Typically, 600 shells are required for a model on the main sequence phase, while along the AGB, 1000 to 1300 shells are needed (200 to 500 more shells being added during a thermal pulse).

On the other hand, typically 200 (700) models are necessary to model the central hydrogen (helium) burning phase. During the AGB phase, 2500 to 4500 models are required to model the time separating two successive thermal pulses, while the thermal pulse itself requires 100 to 350 models. 
Let us stress that these rather small numbers of mass shells and time steps have been chosen in order to save computer time and present a very large set of nucleosynthesis predictions. Straniero et al. (1996) very recently showed that this is probably in the disadvantage of the occurrence of the third dredge-up along the TP-AGB phase.

Finally, the mass $\Delta M=\dot{M} \Delta t$ lost during each time step $\Delta t$ is suppressed proportionally to the shell masses of the whole envelope above the burning regions.

\subsection{Nucleosynthesis}

The nucleosynthesis equations are solved using the Wagoner (1969) numerical technique, well suited for our purposes. However, if a diffusion treatment is required to simultaneously compute the time-dependent convective mixing and the nuclear burning, the diffusion Eqs. (4) are then solved with the same Newton-Raphson algorithm.

\section{Summary of the global evolution previous to TP-AGB phase}

\subsection{Some results}

Theoretical Hertzsprung-Russell diagrams (HRD) for the ensemble of our models are shown in Fig. 1, from the early PMS phase up to the beginning of the first thermal pulse (or He-shell flash) along the AGB phase.

The lifetimes are given in Table 1 for the PMS, the Hburning phase (from the zero-age-main sequence to central $\mathrm{H}$-exhaustion), the RGB phase (from central $\mathrm{H}$-exhaustion to central He-ignition), the He-burning phase (up to central He-exhaustion), the early (E) AGB phase (up to the first thermal pulse) and the thermally pulsing (TP) AGB phase. This last duration includes our extrapolation procedure up to the convective envelope exhaustion (see Sect. $6.3)$.

The occurrence of the first thermal pulse marks the end of the E-AGB phase and the beginning of the TP-AGB phase. This precise time, marked by the maximum surface luminosity just preceding the first major thermal instability, will be called $t_{0}$ in the following. Table 2 presents some characteristics of our models at $t_{0}$.

\subsection{Comparisons with other works}

For the main sequence duration, our results for $Z=0.02$ are in better agreement with Bressan et al. (1993) predictions than with the slightly higher values given by Schaller et al. (1992). The central helium burning lifetime crucially depends on the amount of overshooting. As our stellar models are computed without overshooting, we can only compare our predictions at this phase with those of the Padova group calculated in the same conditions; we obtain very similar results.

The other important quantity for the AGB phase is the core mass (i.e. the mass contained up to the HBS) at the end of the central He-burning phase. Indeed, its value determines the surface luminosity (through the core mass-luminosity relation; see e.g. Boothroyd \& Sackmann 1988a), the mass loss rate, and consequently the AGB phase duration. Last but not least, along the TP-AGB phase, the thermal pulses are stronger and the third dredge-up deeper when the core mass is higher. Our $3 M_{\odot}$ star with $Z=0.02$ enter the AGB phase with a core mass of $0.542 M_{\odot}$ (see Table 2 ), a value very close to that of the comparable model of Boothroyd \& Sackmann (1988a) or Lattanzio (1986), also computed without overshooting. Always for a reference $3 M_{\odot}$ star, the TP-AGB phase of the Straniero et al. (1995) models begins with a core mass of $0.53 M_{\odot}(2 \%$ lower $)$. At a comparable evolution stage however, the $5 M_{\odot}$ TP-AGB star of Vassiliadis \& Wood (1993), also computed without any kind of extra-mixing, has a core mass $\sim 4 \%$ higher than ours. This could be due to the somewhat lower metallicity of their models $(Z=0.016)$. In conclusion, the core mass at the beginning of the TP-AGB phase, although very important for this phase, still depends on various uncertainties related to the treatment of convection during the previous evolutionary phases.

\section{Surface abundances prior to TP-AGB phase}

\subsection{First and second dredge-up events}

During the first and second dredges-up, convective mixing and induced dilution modify the surface abundances which were unaltered until this phase (Iben 1964). Table 3 presents the resulting changes for the principal elements altered by H-burning. Let us emphasize the main points.

A second dredge-up occurs in our $\geq 4 M_{\odot}$ models, also leading to surface abundance changes. For our $\geq 5 M_{\odot}$ models, the convective envelope penetrates so deep that it pushes down the $\mathrm{H}-\mathrm{He}$ discontinuity.

\subsection{1. ${ }^{3} \mathrm{He}$}

${ }^{3} \mathrm{He}$ is produced at the beginning of the $\mathrm{T}$ Tauri phase from the initial ${ }^{2} \mathrm{H}$ burning. On the main sequence, a peak of ${ }^{3} \mathrm{He}$ appears, due to the competition of the reactions $\mathrm{p}\left(\mathrm{p}, \mathrm{e}^{+} \nu_{\mathrm{e}}\right){ }^{2} \mathrm{H}(\mathrm{p}, \gamma){ }^{3} \mathrm{He}$ and ${ }^{3} \mathrm{He}\left({ }^{3} \mathrm{He}, 2 \mathrm{p}\right){ }^{4} \mathrm{He}$. During the first dredge-up, the surface abundance of ${ }^{3} \mathrm{He}$ thus increases. However, the higher the stellar mass, the lower the ${ }^{3} \mathrm{He}$ peak, and the lower the surface enhancement of ${ }^{3} \mathrm{He}$ after the first dredge-up.

\subsubsection{CNO elements}

The first (and second) dredge-up phase(s) lead to a decrease of the ${ }^{12} \mathrm{C} /{ }^{13} \mathrm{C},{ }^{12} \mathrm{C} /{ }^{14} \mathrm{~N},{ }^{15} \mathrm{~N} /{ }^{14} \mathrm{~N}$ and ${ }^{16} \mathrm{O} /{ }^{17} \mathrm{O}$ isotopic ratios, the extent of which depends on both stellar mass and metallicity. 
Table 1. Lifetimes in all the evolutionary phases for the modeled stars which undergo thermal pulses

\begin{tabular}{lcccccccc}
\hline & \multicolumn{4}{c}{$Z=0.02$} & & \multicolumn{3}{c}{$Z=0.005$} \\
\cline { 2 - 5 } & $\overbrace{3 M_{\odot}}$ & $4 M_{\odot}$ & $5 M_{\odot}$ & $6 M_{\odot}$ & & $3 M_{\odot}$ & $4 M_{\odot}$ & $5 M_{\odot}$ \\
\hline \hline$\Delta t[\mathrm{PMS}](\mathrm{yr})$ & $1.2010^{7}$ & $5.8810^{6}$ & $3.7010^{6}$ & $2.1910^{6}$ & & $1.1010^{7}$ & $5.8210^{6}$ & $3.5010^{6}$ \\
$\Delta t[\mathrm{MS}](\mathrm{yr})$ & $2.8010^{8}$ & $1.3410^{8}$ & $7.6910^{7}$ & $5.0910^{7}$ & & $2.5910^{8}$ & $1.3010^{8}$ & $7.8810^{7}$ \\
$\Delta t[\mathrm{RG}](\mathrm{yr})$ & $1.7810^{7}$ & $6.3910^{6}$ & $2.8610^{6}$ & $1.2210^{6}$ & & $1.4510^{7}$ & $5.1710^{6}$ & $2.3810^{6}$ \\
$\Delta t[\mathrm{HB}](\mathrm{yr})$ & $1.5510^{8}$ & $5.2610^{7}$ & $2.6410^{7}$ & $1.0810^{7}$ & & $9.3710^{7}$ & $4.3010^{7}$ & $2.5910^{7}$ \\
$\Delta t[\mathrm{E}-\mathrm{AGB}](\mathrm{yr})$ & $6.1610^{6}$ & $2.8810^{6}$ & $1.2510^{6}$ & $5.9810^{5}$ & & $4.2910^{6}$ & $1.6910^{6}$ & $6.4110^{5}$ \\
$\Delta t[\mathrm{TP}-\mathrm{AGB}](\mathrm{yr})$ & $1.2210^{6}$ & $2.2610^{5}$ & $1.3310^{5}$ & $9.1210^{4}$ & & $9.0310^{5}$ & $1.5910^{5}$ & $7.9710^{4}$ \\
\hline
\end{tabular}

Table 2. Main features concerning the internal and surface structure of our models at the end of the E-AGB phase. [He] ([H]) specifies values taken at the maximum energy production rate in the helium (hydrogen) burning shell. $[\mathrm{H}-\mathrm{He}]$ refers to the inter-shell region (i.e. between the HeBS top and the HBS base) and [env] to the convective envelope bottom. $\Delta m$ is the mass thickness of a region

\begin{tabular}{|c|c|c|c|c|c|c|c|}
\hline & \multicolumn{4}{|c|}{$Z=0.02$} & \multicolumn{3}{|c|}{$Z=0.005$} \\
\hline & $3 M_{\odot}$ & $4 M_{\odot}$ & $5 M_{\odot}$ & $6 M_{\odot}$ & $3 M_{\odot}$ & $4 M_{\odot}$ & $5 M_{\odot}$ \\
\hline$t_{0}(\mathrm{yr})$ & $4.73910^{8}$ & $2.03410^{8}$ & $1.12310^{8}$ & $6.62410^{7}$ & $3.85110^{8}$ & $1.88010^{8}$ & $1.11710^{8}$ \\
\hline$L\left(L_{\odot}\right)$ & 3921 & 13274 & 19367 & 25743 & 5420 & 14761 & 21479 \\
\hline$R\left(R_{\odot}\right)$ & 182 & 398 & 486 & 554 & 182 & 339 & 387 \\
\hline$\Delta R[$ atmos. $]\left(R_{\odot}\right)$ & 3 & 15 & 17 & 18 & 3 & 10 & 10 \\
\hline$M\left(M_{\odot}\right)$ & 2.92 & 3.77 & 4.70 & 5.72 & 2.81 & 3.51 & 4.31 \\
\hline$\dot{M}\left(M_{\odot} \mathrm{yr}^{-1}\right)$ & $2.010^{-7}$ & $1.510^{-6}$ & $2.510^{-6}$ & $3.610^{-6}$ & $3.610^{-7}$ & $1.810^{-6}$ & $2.810^{-6}$ \\
\hline$T_{\text {eff }}(\mathrm{K})$ & 3381 & 3102 & 3088 & 3104 & 3667 & 3450 & 3550 \\
\hline $\log g_{\mathrm{eff}}\left(\mathrm{g} \mathrm{cm}^{-3}\right)$ & 0.38 & -0.19 & -0.26 & -0.29 & 0.36 & -0.08 & -0.10 \\
\hline$L_{\text {nucl }} / L$ & 0.96 & 0.87 & 0.80 & 0.71 & 0.94 & 0.79 & 0.66 \\
\hline$L_{\mathrm{He}} / L_{\mathrm{H}}$ & 0.09 & 0.08 & 0.07 & 0.06 & 0.06 & 0.13 & 0.10 \\
\hline$\eta_{\mathrm{c}}$ & 20 & 30 & 37 & 39 & 20 & 32 & 39 \\
\hline$T_{\max }(\mathrm{K})$ & $2.1110^{8}$ & $3.4810^{8}$ & $3.9710^{8}$ & $4.6010^{8}$ & $2.4410^{8}$ & $3.9110^{8}$ & $4.5410^{8}$ \\
\hline$m_{T \max }\left(M_{\odot}\right)$ & 0.320 & 0.521 & 0.643 & 0.745 & 0.373 & 0.608 & 0.736 \\
\hline$r[\mathrm{He}]\left(R_{\odot}\right)$ & 0.00008 & 0.00003 & 0.00003 & 0.00002 & 0.00008 & 0.00004 & 0.00003 \\
\hline$m[\mathrm{He}]\left(M_{\odot}\right)$ & 0.520 & 0.732 & 0.815 & 0.894 & 0.594 & 0.814 & 0.903 \\
\hline$\Delta m[\mathrm{He}]\left(M_{\odot}\right)$ & 0.08 & 0.03 & 0.02 & 0.24 & 0.04 & 0.01 & 0.23 \\
\hline$T[\mathrm{He}](\mathrm{K})$ & $1.4310^{8}$ & $1.6110^{8}$ & $1.6410^{8}$ & $1.6810^{8}$ & $1.3710^{8}$ & $1.6410^{8}$ & $1.6810^{8}$ \\
\hline$\rho[\mathrm{He}]\left(\mathrm{g} \mathrm{cm}^{-3}\right)$ & 13.8 & 7.7 & 5.9 & 5.9 & 11.2 & 5.3 & 4.6 \\
\hline$\Delta m[\mathrm{H}-\mathrm{He}]\left(M_{\odot}\right)$ & $4.010^{-3}$ & $4.710^{-4}$ & $1.510^{-4}$ & $6.010^{-5}$ & $3.310^{-3}$ & $3.810^{-4}$ & $1.210^{-4}$ \\
\hline$r[\mathrm{H}]\left(R_{\odot}\right)$ & 0.00016 & 0.00006 & 0.00004 & 0.00003 & 0.00015 & 0.00006 & 0.00005 \\
\hline$m[\mathrm{H}]\left(M_{\odot}\right)$ & 0.542 & 0.738 & 0.818 & 0.895 & 0.609 & 0.818 & 0.905 \\
\hline$\Delta m[\mathrm{H}]\left(M_{\odot}\right)$ & $1.210^{-3}$ & $3.210^{-4}$ & $1.810^{-4}$ & $5.510^{-3}$ & $9.710^{-4}$ & $3.410^{-4}$ & $2.210^{-4}$ \\
\hline$T[\mathrm{H}](\mathrm{K})$ & $5.0710^{7}$ & $6.4310^{7}$ & $7.1010^{7}$ & $7.7710^{7}$ & $5.7510^{7}$ & $7.0010^{7}$ & $7.7410^{7}$ \\
\hline$\rho[\mathrm{H}]\left(\mathrm{g} \mathrm{cm}^{-3}\right)$ & $2.010^{-2}$ & $1.410^{-2}$ & $1.310^{-2}$ & $1.110^{-2}$ & $2.410^{-2}$ & $1.710^{-2}$ & $1.610^{-2}$ \\
\hline$r[\mathrm{env}]\left(R_{\odot}\right)$ & 0.0042 & 0.0008 & 0.0002 & 0.0001 & 0.0044 & 0.0008 & 0.0002 \\
\hline$m[\mathrm{env}]\left(M_{\odot}\right)$ & 0.544 & 0.738 & 0.818 & 0.896 & 0.612 & 0.819 & 0.905 \\
\hline$T[\mathrm{env}](\mathrm{K})$ & $2.6810^{6}$ & $5.7210^{6}$ & $1.5810^{7}$ & $4.2710^{7}$ & $2.7110^{6}$ & $6.5310^{6}$ & $2.2510^{7}$ \\
\hline$\rho[\mathrm{env}]\left(\mathrm{g} \mathrm{cm}^{-3}\right)$ & $1.110^{-3}$ & $4.110^{-3}$ & $5.710^{-2}$ & 0.9 & $1.010^{-3}$ & $6.010^{-3}$ & 0.2 \\
\hline
\end{tabular}




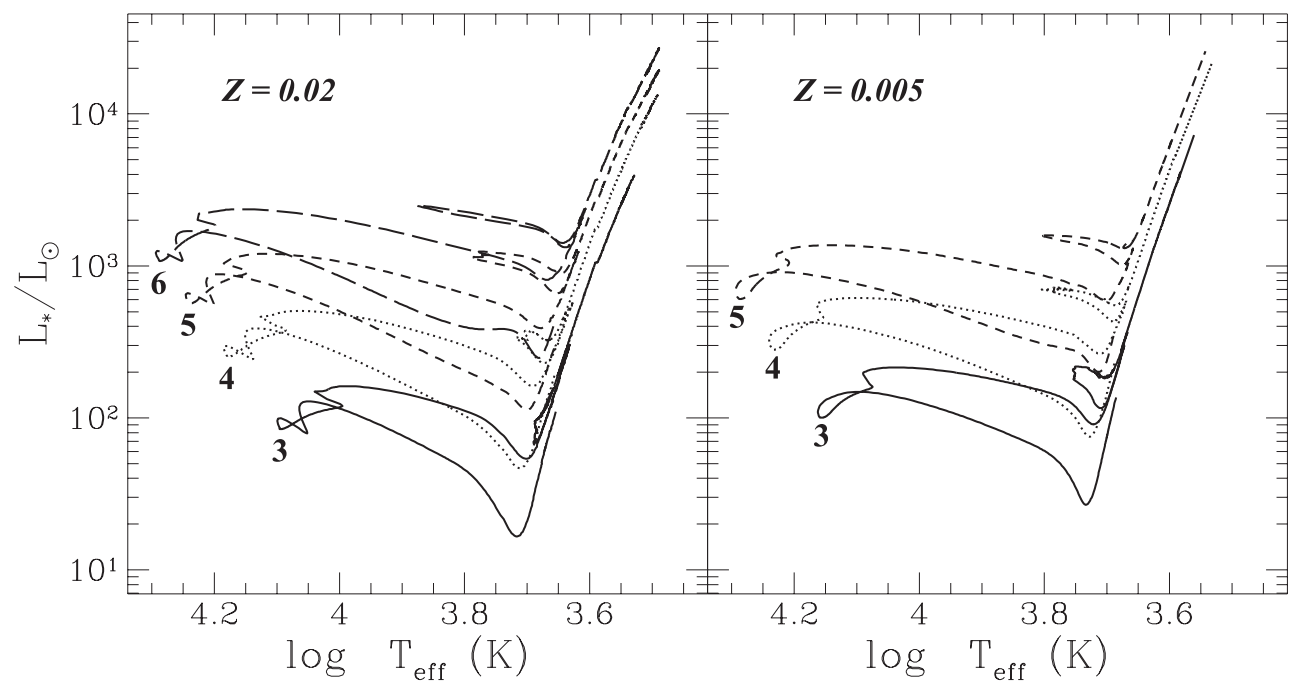

Fig. 1. Theoretical HRD, from the beginning of the PMS phase up to the beginning of the first thermal pulse along the AGB phase, for $Z=0.02$ (left) and $Z=0.005$ (right)

Table 3. Surface abundances and isotopic ratios consecutive to the first and second dredge-up episodes (i.e. respectively at the top RG and time $t_{0}$ defined in the text)

\begin{tabular}{|c|c|c|c|c|c|c|c|c|}
\hline & & & & 0.02 & & & $Z=0.005$ & \\
\hline & & $3 M_{\odot}$ & $4 M_{\odot}$ & $5 M_{\odot}$ & $6 M_{\odot}$ & $3 M_{\odot}$ & $4 M_{\odot}$ & $5 M_{\odot}$ \\
\hline${ }^{3} \mathrm{He}$ & $\begin{array}{l}\text { initial } \\
\text { top } R G \\
\text { at } t_{0}\end{array}$ & $\begin{array}{l}3.2210^{-5} \\
1.5510^{-4} \\
1.5410^{-4}\end{array}$ & $\begin{array}{l}3.1910^{-5} \\
1.0510^{-4} \\
1.0310^{-4}\end{array}$ & $\begin{array}{l}3.1310^{-5} \\
8.5210^{-5} \\
7.7910^{-5}\end{array}$ & $\begin{array}{l}3.1210^{-5} \\
7.3210^{-5} \\
6.6510^{-5}\end{array}$ & $\begin{array}{l}2.7110^{-5} \\
1.7110^{-4} \\
1.6610^{-4}\end{array}$ & $\begin{array}{l}2.7110^{-5} \\
1.2810^{-4} \\
1.1310^{-4}\end{array}$ & $\begin{array}{l}2.6610^{-5} \\
1.0810^{-4} \\
8.5610^{-5}\end{array}$ \\
\hline${ }^{4} \mathrm{He}$ & $\begin{array}{l}\text { initial } \\
\text { top } R G \\
\text { at } t_{0}\end{array}$ & $\begin{array}{l}0.2932 \\
0.3047 \\
0.3054\end{array}$ & $\begin{array}{l}0.2932 \\
0.3039 \\
0.3052\end{array}$ & $\begin{array}{l}0.2932 \\
0.2995 \\
0.3367\end{array}$ & $\begin{array}{l}0.2932 \\
0.3017 \\
0.3421\end{array}$ & $\begin{array}{l}0.2495 \\
0.2585 \\
0.2624\end{array}$ & $\begin{array}{l}0.2495 \\
0.2505 \\
0.2629\end{array}$ & $\begin{array}{l}0.2495 \\
0.2498 \\
0.3014\end{array}$ \\
\hline${ }^{7} \mathrm{Li}$ & $\begin{array}{l}\text { initial } \\
\text { top } R G \\
\text { at } t_{0}\end{array}$ & $\begin{array}{c}9.9010^{-9} \\
1.4410^{-10} \\
1.2010^{-10}\end{array}$ & $\begin{array}{c}9.9010^{-9} \\
1.4410^{-10} \\
9.8510^{-11}\end{array}$ & $\begin{array}{c}9.9010^{-9} \\
1.4310^{-10} \\
7.3910^{-11}\end{array}$ & $\begin{array}{c}9.9010^{-9} \\
1.4410^{-10} \\
7.5310^{-17}\end{array}$ & $\begin{array}{c}2.4710^{-9} \\
3.1410^{-11} \\
1.4910^{-11}\end{array}$ & $\begin{array}{c}2.4710^{-9} \\
3.5110^{-11} \\
1.6810^{-11}\end{array}$ & $\begin{array}{c}2.4710^{-9} \\
3.8610^{-11} \\
3.5910^{-16}\end{array}$ \\
\hline${ }^{12} \mathrm{C} /{ }^{13} \mathrm{C}$ & $\begin{array}{l}\text { initial } \\
\text { top } R G \\
\text { at } t_{0}\end{array}$ & $\begin{array}{l}83.1 \\
19.3 \\
19.1\end{array}$ & $\begin{array}{l}83.1 \\
19.0 \\
18.6\end{array}$ & $\begin{array}{l}83.1 \\
18.8 \\
17.8\end{array}$ & $\begin{array}{l}83.1 \\
18.5 \\
17.5\end{array}$ & $\begin{array}{l}83.1 \\
18.7 \\
18.1\end{array}$ & $\begin{array}{l}83.1 \\
18.4 \\
17.5\end{array}$ & $\begin{array}{l}83.1 \\
18.3 \\
17.4\end{array}$ \\
\hline${ }^{12} \mathrm{C} /{ }^{14} \mathrm{~N}$ & $\begin{array}{l}\text { initial } \\
\text { top } R G \\
\text { at } t_{0}\end{array}$ & $\begin{array}{l}2.745 \\
0.725 \\
0.712\end{array}$ & $\begin{array}{l}2.745 \\
0.714 \\
0.684\end{array}$ & $\begin{array}{l}2.745 \\
0.769 \\
0.565\end{array}$ & $\begin{array}{l}2.745 \\
0.735 \\
0.538\end{array}$ & $\begin{array}{l}2.745 \\
0.636 \\
0.581\end{array}$ & $\begin{array}{l}2.745 \\
0.888 \\
0.610\end{array}$ & $\begin{array}{l}2.745 \\
1.100 \\
0.496\end{array}$ \\
\hline${ }^{14} \mathrm{~N} /{ }^{15} \mathrm{~N}$ & $\begin{array}{l}\text { initial } \\
\text { top } R G \\
\text { at } t_{0}\end{array}$ & $\begin{array}{c}253 \\
1243 \\
1270\end{array}$ & $\begin{array}{c}253 \\
1281 \\
1346\end{array}$ & $\begin{array}{c}253 \\
1210 \\
1654\end{array}$ & $\begin{array}{c}253 \\
1279 \\
1774\end{array}$ & $\begin{array}{c}253 \\
1397 \\
1546\end{array}$ & $\begin{array}{c}253 \\
1033 \\
1510\end{array}$ & $\begin{array}{c}253 \\
856 \\
1841\end{array}$ \\
\hline${ }^{12} \mathrm{C} /{ }^{16} \mathrm{O}$ & $\begin{array}{l}\text { initial } \\
\text { top } R G \\
\text { at } t_{0}\end{array}$ & $\begin{array}{l}0.316 \\
0.205 \\
0.204\end{array}$ & $\begin{array}{l}0.316 \\
0.207 \\
0.205\end{array}$ & $\begin{array}{l}0.316 \\
0.210 \\
0.206\end{array}$ & $\begin{array}{l}0.316 \\
0.211 \\
0.207\end{array}$ & $\begin{array}{l}0.316 \\
0.194 \\
0.191\end{array}$ & $\begin{array}{l}0.316 \\
0.208 \\
0.192\end{array}$ & $\begin{array}{l}0.316 \\
0.229 \\
0.197\end{array}$ \\
\hline${ }^{16} \mathrm{O} /{ }^{17} \mathrm{O}$ & $\begin{array}{l}\text { initial } \\
\text { top } R G \\
\text { at } t_{0}\end{array}$ & $\begin{array}{c}2467 \\
314 \\
311\end{array}$ & $\begin{array}{c}2467 \\
409 \\
391\end{array}$ & $\begin{array}{c}2467 \\
562 \\
508\end{array}$ & $\begin{array}{c}2467 \\
617 \\
579\end{array}$ & $\begin{array}{c}2467 \\
258 \\
245\end{array}$ & $\begin{array}{c}2467 \\
869 \\
323\end{array}$ & $\begin{array}{c}2467 \\
1814 \\
358\end{array}$ \\
\hline${ }^{16} \mathrm{O} /{ }^{18} \mathrm{O}$ & $\begin{array}{l}\text { initial } \\
\text { top } R G \\
\text { at } t_{0}\end{array}$ & $\begin{array}{l}442 \\
589 \\
591\end{array}$ & $\begin{array}{l}442 \\
582 \\
588\end{array}$ & $\begin{array}{l}442 \\
577 \\
588\end{array}$ & $\begin{array}{l}442 \\
577 \\
588\end{array}$ & $\begin{array}{l}442 \\
616 \\
623\end{array}$ & $\begin{array}{l}442 \\
573 \\
619\end{array}$ & $\begin{array}{l}442 \\
522 \\
611\end{array}$ \\
\hline
\end{tabular}


- From an initial value equal to 83 , the ${ }^{12} \mathrm{C} /{ }^{13} \mathrm{C}$ ratio decreases to $\simeq 18-19$. The higher the stellar mass, the lower the final carbon isotopic ratio.

This ratio slightly further decreases after the second dredge-up.

- For $Z=0.02$, the maximum depth of the convective envelope reaches the deepest step of the ${ }^{14} \mathrm{~N}$ profile, for $\geq 3 M_{\odot}$ stars. This implies that ${ }^{12} \mathrm{C} /{ }^{14} \mathrm{~N}$, as well as ${ }^{15} \mathrm{~N} /{ }^{14} \mathrm{~N}$ have almost the same value in these models after the first dredge-up. On the contrary, for the $Z=0.005$ objects, the lower the stellar mass, the less efficient the dredge-up, and consequently, the less ${ }^{14} \mathrm{~N}$ is up-heaved to the surface. This explains the trend for the final ${ }^{12} \mathrm{C} /{ }^{14} \mathrm{~N}$ ratio versus stellar mass (see Charbonnel 1994 for more details).

After the second dredge-up, whatever $Z$, the convective envelope of $\geq 4 M_{\odot}$ stars penetrates deeper for increasing stellar mass as attested by both ${ }^{12} \mathrm{C} /{ }^{14} \mathrm{~N}$ and ${ }^{15} \mathrm{~N} /{ }^{14} \mathrm{~N}$ ratios.

- The theoretical post dredge-up value of ${ }^{16} \mathrm{O} /{ }^{17} \mathrm{O}$ increases with the stellar mass. Moreover, since for $Z=$ 0.005 the deepening convective envelope of our 4 and $5 M_{\odot}$ stars does not reach the regions where the $\mathrm{ON}$ cycle operated, the post dredge-up values of ${ }^{16} \mathrm{O} /{ }^{17} \mathrm{O}$ are higher than for $Z=0.02$.

After the second dredge-up however, ${ }^{17} \mathrm{O}$ enriched matter is up-heaved in the convective envelope leading to very low ${ }^{16} \mathrm{O} /{ }^{17} \mathrm{O}$.

- The predicted post dredge-up value of ${ }^{16} \mathrm{O} /{ }^{18} \mathrm{O}$ slightly decreases with increasing stellar mass.

\subsection{Comparisons with other works}

Our ${ }^{3} \mathrm{He}$ surface abundance increases are in rather good agreement with the predictions by Bressan et al. (1993).

Concerning the CNO elements, our results are in general good agreement with the predictions by Schaller et al. (1992), Bressan et al. (1993) and El Eid (1994). The only important discrepancy between theoretical predictions of the different groups for intermediate-mass stars concerns the post dredge-up ${ }^{16} \mathrm{O} /{ }^{17} \mathrm{O}$ ratio, which value highly depends on the adopted ${ }^{17} \mathrm{O}(\mathrm{p}, \alpha){ }^{14} \mathrm{~N}$ and ${ }^{17} \mathrm{O}(\mathrm{p}, \gamma){ }^{18} \mathrm{~F}$ reaction rates. We use the rates given by Landré et al. (1990), as do Schaller et al. (1992), and we obtain ${ }^{16} \mathrm{O} /{ }^{17} \mathrm{O}$ ratios very similar to those of the Geneva group. On the other hand, Bressan et al. (1993) use the lower ${ }^{17} \mathrm{O}$ proton capture rates given by Caughlan \& Fowler (1988), and obtain much lower ${ }^{16} \mathrm{O} /{ }^{17} \mathrm{O}$ ratios (see also El Eid (1994) for a discussion of the influence of the adopted ${ }^{17} \mathrm{O}$ destruction rate on the resulting ${ }^{16} \mathrm{O} /{ }^{17} \mathrm{O}$ ratio).

\subsection{Comparisons with observations}

In our intermediate-mass stars, the theoretical post dredge-up values of the carbon isotopic ratio are slightly lower than the observations by Gilroy (1989) in galactic cluster giants.

Red giants in this evolutionary phase present ${ }^{16} \mathrm{O} /{ }^{17} \mathrm{O}$ ratios between 300 to 1000 and ${ }^{16} \mathrm{O} /{ }^{18} \mathrm{O}$ ratios in the range 400 to 600 (Harris et al. 1988; Smith \& Lambert 1990a). This is in very good agreement with our predictions. In particular, our prediction is in perfect agreement with the observed value in $\alpha$ UMa (Harris et al. 1988), which estimated mass is roughly $3 M_{\odot}$. This point is in favor of high ${ }^{17} \mathrm{O}$ destruction rates.

This agreement between standard models and observations confirms that no extra-mixing (diffusion, rotationinduced mixing, ... ) is expected to occur in intermediatemass stars, as already discussed in Charbonnel (1994, 1995).

\subsection{Internet tables}

Very complete tables containing information about the center and surface evolution of the structure and chemical composition for our $3,4,5,6$ and $7 M_{\odot}$ models with $Z=0.005$ and 0.02 , from the beginning of the PMS phase up to the end of the E-AGB phase, can be found through the internet network at the following address and path:

http://www-laog.obs.ujf-grenoble.fr/liens/starevol/evol.html

More specifically, we present, for each star, the evolution of

- the age, mass ,mass loss rate, surface luminosity, bolometric magnitude, effective temperature, total radius, surface density and gravity;

- the central temperature, density, pressure, degeneracy parameter and the mass fraction of ${ }^{1} \mathrm{H},{ }^{4} \mathrm{He},{ }^{12} \mathrm{C}$ and ${ }^{16} \mathrm{O}$;

- the mass and radius positions of the central convective core boundary and of the bottom of the convective envelope as well as its temperature and density;

- the nuclear luminosities of the $\mathrm{H}$ - and He-burning shells;

- the mass and radius positions of the $\mathrm{H}$ - and He-burning shells;

- the surface mass fractions of ${ }^{1} \mathrm{H},{ }^{3} \mathrm{He},{ }^{4} \mathrm{He},{ }^{7} \mathrm{Li},{ }^{9} \mathrm{Be}$, ${ }^{12} \mathrm{C},{ }^{13} \mathrm{C},{ }^{14} \mathrm{~N},{ }^{15} \mathrm{~N},{ }^{16} \mathrm{O},{ }^{17} \mathrm{O},{ }^{18} \mathrm{O},{ }^{19} \mathrm{~F},{ }^{20} \mathrm{Ne},{ }^{21} \mathrm{Ne},{ }^{22} \mathrm{Ne}$, ${ }^{23} \mathrm{Na},{ }^{24} \mathrm{Mg},{ }^{25} \mathrm{Mg},{ }^{26} \mathrm{Mg},{ }^{26} \mathrm{Al}^{\mathrm{g}},{ }^{27} \mathrm{Al}$.

\section{Structural properties of the thermal pulses}

\subsection{Evolutionary properties of the thermal pulses as a function of $M$ and $Z$}

As the stars are ascending the AGB, the features of the thermal pulses evolve, and some of the main characterizing quantities of these events reach "asymptotic values". The most important feature is the thermal pulse intensity that can be defined in terms of the HeBS luminosity $L_{\mathrm{He}}^{\max }$ reached at the maximum of the He-shell 
flash. $L_{\mathrm{He}}^{\max }$ is quite low during the first thermal pulses, but it rapidly increases with core mass (or flash number). The increasing rate is greater for lower metallicities or higher stellar masses. After typically 10 (5) thermal pulses in our $Z=0.02(0.005)$ models, the thermal pulse intensity is very high and only slightly increases from pulse to pulse, monotonously with the core mass. This second part of the TP-AGB phase is usually called the "asymptotic regime" or "full amplitude" of the thermal pulses.

For each of the modeled stars, we have computed at least four full amplitude thermal pulses. Let us briefly discuss the main evolutionary properties of these thermal runaways.

The thermal runaways are stronger inside stars of lower $Z$, which facilitates dredge-up events (see Sect. 6.2). The intensities of our He-shell flashes are comparable to other published values for stars of about the same core masses (see e.g. Boothroyd \& Sackmann 1988b and Table 4).

While the stars evolve along the TP-AGB, the HeBS and HBS advance in mass, and the mean inter-shell mass slightly decreases with time. From one pulse to another, the top of the convective tongue at its maximum extension gets closer from the HBS, even if contact between both regions has never been found due to the fact that the HBS is still active at that time. Moreover, for all the thermal runaways we have computed, the overlap in mass between two successive thermal pulses is ranged between 0.6 and 0.4 for AGB stars of increasing total mass. This overlap is slightly larger for our lower $Z$ models.

The maximum (minimum) temperature $T_{\mathrm{b}}^{\max }\left(T_{\mathrm{t}}^{\min }\right)$ and density $\rho_{\mathrm{b}}^{\max }\left(\rho_{\mathrm{t}}^{\min }\right)$ reached at the base (top) of a thermal pulse both increase (decrease) from pulse to pulse. For a given He-shell flash, $T_{\mathrm{b}}^{\max }$ increases with stellar mass and metallicity, while $\rho_{\mathrm{b}}^{\max }$ decreases with stellar mass but increases with metallicity. In the asymptotic regime, the growth rate of $T_{\mathrm{b}}^{\max }$ also tends to an asymptotic value. By comparison with other works for the $3 M_{\odot}(Z=0.02)$ star, we found that our $T_{\mathrm{b}}^{\max }$ are typically $4 \%$ higher than those of Boothroyd \& Sackmann (1988b) and Straniero et al. (1996). This could be mainly due to (i) different prescriptions to compute $\varepsilon_{\text {grav }}$, (ii) different envelope masses due to different mass loss rates and (iii) different MLT treatments (see Sect. 2.1.4). The temperature and density differences between the base and top of each convective tongue are rather large. This point is important for nucleosynthesis purposes (see Sect. 7).

The duration of the convective tongue associated with each thermal pulse $\left(\Delta t_{\mathrm{p}}\right)$ and, to a lower extent, that of the inter-pulse phase $\left(\Delta t_{\text {ip }}\right)$, are crucial quantities for the nucleosynthesis too. The stronger a He-flash, the shorter it is and the quickest it deactivates. Both quantities are thus smaller for higher core masses and lower metallicities. Moreover, $\Delta t_{\mathrm{p}}$ tends to decrease as the stars evolve along the TP-AGB. $\Delta t_{\text {ip }}$ also decreases after the first He-shell flashes, but it then reaches an almost constant value dur- ing the asymptotic regime, only very slightly decreasing with the increasing core mass.

\subsection{Third dredge-up}

Along the TP-AGB phase, the bottom of the convective envelope can penetrate deep inside regions that have been nuclearly processed just after a thermal pulse, if it is strong enough (in order to give rise to a large enough entropy increase in the inter-shell region). Contrarily to the first and second dredges-up, this so-called third dredge-up, when it occurs, successively mixes up to the surface (i) material that has experienced H-burning in the thin HBS but also (ii) part of the region where the thermal pulse nucleosynthesis operated just before. Both regions give rise to very different - and sometimes opposite - chemical pollutions of the convective envelope (see Sects. 7.1 and 7.6).

In our $Z=0.02$ models, the HBS region is already dredged-up from the 14th, 5th and 3rd thermal pulse for the 3,4 and $5 M_{\odot}$ stars, respectively, while for the $Z=0.005$ models, such a mixing already occurs from the 2nd and 1st thermal pulse for the 3 and $4 M_{\odot}$ stars, respectively. The $6 M_{\odot}\left(5 M_{\odot}\right)$ star with $Z=0.02(0.005)$ continuously mixes H-burning products up to the surface as it experiences H-burning at the bottom of its convective envelope (the so-called "Hot-Bottom Burning" or HBB; see Sect. 7.1). This kind of dredge-up already modifies the surface composition for some species $\left(\right.$ like ${ }^{26} \mathrm{Al}^{\mathrm{g}}$; see Sects. 7.4 and 7.6).

The complete third dredge-up (one usually simply calls the third dredge-up, 3DUP hereafter), i.e. reaching the upper part of the previous thermal pulse region, is more difficult to obtain numerically. Following models (Wood 1981; Iben 1983; Lattanzio 1987; Boothroyd \& Sackmann 1988c), the dredge-up of thermal pulse material is easier for stars of lower metallicity $Z$. This is supported by observations (see e.g. Blanco et al. 1978). In our models, we just found a 3DUP from the 9th thermal pulse of the $4 M_{\odot}$ with $Z=0.005$ star. However, based on the evolution of the post-thermal pulse entropy profile from pulse to pulse, we can predict that such a dredgeup will also occur, within the next two thermal pulses, for our 5 and $6 M_{\odot}$ stars with $Z=0.02$ and for the $5 M_{\odot}$ star with $Z=0.005$ (see Table 4 for the number of computed thermal pulses for each object). Some additional thermal pulses are still needed for lower mass objects. Several years ago, the 3DUP was found to occur too late along the TP-AGB phase for AGB stars of decreasing mass and/or increasing metallicity (see e.g. Iben 1976; Fujimoto \& Sugimoto 1979). This was a common problem (see Lattanzio 1989 for a review). Recent works showed that with the new OPAL opacities, the 3DUP can now be obtained even for low-mass Pop I objects (see e.g. Frost \& Lattanzio 1996 or Straniero et al. 1995).

One of the clearest signature of the 3DUP is the significant ${ }^{12} \mathrm{C}$ pollution of the convective envelope, brought into 
the inter-shell region by the convective tongue of a thermal pulse (see Sect. 8.1). As the thermal pulse intensity (and consequently the probability of 3DUP occurrence) increases with time, as do the core mass and the total luminosity, the models tend predict the formation of carbon (C) stars (for which the surface ${ }^{12} \mathrm{C} /{ }^{16} \mathrm{O}$ becomes greater than unity thanks to the repetitive 3DUP events) at too high luminosities, i.e. to late during the TP-AGB phase, especially for solar metallicity stars. Solutions to increase the depth of the convective envelope during a dredgeup, e.g. by treating in some way semi-convection (see e.g. Lattanzio 1986) or by invoking undershooting (see Alongi et al. 1991) have been suggested. They indeed help the 3DUP to occur but do not yet solve completely the problem. Fundamentally, this could be due to our bad knowledge of convection inside stars. Very recently, Straniero et al. (1996) suggested that by considerably increasing the number of mass shells and especially the number of time steps to model thermal pulses, the 3DUP occurs earlier during the TP-AGB phase. This consists in a real numerical progress in that field. The fact we find 3DUP latter than them along the AGB phase can be due to our lower number of time steps (typically by a factor of 3 ).

\subsection{Towards the end of TP-AGB phase}

\subsubsection{Core mass-luminosity relation}

It was first discovered by Paczyński (1975) that during the asymptotic TP-AGB phase, there is a linear relation between the surface luminosity $L$ and the core mass $M_{\mathrm{C}} \equiv m[\mathrm{H}]$ (see Table 2) which is only slightly dependent on the total mass of the AGB star. Blöcker \& Schönberner (1991) however found that this kind of relation does not exist for the most massive AGB stars for which the bottom of the convective envelope goes down to the HBS so that part of the H-burning occurs inside the convective envelope (HBB).

From our models for the last four thermal pulses of the 3 and $4 M_{\odot}$ with $Z=0.02$ and the $3 M_{\odot}$ with $Z=0.005$, we have derived a core mass-luminosity relation in the form

$$
\begin{aligned}
\frac{L}{L_{\odot}}= & \alpha\left(\frac{M}{M_{\odot}}\right)^{\beta}\left(\frac{Z}{0.02}\right)^{\gamma}\left[\frac{M_{\mathrm{C}}}{M_{\odot}}-\right. \\
& \left.\delta\left(\frac{M}{M_{\odot}}\right)^{\varepsilon}\left(\frac{Z}{0.02}\right)^{\zeta}\right],
\end{aligned}
$$

where $\alpha=1233 L_{\odot}, \beta=3.566, \gamma=0.519, \delta=0.1057$, $\varepsilon=1.312$ and $\zeta=0.203$. It is in good agreement with other $M_{\mathrm{C}}-L$ relations, for $0.50<M_{\mathrm{C}} / M_{\odot}<1.10$ and $0.005 \leq Z \leq 0.02$ (see e.g. Lattanzio 1986 or Boothroyd $\&$ Sackmann 1988a). The more massive stars experience HBB and do not fit, consequently, to the same relation. In Table 4, we however give a linear relation of the form
$L / L_{\odot}=\alpha\left(M_{\mathrm{C}} / M_{\odot}-\beta\right)$ for the $5 M_{\odot}\left(4 M_{\odot}\right)$ AGB star with $Z=0.02(0.005)$ that do not experience a very strong HBB. Let us stress that during the TP-AGB phase, $L$ suffers very important variations during and after each thermal pulse. Frequently, the core mass-luminosity relation is derived by taking solely account of the maximum surface luminosity preceding each thermal pulse. However, the probability to observe an AGB star just at that time is very weak $\left(\sim \Delta t_{\mathrm{p}} / \Delta t_{\mathrm{ip}}<1 \%\right.$; see Table 4$)$. We thus preferred to derive our relations with a more realistic surface luminosity that is averaged over each pulse-inter-pulse cycle.

On the other hand, it is well known that the rate of mass advance of a burning shell (here $M_{\mathrm{C}}$ for the HBS) is related to the rate of nuclear energy production by

$\frac{\mathrm{d} M_{\mathrm{C}}}{\mathrm{d} t} \simeq \frac{L_{\mathrm{H}}}{\bar{X} E_{\mathrm{H}}}$

with $L_{\mathrm{H}}$ being the luminosity produced by the HBS, $\bar{X}$ the mean hydrogen mass fraction over this burning shell and $E_{\mathrm{H}}=6.3510^{18} \mathrm{erg} \mathrm{g}^{-1}$ the amount of energy produced by the conversion of $1 \mathrm{~g}$ of ${ }^{1} \mathrm{H}$ to ${ }^{4} \mathrm{He}$ through the CNO bi-cycle. Along the TP-AGB, most of the surface luminosity $L$ is produced by the HBS. As indicated in Table 2, the HeBS contributes much less than the HBS to the nuclear luminosity (except during a thermal runaway). Gravitation also constitutes a relatively small energy source, especially for the less massive AGB stars. In conclusion, we can write $L_{\mathrm{H}} \simeq \xi L$ with $0.6<\xi<0.9$ depending on $M$. By eliminating $L_{\mathrm{H}}$ in Eq. (6) with the core mass-luminosity relation, one finds that the core mass $M_{\mathrm{C}}$ increases exponentially with time during the asymptotic TP-AGB phase. The time scale for the core mass growth is $\sim 10^{6}\left(\sim 10^{5}\right)$ yr for a $3 M_{\odot}\left(6 M_{\odot}\right)$ AGB star, that is, interestingly, comparable to the total duration of the TP-AGB phase. Table 5 demonstrates that Eq. (6) predicts rates of HBS advance in mass that are very similar to those coming from our numerical models, except for the $6 M_{\odot}\left(5 M_{\odot}\right)$ object with $Z=0.02(0.005)$, probably due to the strong HBB.

\subsubsection{Extrapolation procedure}

One of our ambitions is to extrapolate our predictions coming from the complete evolution models not only for the global surface properties but also for the chemical composition. Eqs. (5) and (6) already allow to extrapolate rather accurately the core mass and surface luminosity from our last computed models towards the end of the TP-AGB phase. For the $6 M_{\odot}\left(5 M_{\odot}\right)$ star with $Z=0.02$ (0.005), independent fits of $L$ and $M_{\mathrm{C}}$ as a function of time have been calculated. We also need fits for the surface radius (in order to follow the mass loss rate increase through Eq. (1)) as well as the temperature and density at the base of the convective envelope (to follow the HBB nucleosynthesis). We found, on grounds of the last four 
Table 4. Some features of our TP-AGB models at the end of the complete stellar evolution computations. We indicate the total number $n_{\mathrm{p}}$ of computed thermal pulses. The core mass $\left(M_{\mathrm{C}}\right)-$ luminosity $(L)$ relations $L=\alpha\left(M_{\mathrm{C}}-\beta\right)$ indicated for the $5 M_{\odot}$ $\left(4 M_{\odot}\right)$ star with $Z=0.02(0.005)$ is marginally valid as these stars already experience significant hot-bottom burning. No such a relation is given for the $6 M_{\odot}\left(5 M_{\odot}\right)$ object with $Z=0.02(0.005)$. We refer to the text for the fitting core mass-luminosity relation found for the other stars. $M_{\text {env }}$ is the envelope mass. We also indicate the intensity of the last computed thermal pulse (in term of the maximum luminosity $L_{\mathrm{He}}^{\max }$ produced by the HeBS during this thermal runaway), the duration of the last inter-pulse $\left(\Delta t_{\mathrm{ip}}\right)$ and pulse $\left(\Delta t_{\mathrm{p}}\right)$, the time $\Delta t_{\mathrm{pm}}$ elapsed between the maximum extent of the convective tongue of the last thermal pulse and its disappearance, the maximum mass extension of the convective tongue $\left(\Delta m_{\mathrm{p}}\right)$ and the mass overlap between the last two thermal pulses $\left(\Delta m_{\mathrm{pr}}\right)$. We finally mention the occurrence of the third dredge-up

\begin{tabular}{|c|c|c|c|c|c|c|c|}
\hline & \multicolumn{4}{|c|}{$Z=0.02$} & \multicolumn{3}{|c|}{$Z=0.005$} \\
\hline & $3 M_{\odot}$ & $4 M_{\odot}$ & $5 M_{\odot}$ & $6 M_{\odot}$ & $3 M_{\odot}$ & $4 M_{\odot}$ & $5 M_{\odot}$ \\
\hline$t_{\text {end }}-t_{0}(\mathrm{yr})$ & $9.9410^{5}$ & $1.1310^{5}$ & $4.5610^{4}$ & $2.3210^{4}$ & $4.3910^{5}$ & $5.5310^{4}$ & $2.1310^{4}$ \\
\hline$n_{\mathrm{p}}$ & 19 & 13 & 13 & 14 & 8 & 9 & 9 \\
\hline$L\left(L_{\odot}\right)$ & 9750 & 18200 & 24150 & 33650 & 9250 & 19700 & 27600 \\
\hline$M_{\mathrm{env}}\left(M_{\odot}\right)$ & 1.662 & 2.718 & 3.642 & 4.575 & 1.869 & 2.547 & 3.124 \\
\hline$M_{\mathrm{C}}\left(M_{\odot}\right)$ & 0.6052 & 0.7563 & 0.8290 & 0.9022 & 0.6411 & 0.8277 & 0.9101 \\
\hline$\alpha$ & see rel. & see rel. & 264550 & - & see rel. & 383550 & - \\
\hline$\beta$ & see rel. & see rel. & 0.73729 & - & see rel. & 0.77564 & - \\
\hline$L_{\mathrm{He}}^{\max }\left(L_{\odot}\right)$ & $2.110^{7}$ & $1.210^{7}$ & $5.110^{6}$ & $1.910^{6}$ & $2.410^{7}$ & $6.210^{6}$ & $1.110^{6}$ \\
\hline$\Delta t_{\mathrm{ip}}(\mathrm{yr})$ & 38000 & 9200 & 3700 & 1600 & 45000 & 7500 & 2500 \\
\hline$\Delta t_{\mathrm{p}}(\mathrm{yr})$ & 95 & 24 & 14 & 9 & 104 & 20 & 13 \\
\hline$\Delta t_{\mathrm{pm}}(\mathrm{yr})$ & 19 & 10 & 7 & 6 & 20 & 9 & 8 \\
\hline$\Delta m_{\mathrm{p}}\left(M_{\odot}\right)$ & 0.0115 & 0.0038 & 0.0021 & 0.0011 & 0.0119 & 0.0031 & 0.0015 \\
\hline$\Delta m_{\mathrm{pr}}\left(M_{\odot}\right)$ & 0.0066 & 0.0019 & 0.0010 & 0.0005 & 0.0069 & 0.0017 & 0.0008 \\
\hline 3DUP ? & no ... & no $\ldots$ & no & no & no ... & yes & no \\
\hline
\end{tabular}

Table 5. Comparison between predictions for the rate of HBS advance in mass from Eq. (6) and those coming from our computed models. The mean hydrogen mass fraction $\bar{X}$ and HBS luminosity $L_{\mathrm{H}}$ are taken during the inter-pulse phase of the last computed thermal pulse for each star. $\mathrm{d} M_{\mathrm{C}} / \mathrm{d} t$ is correspondingly calculated over the same inter-pulse phase

\begin{tabular}{|c|c|c|c|c|c|c|c|}
\hline & \multicolumn{4}{|c|}{$Z=0.02$} & \multicolumn{3}{|c|}{$Z=0.005$} \\
\hline & $3 M_{\odot}$ & $4 M_{\odot}$ & $5 M_{\odot}$ & $6 M_{\odot}$ & $3 M_{\odot}$ & $4 M_{\odot}$ & $5 M_{\odot}$ \\
\hline$\overline{\bar{X}}$ & 0.49 & 0.54 & 0.52 & 0.64 & 0.54 & 0.58 & 0.69 \\
\hline$L_{\mathrm{H}}\left(L_{\odot}\right)$ & 3450 & 10690 & 14480 & 17250 & 4600 & 10320 & 12890 \\
\hline Eq. (6) prediction & $6.710^{-8}$ & $1.910^{-7}$ & $2.710^{-7}$ & $2.610^{-7}$ & $8.110^{-8}$ & $1.710^{-7}$ & $1.810^{-7}$ \\
\hline $\mathrm{d} M_{\mathrm{C}} / \mathrm{d} t\left(M_{\odot} \mathrm{yr}^{-1}\right)$ & $6.410^{-8}$ & $1.710^{-7}$ & $2.510^{-7}$ & $3.110^{-7}$ & $7.810^{-8}$ & $1.810^{-7}$ & $2.410^{-7}$ \\
\hline
\end{tabular}

computed thermal pulses for each star, very good fitting relations for these quantities, which are presented in Table 6 . Note that they are linear with time during the last part of the TP-AGB phase, contrarily to $M_{\mathrm{C}}$ and $L$ that increase exponentially during this phase.

The main interest of such an extrapolation procedure is that very detailed stellar evolution computations (including a very complete description of the nucleosynthesis) would take prohibitive computation time. However, we had to make two important approximations (compared to full evolution models) that must be mentioned.
- The successive 3DUP events progressively pollute the convective envelope in ${ }^{4} \mathrm{He},{ }^{12} \mathrm{C}$ and heavier elements to the detriment of ${ }^{1} \mathrm{H}$. This increases the mean molecular weight (or $Z$ ) and the mean opacity in the subphotospheric region, probably leading to an overestimation of the surface luminosity.

- Very few computations are existing for the very end of the TP-AGB phase (Vassiliadis \& Wood 1993; Blöcker 1995), that cannot maintain to give accurate predictions concerning the evolution of the surface isotopic composition. One of their interests is to indicate that 
the last few thermal pulses seem to have different properties than the full amplitude ones (e.g. longer interpulse durations). On the other hand, it has been noted by Boothroyd \& Sackmann (1988a) that the increasing mass loss rate and $Z$ possibly prevent the 3 DUP to occur after these last thermal pulses. As a consequence, assuming the full amplitude regime up to the AGB tip probably makes our following predictions quite indefinite concerning the very end of the TP-AGB phase.

Such extrapolation procedures have already been realized by Groenewegen \& de Jong (1993 and following papers). These synthetic evolution models are very powerful to compare observations with computed models concerning the evolution of the global surface properties of AGB stars. However, they contain shortcomings that do not allow to predict in detail the evolution of the surface isotopic composition. Mainly, (i) only the most abundant elements are followed $\left({ }^{4} \mathrm{He},{ }^{12} \mathrm{C},{ }^{13} \mathrm{C},{ }^{14} \mathrm{~N}\right.$ and $\left.{ }^{16} \mathrm{O}\right)$ and $(i i)$ the $\mathrm{HBB}$ nucleosynthesis is not followed in detail whereas it becomes a very influent process concerning the convective envelope composition towards the TP-AGB tip. Recent progresses have been made by Busso et al. (1995) and Lambert et al. (1995) to follow in detail the nucleosynthesis as late as possible along the AGB phase.

In Sect. 8, we both present the nucleosynthesis encountered in our full evolution models and the extension of the predictions concerning the convective envelope composition towards the end of the AGB phase corresponding to the seven computed stars. For this extrapolation, we proceed as follows.

1. From the last computed inter-pulse phase for each star, we integrate, during the successive inter-pulse phases, the evolution of the nucleosynthesis occuring inside the convective envelope by solving Eqs. (4), suited for the $\mathrm{HBB}$, for each nuclide while imposing a temperature and density increases at the bottom of the convective envelope given by the fits presented in Table 6 . We took the inter-pulse durations of Table 4; indeed, they are only very slightly decreasing with the core mass increase along the asymptotic TP-AGB phase.

2. Since the $3 \mathrm{DUP}$ is almost ready to occur in our models (see Sect. 6.2), we mix the envelope with the HBS and inter-shell regions recurrently, every $\Delta t_{\text {ip }}$. To quantify the adopted depth of each $3 \mathrm{DUP}$, let us remember the definition of the usual parameter

$\lambda=\frac{\Delta M_{\mathrm{DUP}}}{\Delta M_{\mathrm{C}}}$,

where $\Delta M_{\text {DUP }}$ represents the amount of mass that is dredged-up (difference between the normal depth of the convective envelope and its maximum downwards penetration during a dredge-up) and $\Delta M_{\mathrm{C}}$ the increase in mass of the HBS between two successive thermal pulses. We adopted a 3DUP depth corresponding to $\lambda=0.6$ for all the stars. This value has been suggested by Groenewegen \& de Jong (1993) because it repro- duces rather well the carbon star luminosity function of the LMC (that cannot be fitted on grounds of selfconsistent evolution models only; see Sect. 6.2).

During such a dredge-up, we assumed that the material coming from the convective tongue of a thermal pulse has abundance profiles corresponding to end of the last computed thermal pulse. Of course, we cannot do better without further computing thermal pulses with detailed nucleosynthesis up to the end of the TPAGB phase that is, again, not yet realistic. However, this appears to be rather justified for many important chemical elements as they reach almost constant abundances after the few last computed thermal pulses (see discussion in Sect. 7).

Taking a constant value for $\lambda$ is probably not strictly right. As far as the surface composition is concerned, increasing $\lambda$ more efficiently pollutes the convective envelope from pulse to pulse.

There is another quantity that greatly influences the evolution of the envelope composition: the mass loss rate. Indeed, decreasing the mass loss rate allows to increase the total number of thermal pulses (and consequently the total number of 3DUP episodes) before the end of the TPAGB phase. We had to increase our $\eta$ values in Eq. (1) for each star, in order to reach the highest mass loss rates (called super-winds) observed among very evolved AGB stars (see the discussion in Sect. 2.1.5; see also Zuckerman et al. 1986; Wannier \& Sahai 1986; Knapp et al. 1989): we adopted $\eta=5,10,15$ and 20 for the $3,4,5$ and $6 M_{\odot}$ AGB models, respectively, whatever $Z$. As the exact way following which the mass loss rate increases with time along the asymptotic TP-AGB phase is still questionable by more than a factor of two (see Sect. 2.1.5), the resulting uncertainty on the evolution of the surface composition up to the AGB tip dominates the effect of $\lambda$ (see our discussion in Sect. 8.1, where our mass loss rates are varied by a factor of four).

In Table 7, we present the global mass loss increases we obtained, for the seven TP -AGB stars, from the beginning of the TP-AGB phase up to its end (i.e. including our extrapolation computations).

\subsubsection{Initial mass-final mass relation}

One of the most important constraint for evolutionary models towards the AGB tip is the reproduction of the observed correlation between the initial mass of stars $\left(M_{\mathrm{i}}\right)$ and their final one $\left(M_{\mathrm{f}}\right)$, i.e. their core mass (become a hot white dwarf) when the convective envelope has been completely removed after the planetary nebula (PN) ejection. We present in Fig. 2 the comparison between observations analyzed by Weidemann \& Koester (1983) and Weidemann (1987) and the final core masses we obtained after our evolutionary plus extrapolations computations. We note that agreement with observations is slightly better than for Vassiliadis \& Wood (1993), especially around 
Table 6. Fitting parameters from the last four computed asymptotic thermal pulses towards the end of the TP-AGB phase, for the mean total radius and bottom temperature and density of the convective envelope. For each quantity $A$, the relation reads $A(t)=\alpha\left(t-t_{0}\right)+\beta$, with time in yr

\begin{tabular}{|c|c|c|c|c|c|c|c|c|}
\hline & & \multicolumn{4}{|c|}{$Z=0.02$} & \multicolumn{3}{|c|}{$Z=0.005$} \\
\hline & & $3 M_{\odot}$ & $4 M_{\odot}$ & $5 M_{\odot}$ & $6 M_{\odot}$ & $3 M_{\odot}$ & $4 M_{\odot}$ & $5 M_{\odot}$ \\
\hline$\overline{\bar{R}\left(t-t_{0}\right)\left(R_{\odot}\right)}$ & $\begin{array}{l}\alpha \\
\beta\end{array}$ & $\begin{array}{l}3.1710^{-4} \\
8.5310^{1}\end{array}$ & $\begin{array}{l}1.1610^{-3} \\
4.1110^{2}\end{array}$ & $\begin{array}{l}1.7610^{-3} \\
5.1110^{2}\end{array}$ & $\begin{array}{l}1.9510^{-3} \\
6.3510^{2}\end{array}$ & $\begin{array}{l}9.0310^{-5} \\
2.3710^{2}\end{array}$ & $\begin{array}{l}1.3310^{-3} \\
3.5710^{2}\end{array}$ & $\begin{array}{l}3.4810^{-3} \\
4.0610^{2}\end{array}$ \\
\hline$\overline{T_{\mathrm{cb}}}\left(t-t_{0}\right)(\mathrm{K})$ & $\begin{array}{l}\alpha \\
\beta\end{array}$ & $\begin{array}{l}1.8110^{-1} \\
4.0510^{6}\end{array}$ & $\begin{array}{l}2.8810^{1} \\
1.1410^{7}\end{array}$ & $\begin{array}{l}5.1310^{2} \\
2.0510^{7}\end{array}$ & $\begin{array}{l}4.2110^{2} \\
6.3410^{7}\end{array}$ & $\begin{array}{l}3.53 \\
3.1610^{6}\end{array}$ & $\begin{array}{l}2.8110^{2} \\
8.8210^{6}\end{array}$ & $\begin{array}{l}1.0710^{3} \\
4.5010^{7}\end{array}$ \\
\hline$\overline{\rho_{\mathrm{cb}}}\left(t-t_{0}\right)(\mathrm{cgs})$ & $\begin{array}{l}\alpha \\
\beta\end{array}$ & $\begin{array}{l}1.1610^{-10} \\
1.7210^{-3}\end{array}$ & $\begin{array}{l}2.5110^{-7} \\
2.2810^{-2}\end{array}$ & $\begin{array}{l}1.5610^{-5} \\
5.4010^{-2}\end{array}$ & $\begin{array}{l}3.3210^{-5} \\
2.47\end{array}$ & $\begin{array}{l}4.7110^{-9} \\
9.8210^{-4}\end{array}$ & $\begin{array}{l}3.8610^{-6} \\
1.0410^{-3}\end{array}$ & $\begin{array}{l}1.2410^{-4} \\
3.5310^{-1}\end{array}$ \\
\hline
\end{tabular}

Table 7. Increase of the mass loss rates during the whole TP-AGB phase

\begin{tabular}{|c|c|c|c|c|c|c|c|c|}
\hline & & \multicolumn{4}{|c|}{$Z=0.02$} & \multicolumn{3}{|c|}{$Z=0.005$} \\
\hline & & $3 M_{\odot}$ & $4 M_{\odot}$ & $5 M_{\odot}$ & $6 M_{\odot}$ & $3 M_{\odot}$ & $4 M_{\odot}$ & $5 M_{\odot}$ \\
\hline \multirow[t]{2}{*}{$\bar{M}\left(M_{\odot} \mathrm{yr}^{-1}\right)$} & $a t t_{0}$ & $2.010^{-7}$ & $1.510^{-6}$ & $2.510^{-6}$ & $3.610^{-6}$ & $3.610^{-7}$ & $1.810^{-6}$ & $2.810^{-6}$ \\
\hline & $A G B$ tip & $7.610^{-6}$ & $4.310^{-5}$ & $9.410^{-5}$ & $1.510^{-4}$ & $5.610^{-6}$ & $4.010^{-5}$ & $1.010^{-4}$ \\
\hline
\end{tabular}

$M_{\mathrm{i}}=3 M_{\odot}$ where they only reproduce the upper limits. On the contrary, the final core mass obtained by Boothroyd \& Sackmann (1988b) is rather low compared to observations. Note we have not taken into account of the core mass reduction following each 3DUP event. However, as explained below, such a reduction, even if it increases the agreement with the observed relation, is rather negligible compared to other uncertainties (core mass at the first thermal pulse, thermal pulse number depending on the adopted mass loss rate, ...).

We observe that changing the mass loss rate by a factor of four during the asymptotic TP-AGB phase (see also Sect. 8.1 concerning surface abundance predictions) does not significantly change the agreement with observations. We claim that this is due to the fact that while almost $50 \%$ of the $\mathrm{C}-\mathrm{O}$ core mass is built during the central Heburning phase, roughly 35 to $45 \%$ (in mass) are added during the E-AGB phase. More precisely, during the whole TP-AGB phase, we found that our core masses increased by $14(12), 7(4), 5(3)$ and $4 \%$ for the $3,4,5$, and $6 M_{\odot}$ stars with $Z=0.02(0.005)$, respectively. The final core mass is consequently almost decided before the super-wind occurs and this observed relation is not very compulsive for the empirical mass loss rate to adopt during the TPAGB phase.

\section{Nucleosynthesis during TP-AGB phase}

From the pioneer work of Iben (1975), one realized that inside TP-AGB stars, a very rich nucleosynthesis occurs. During the recurrent third dredge-up events, the synthesized nuclides are mixed inside the convective envelope and then ejected into the interstellar medium (ISM) through the strong mass loss. In conclusion, TP-AGB stars play a crucial role for the chemical evolution of galaxies, especially for some elements like ${ }^{3} \mathrm{He},{ }^{7} \mathrm{Li},{ }^{19} \mathrm{~F}$, ${ }^{26} \mathrm{Al}$, nuclides heavier than ${ }^{56} \mathrm{Fe}, \ldots$ It is the reason why we will give, in Sect. 8.3, the yields resulting from our intermediate-mass star computations.

\subsection{Overview of the various nucleosynthesis sites}

Let us analyze the various nucleosynthesis processes occurring inside intermediate-mass TP-AGB stars. In fact, three very different sites have to be distinguished.

\subsubsection{Burning shells}

As indicated in Table 2, most of the nuclear energy release on the AGB is due to the HBS, mainly through the CNO bi-cycle. More precisely, the six most important nuclear reactions that energetically supply the star are ${ }^{13} \mathrm{C}(\mathrm{p}, \gamma)$ $(\sim 26 \%), \quad{ }^{14} \mathrm{~N}(\mathrm{p}, \gamma) \quad(\sim 24 \%),{ }^{15} \mathrm{~N}(\mathrm{p}, \alpha) \quad(\sim 17 \%),{ }^{15} \mathrm{O}$ $\left(\beta^{+}\right)(\sim 9 \%),{ }^{13} \mathrm{~N}\left(\beta^{+}\right)(\sim 7 \%)$ and ${ }^{12} \mathrm{C}(\mathrm{p}, \gamma)(\sim 6 \%)$, all occurring inside the HBS. These numbers are quite similar (within a few \%) for all our modeled stars. Whatever 


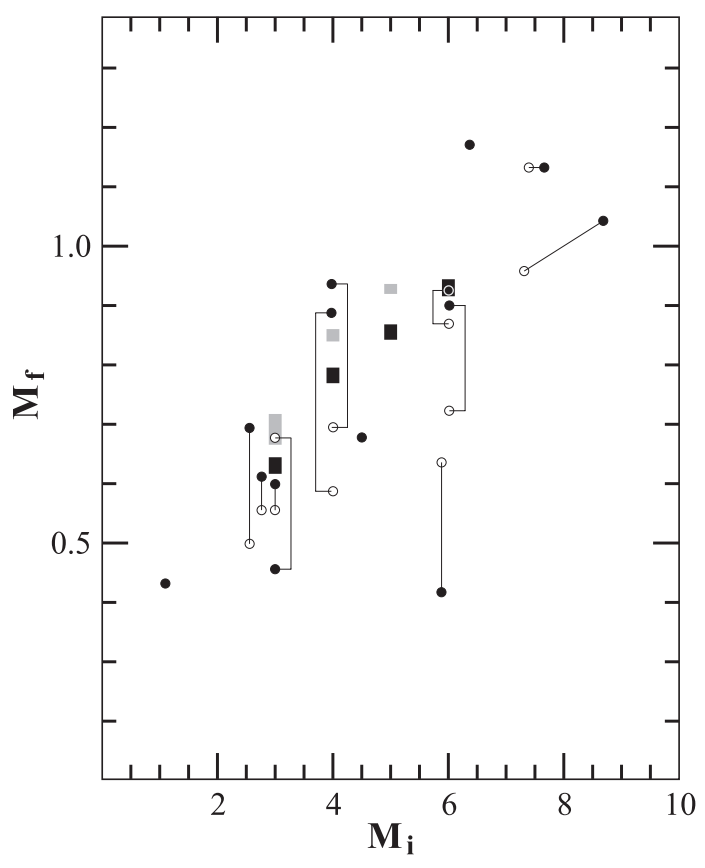

Fig. 2. Relation between the initial mass of stars and the final white dwarf (WD) mass at the end of the AGB phase. Observational final masses are derived either from the surface gravity of the WD (filled circle) or from their radius (open circles); lines are connecting both determinations for identical objects. Our predictions are the black and gray rectangles, for the $Z=0.02$ and $Z=0.005$ modeled stars, respectively. Their extension is the result of varying the mass loss rate by a factor of four during the asymptotic TP-AGB phase

the AGB star total mass, the HBS slightly depletes ${ }^{12} \mathrm{C}$ and ${ }^{15} \mathrm{~N}$ and significantly depletes ${ }^{17} \mathrm{O},{ }^{18} \mathrm{O}$ and ${ }^{19} \mathrm{~F}$, while ${ }^{14} \mathrm{~N}$ is produced. The case of ${ }^{13} \mathrm{C}$ is more difficult: while its abundance is very slightly increased in the HBS of $3 M_{\odot}$ stars (whatever $Z$ ), it is somewhat decreased in the HBS of $6 M_{\odot}$ stars.

The rather high temperature inside the HBS also allows the activation of the $\mathrm{NeNa}$ and $\mathrm{MgAl}$ chains, even if they are much less influent for the global energetics. The most interesting consequences of the NeNa chain are the production of ${ }^{22} \mathrm{Ne}$ and ${ }^{23} \mathrm{Na}$ in $\leq 5 M_{\odot}$ stars and their slight destruction in more massive AGB stars. The $\mathrm{MgAl}$ chain is very sensitive to the AGB total mass. In $<5 M_{\odot}$ objects, ${ }^{25} \mathrm{Mg}$ is destroyed to the benefit of ${ }^{26} \mathrm{Al}$ (roughly half in ${ }^{26} \mathrm{Al}^{\mathrm{g}}$ and half in ${ }^{26} \mathrm{Al}^{\mathrm{m}}$ that quite instantaneously decays in ${ }^{26} \mathrm{Mg}$ ); a small quantity of ${ }^{26} \mathrm{Mg}$ is also destroyed in ${ }^{27} \mathrm{Al}$. In more massive AGB stars, the HBS temperature is high enough to substantially produce ${ }^{25} \mathrm{Mg}$ through the ${ }^{24} \mathrm{Mg}(\mathrm{p}, \gamma)$ reaction. This in turn leads to a much important ${ }^{26} \mathrm{Al}^{\mathrm{g}}$ production. However, as the HBS mean temperature always increases with time, ${ }^{26} \mathrm{Al}^{\mathrm{g}}$ begins to be partially destroyed by $(\mathrm{p}, \gamma)$ reactions late along the TP-AGB. We refer to Forestini et al. (1991) for the first detailed study of ${ }^{26} \mathrm{Al}^{\mathrm{g}}$ production inside AGB stars, by radiative proton burning (i.e. in the HBS). In Guelin et al. (1995), more details can be found, including the possibility to synthesize ${ }^{26} \mathrm{Al}^{\mathrm{g}}$ by proton burning at the bottom of the convective envelope (i.e. by HBB). It was however a parametric study. First predictions concerning the enrichment of the AGB star surfaces, on grounds of full evolutionary models are presented in Sects. 7.6 and 8.1.

The comparatively small amount of nuclear energy supplied by the HeBS comes from the $3 \alpha(\sim 70 \%)$ and ${ }^{12} \mathrm{C}(\alpha, \gamma) \quad(\sim 29 \%)$ reactions. Consequently, the HeBS mainly produces ${ }^{12} \mathrm{C}$ and, to a lower extent, ${ }^{16} \mathrm{O}$, while during the previous central He-burning stage, the ${ }^{16} \mathrm{O}$ production was preponderant. At the beginning of a thermal pulse, it is the $3 \alpha$ reaction that becomes the dominant energy source (see also Sect. 7.1.3 for the thermal pulse energetics).

During the long inter-pulse phase that follows a 3DUP episode, the ${ }^{13} \mathrm{C}$ coming out of the HBS can be burnt in the inter-shell region, close to the HeBS top, mainly through the ${ }^{13} \mathrm{C}(\alpha, \mathrm{n}){ }^{16} \mathrm{O}$ reaction. This reaction is highly energetic. The first published work reporting the existence of this inter-shell ${ }^{13} \mathrm{C}$ burning is Straniero et al. (1995). The produced neutrons are then captured by light species up to ${ }^{56} \mathrm{Fe}$ mainly, but part on them are also used to synthesize $s$ elements. By decreasing order of amplitude, the nuclear energy liberated in the inter-shell region during the inter-pulse phase comes from these $(\mathrm{n}, \gamma)$ reactions up to ${ }^{56} \mathrm{Fe}(\sim 29 \%),{ }^{13} \mathrm{C}(\alpha, \mathrm{n}) \quad(\sim 21 \%)$, the so-called sprocess reactions $(\sim 12 \%),{ }^{26} \mathrm{Al}^{\mathrm{g}}(\mathrm{n}, \mathrm{p}){ }^{26} \mathrm{Mg}(\sim 7 \%)$ and ${ }^{14} \mathrm{~N}(\mathrm{n}, \mathrm{p}){ }^{14} \mathrm{C}(\sim 2.5 \%)$. Note the important nuclear energy released by the neutron captures. This energy production creates a third burning shell between the HeBS and HBS, even if its nuclear activity is considerably lower than that of both the HeBS and HBS. It continues to exist until ${ }^{13} \mathrm{C}$ has been destroyed there. This happens, in general, almost when the following thermal pulse begins. As a consequence, when the convective tongue of a thermal pulse engulfs the inter-shell matter, the amount of ${ }^{13} \mathrm{C}$ that is mixed is reduced compared to what was previously thought, i.e. $\Delta m[\mathrm{H}-\mathrm{He}] X\left({ }^{13} \mathrm{C}\right)[\mathrm{H}-\mathrm{He}]$, where $[\mathrm{H}-\mathrm{He}]$ designates, as before, the inter-shell region. We show, in Fig. 3 , the nuclear energy production rate $\left(\varepsilon_{\text {nuc }}\right)$ profile in the case of our $3 M_{\odot}$ AGB star. This clearly demonstrates the three-burning shell structure. Such a structure is also found in the more massive AGB stars. Let us emphasize that this third burning shell could at least partially explain the production of the $s$ nuclides (see Sect. 7.5) in a radiative zone. This has been suggested by Straniero et al. (1995), even if their amount of ${ }^{13} \mathrm{C}$ in the inter-shell was artificially increased in order to study the s-process nucleosynthesis. 


\section{$\mathrm{M}_{\text {ini }}=3 \mathrm{M}_{\odot} ; \mathrm{Z}=\mathbf{0 . 0 2}$ INTERPULSE 18-19}

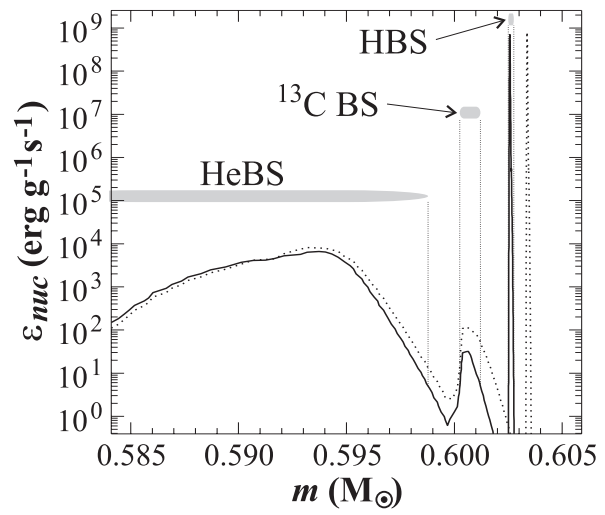

Fig. 3. Profile of the nuclear energy production rate per unit mass, $\varepsilon_{\text {nuc }}$, in the region of the burning shells, in the case of the $3 M_{\odot}(Z=0.02)$ star, during the inter-pulse phase between the 18th and the 19th thermal runaways. The dotted curve corresponds to a time just before the 19th thermal pulse, while the solid one is plotted roughly at the middle of the inter-pulse phase (see the text for a discussion)

\subsubsection{Hot-bottom burning}

If the temperature $T_{\mathrm{cb}}$ at the base of the deep convective envelope is high enough (i.e. $>2010^{6} \mathrm{~K}$ ), i.e. if the base of the convective envelope reaches the HBS, proton burning occurs inside it. This HBB occurs in models with initial masses $\geq 5 M_{\odot}$ for $Z=0.02$ and $\geq 4 M_{\odot}$ for $Z=0.005$. These lower mass limits and this $Z$ dependence are common features of all the AGB models (see e.g. Boothroyd et al. 1993). As mixing up to the cool surface is very efficient (the mean turn-over time $\tau_{\mathrm{CONV}}$ of the convective envelope is roughly $0.5 \mathrm{yr}$ ), the effective nuclear reaction rates are lower than in radiative $\mathrm{H}$-Burning, leading to specific nucleosynthesis signatures.

For $T_{\mathrm{cb}}>4010^{6} \mathrm{~K}$ already, ${ }^{7} \mathrm{Li}$ can be produced from ${ }^{3} \mathrm{He}$ following a scenario that cannot operate in radiative H-burning (see Sect. 7.2 for more details). This Li synthesis has been qualitatively suggested by Cameron \& Fowler (1971) and quantitatively first demonstrated on grounds of evolutionary models by Sackmann \& Boothroyd (1992).

For $T_{\mathrm{cb}}>6010^{6} \mathrm{~K}$, the $\mathrm{CN}$ cycle begins to operate partially, i.e. ${ }^{12} \mathrm{C}$ is converted to ${ }^{13} \mathrm{C}$ that accumulates. As first investigated by Boothroyd et al. (1993), this conversion can efficiently reduce the ${ }^{12} \mathrm{C} /{ }^{16} \mathrm{O}$ ratio and eventually, if HBB is strong enough, this can prevent the formation of C stars (see also Sects. 8.1 and 8.2).

If $T_{\mathrm{cb}}>8010^{6} \mathrm{~K}$ typically, the $\mathrm{CN}$ cycle almost operates at equilibrium through the whole envelope, leading to $\mathrm{a}^{12} \mathrm{C} /{ }^{13} \mathrm{C}$ isotopic ratio lower than 10 (depending on $T_{\mathrm{cb}}$ ). In our models, this happens for the $6 M_{\odot}\left(5 M_{\odot}\right) \mathrm{AGB}$ star with $Z=0.02(0.005)$ and also, later along the TPAGB, for our $5 M_{\odot}$ star with $Z=0.02$ (see Sect. 8.1). At very high $T_{\mathrm{cb}}$, other reactions begin to occur; in particular, in extreme cases (i.e. the end of the TP-AGB phase of our $6 M_{\odot}$ models with $\left.Z=0.02\right),{ }^{26} \mathrm{Al}^{\mathrm{g}}$ can eventually be directly produced inside the convective envelope through the ${ }^{25} \mathrm{Mg}(\mathrm{p}, \gamma)$ reaction on ${ }^{25} \mathrm{Mg}$ supplied by ${ }^{24} \mathrm{Mg}(\mathrm{p}, \gamma)$ (see Sect. 8.1).

\subsubsection{Convective tongue of thermal pulses}

The thermal runaway, inside the HeBS, leading to form a convective tongue is principally due to the $3 \alpha$ reaction excitement. When this convective tongue finally penetrates the inter-shell region, the HBS ashes are ingested, i.e. not only ${ }^{4} \mathrm{He}$ but also ${ }^{13} \mathrm{C},{ }^{14} \mathrm{~N}$ and ${ }^{26} \mathrm{Al}{ }^{\mathrm{g}}$ mainly. As these elements are partially burnt in the bottom of the inter-shell region during the inter-pulse phase (globally, we find that half of the ${ }^{13} \mathrm{C}$ is so destroyed), they mainly survive in the upper part of the inter-shell. They are consequently engulfed by the convective tongue when it reaches its maximum extension. At that time, the $3 \alpha$ reaction is already dominant for the energetics. We consequently find that the energetics associated with the ${ }^{13} \mathrm{C}$ burning through the ${ }^{13} \mathrm{C}(\alpha, \mathrm{n})$ reaction as well as the neutron captures that follow is rather negligible (less than 1\%). This is also found by Straniero et al. (1995). This conclusion could however be modified if more ${ }^{13} \mathrm{C}$ was synthesized in the inter-shell region, as required for the s-process (see Sect. 7.5). The important energetic effects reported by Bazan \& Lattanzio (1993) concerning the ${ }^{13} \mathrm{C}$ burning inside thermal pulses was probably due to (i) the fact they did not give account of partial inter-shell ${ }^{13} \mathrm{C}$ burning and (ii) the very high ${ }^{13} \mathrm{C}$ amount artificially ingested into their thermal pulses in order to study the consequent s-process.

Part of produced neutrons participate to (n, p) reactions, mainly on ${ }^{26} \mathrm{Al}^{\mathrm{g}}$ and, to a lower extent, on ${ }^{14} \mathrm{~N}$. The resulting protons, at these high temperatures characterizing He-burning during a thermal pulse (>200106 $\mathrm{K}$ at the base of the convective tongue), are rapidly captured by various elements (see Sects. 7.3 to 7.5 for more details). Let us stress that the simultaneous presence, inside a mixed He-burning region (the convective tongue), of neutrons and protons leads to a very specific (and unique) nucleosynthesis.

We will analyze in more details the nucleosynthesis processes associated with the thermal pulses in Sects. 7.3 to 7.5 .

\subsection{Lithium production}

Sackmann \& Boothroyd (1992) were the first ones to quantitatively demonstrate how the Cameron \& Fowler (1971) scenario leads to ${ }^{7} \mathrm{Li}$ synthesis in intermediate-mass AGB stars. This major result was obtained by using a time-dependent convective diffusion algorithm suited for hot-bottom convective envelopes. Indeed, at the base of such envelopes, some nuclear reactions involved in ${ }^{7} \mathrm{Li}$ 
production occur faster than mean turn-over time associated with the convective motions (see Fig. 4).

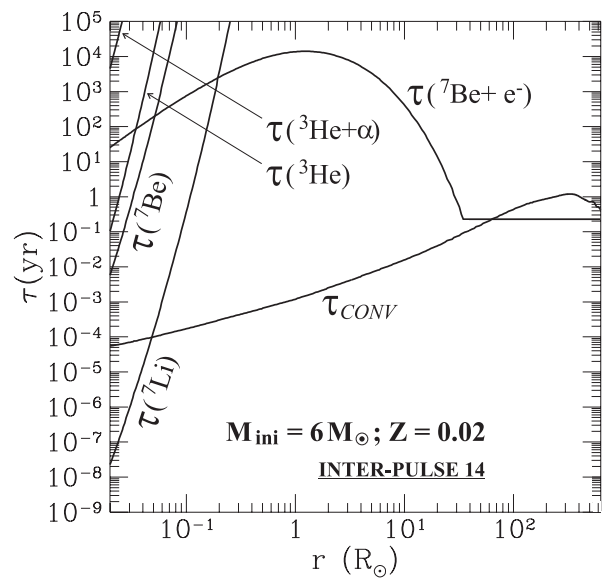

Fig. 4. Nuclear reaction time scales for the reactions involved in the Cameron-Fowler scenario, inside the convective envelope of the $6 M_{\odot}(Z=0.02)$ star during the 14 th inter-pulse. At that time, its base temperature is $\simeq 7510^{6} \mathrm{~K} . \tau\left({ }^{7} \mathrm{Be}\right)$ and $\tau\left({ }^{7} \mathrm{Li}\right)$ correspond to the proton capture on ${ }^{7} \mathrm{Be}$ and ${ }^{7} \mathrm{Li}$, respectively. $\tau\left({ }^{3} \mathrm{He}\right)$ and $\tau\left({ }^{3} \mathrm{He}+\alpha\right)$ relate to the ${ }^{3} \mathrm{He}$ destruction by ${ }^{3} \mathrm{He}$ and ${ }^{4} \mathrm{He}$ capture, respectively. Finally, $\tau\left({ }^{7} \mathrm{Be}+\mathrm{e}^{-}\right)$is the electron capture rate on ${ }^{7} \mathrm{Be}$. The mean turn-over time of the convective motions is also plotted $\left(\tau_{\mathrm{CONV}}\right)$

We also use a time-dependent treatment to study the ${ }^{7} \mathrm{Li}$ synthesis (see Eqs. (4) in Sect. 2.2.3. We show in Fig. 5 the typical resulting ${ }^{7} \mathrm{Be}$ and ${ }^{7} \mathrm{Li}$ profiles inside the convective envelope of our $6 M_{\odot}$ AGB models with $Z=0.02$. When HBB occurs (see Sect. 7.1.2), ${ }^{3} \mathrm{He}$ first begins to burn through the ${ }^{3} \mathrm{He}\left({ }^{3} \mathrm{He}, 2 \mathrm{p}\right)$ and ${ }^{3} \mathrm{He}(\alpha, \gamma){ }^{7} \mathrm{Be}$ reactions, the second one being significantly slower than the first one. As in deep stellar interiors, the ${ }^{7} \mathrm{Be}$ destruction by electron capture and proton capture are much longer than the time scale associated with the convective mixing, the produced ${ }^{7} \mathrm{Be}$ is efficiently up-heaved towards cooler regions where it progressively decays in ${ }^{7} \mathrm{Li}$. Let us emphasize that without fast convective mixing, ${ }^{7} \mathrm{Li}$ could not accumulate at the surface. Indeed, at the base of the convective envelope, (i) ${ }^{7} \mathrm{Be}$ would be partially destroyed by electron and proton captures and (ii) the ${ }^{7} \mathrm{Li}$ resulting from ${ }^{7} \mathrm{Be}$ decay would be quite instantaneously destroyed by proton capture too.

Let us now compare our predictions with Sackmann \& Boothroyd (1992) results and with observations. A direct comparison with their Figs. 2a and $2 \mathrm{~b}$ reveals nearly identical features. In Fig. 6, we present the evolution of the surface ${ }^{7} \mathrm{Li}$ abundance during the TP-AGB phase of all our modeled stars. In our $3 M_{\odot}$ models, the temperature at the base of the convective envelope is never high enough for ${ }^{7} \mathrm{Be}$ to be produced efficiently, and the surface ${ }^{7} \mathrm{Li}$ abundance remains almost constant at a very low

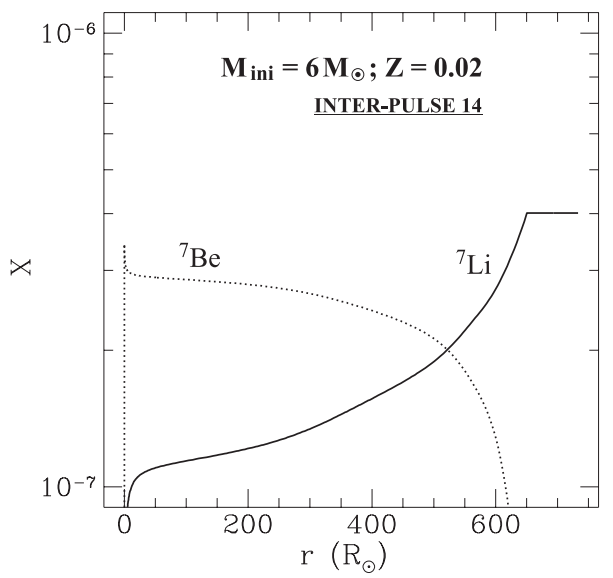

Fig. 5. ${ }^{7} \mathrm{Be}$ and ${ }^{7} \mathrm{Li}$ profiles inside the same convective envelope as in Fig. 4

value resulting from the first dredge-up. We also see that our $4 M_{\odot}$ star with $Z=0.005$ begins to produce some ${ }^{7} \mathrm{Li}$ when it enters the asymptotic regime, but the surface abundance of this light element always stays at least one order of magnitude below the interstellar medium (or cosmic) value $\varepsilon\left({ }^{7} \mathrm{Li}\right)=\log \frac{\mathrm{Li}}{\mathrm{H}}+12 \simeq 3.3$. Note the ${ }^{7} \mathrm{Li}$ destruction inside the $4 M_{\odot}$ star with $Z=0.02$ attesting that its convective bottom temperature is high enough to burn ${ }^{7} \mathrm{Li}$ through ${ }^{7} \mathrm{Li}(\mathrm{p}, \alpha)$ but not enough to produce ${ }^{7} \mathrm{Be}$ by ${ }^{3} \mathrm{He}$ burning. Finally, our 5 and $6 M_{\odot}$ models present real high ${ }^{7} \mathrm{Li}$ surface enhancements, compared to the cosmic value. The maximum abundances we obtain range between $\varepsilon\left({ }^{7} \mathrm{Li}\right) \simeq 4.2$ to 5.1 , depending on the stellar mass and metallicity. Our super-lithium-rich stars are predicted to appear in a luminosity range between $2.610^{4}$ and $3.610^{4} L_{\odot}$, i.e. for $M_{\text {bol }}$ between -6.25 and -6.65 . These values are in good agreement with those found by Sackmann \& Boothroyd (1992).

As the evolution proceeds, the surface ${ }^{7} \mathrm{Li}$ abundances finally decrease again very rapidly. This indicates that ${ }^{3} \mathrm{He}$ has been almost completely burned in the envelope (remember that the ${ }^{3} \mathrm{He}\left({ }^{3} \mathrm{He}, 2 \mathrm{p}\right)$ is dominant), so that the ${ }^{7} \mathrm{Li}$ production can no more be supported and it is then destroyed by proton capture. This fact clearly puts an upper limit in luminosity to observe super-lithium-rich AGB stars. This last feature is also present in Sackmann \& Boothroyd (1992), even if it appears somewhat later during the TP-AGB phase of their most massive objects. This could be due to different mass loss rate prescriptions.

How do these predictions compare with observational data (see Fig. 6)? In the Magellanic Clouds, the large majority of lithium-rich giants are observed within a narrow luminosity range, $M_{\text {bol }} \simeq-6$ to $\simeq-6.9$ (Smith \& Lambert 1989, 1990b; Plez et al. 1993; Smith et al. 1995), in very good agreement with the predictions. The observed abundance range is also well reproduced, even if no star has been observed with $\varepsilon\left({ }^{7} \mathrm{Li}\right)$ higher than 4.5 in 
the Clouds. Moreover, the mass estimate from pulsation theory for some lithium-rich giants of both Clouds is consistent with the predicted mass range for surface ${ }^{7} \mathrm{Li}$ enhancement (Smith et al. 1995). Comparisons with ${ }^{7} \mathrm{Li}$ in galactic giants are more delicate because of the larger observational uncertainties on luminosity and mass. Galactic super-lithium-rich AGB stars can exhibit $\varepsilon\left({ }^{7} \mathrm{Li}\right)$ values as high as 5.4 (Abia et al. 1991), but these carbon stars have $M_{\mathrm{bol}}$ laying between -5 and -6.2 , i.e. rather lower than the predicted luminosities. This could be due to a bad luminosity determination, as it seems difficult to synthesize ${ }^{7} \mathrm{Li}$ inside the convective envelope of AGB stars with such relatively low luminosities. However, most of the galactic super-lithium-rich AGB stars have lower masses than the ones we model here, and they will be addressed in a forthcoming paper.

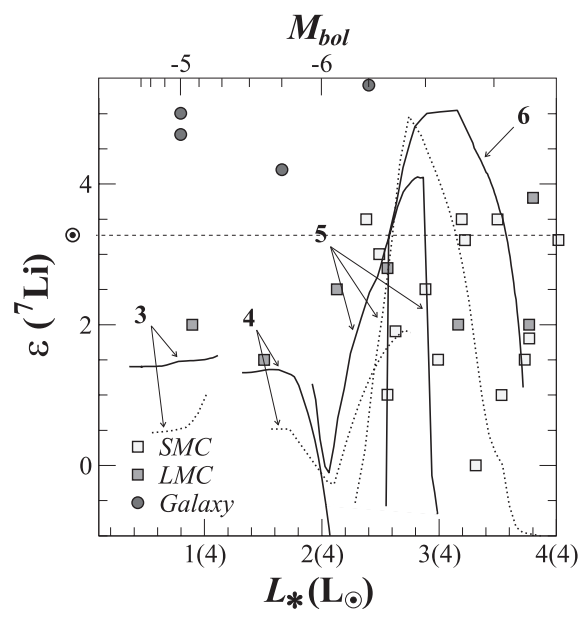

Fig. 6. Resulting surface ${ }^{7} \mathrm{Li}$ abundances, in term of $\varepsilon\left({ }^{7} \mathrm{Li}\right)$, for the seven computed TP-AGB phases, as a function of the stellar surface luminosity. The solid (dotted) lines refer to the $Z=0.02(0.005)$ models. Observations are shown for bright AGB stars of the SMC and LMC (Smith et al. 1995) and of the Galaxy (Abia et al. 1991)

\subsection{CNO nucleosynthesis and Fluorine production}

We now review all the species (from $\mathrm{C}$ ) by indicating in which nuclear reactions they are implicated inside the convective tongue of the successive thermal pulses, as a function of the initial mass $M$ and metallicity $Z$ of the computed TP-AGB models. For the discussion, we refer to Figs. 7 to 9 of the electronic version of the paper. The mean mass fractions $\overline{X_{\mathrm{a}}}$ are calculated by taking the average of the mass fraction profile of each nuclide $a$ over the maximum region covered by the convective tongue of each thermal pulse, just after its disappearance, i.e.

$\overline{X_{\mathrm{a}}}=\frac{1}{\Delta M_{\text {conv. }} \text { tongue }} \underset{\substack{\text { conv. } \\ \text { tongue }}}{ } X_{\mathrm{a}} \mathrm{d} m$.
We also refer to Fig. 7 that presents the mean mass fractions of key nuclides (calculated as for the convective tongue) in each burning region (i.e. the HBS and intershell regions) compared to their convective envelope content, for the $3 M_{\odot}$ star with $Z=0.02$. Similar figures for the other six AGB stars are only available in the electronic version. Similarly, Fig. 8 presents the abundance variations of the same nuclides inside typical convective thermal pulses. Again, similar figures for the other six AGB stars can solely be found in the electronic form. All these figures are supporting the following nucleosynthesis descriptions.

During the growth of the convective tongue, the mean ${ }^{12} \mathrm{C}$ mass fraction first decreases due to the ${ }^{4} \mathrm{He}$ mixing from the inter-shell region. Then, at the thermal pulse maximum, ${ }^{12} \mathrm{C}$ is produced by the $3 \alpha$ reaction and also enhanced due to the downwards penetration of the convective tongue into the He-burnt shell. Although this penetration is commonly found, its amplitude seems somewhat more important in our case, bringing more ${ }^{12} \mathrm{C}$ into the thermal pulses. Note this increased amount of ${ }^{12} \mathrm{C}$ makes the 3DUP episodes more efficient to convert an AGB star into a $\mathrm{C}$ star. During the first thermal pulses, the amount of ${ }^{12} \mathrm{C}$ that is produced increases due to the higher temperatures reached at the base of the convective tongue. This bottom temperature increase from pulse to pulse is a common feature of all the stars and it traduces that the thermal pulse intensity increases. The last computed thermal pulses of the $3 M_{\odot}$ object with $Z=0.02$ are even hot enough to partially destroy ${ }^{12} \mathrm{C}$ by the ${ }^{12} \mathrm{C}(\alpha, \gamma)$ reaction.

Most of the engulfed ${ }^{13} \mathrm{C}$ is destroyed very efficiently through ${ }^{13} \mathrm{C}(\alpha, \mathrm{n})$ that produces neutrons. The trend of the remaining mean ${ }^{13} \mathrm{C}$ mass fraction to increase from pulse to pulse is due to the fact that stronger thermal pulses develop convective tongues reaching regions always closer to the HBS, i.e. engulfing more material from the inter-shell zone. As mentioned in Sect. 6.1, the bottom of the HBS cannot be reached however.

${ }^{14} \mathrm{C}$ is only produced by the capture of part of the neutrons on the engulfed ${ }^{14} \mathrm{~N}$, through ${ }^{14} \mathrm{~N}(\mathrm{n}, \mathrm{p})$. Note that this reaction contributes to the production of protons. For initial masses above $4 M_{\odot}\left(3 M_{\odot}\right)$ with $Z=0.02(0.005)$ however, the convective tongue finally becomes hot enough (base temperature $>26010^{6} \mathrm{~K}$ ) to significantly destroy the produced ${ }^{14} \mathrm{C}$ by the ${ }^{14} \mathrm{C}(\alpha, \gamma)$ reaction. The relatively low ${ }^{14} \mathrm{C}$ production inside the $3 M_{\odot}$ with $Z=0.02$ object is due to the relatively lower amount of ${ }^{13} \mathrm{C}$ that is ingested (partially due to the third ${ }^{13} \mathrm{C}$ burning shell; see Sect. 7.1.1), leading to a smaller quantity of neutrons. However, the last thermal pulses of this star ingest increasing amounts of ${ }^{13} \mathrm{C}$ and ${ }^{14} \mathrm{~N}$ from the inter-shell, leading to an increasing production of ${ }^{14} \mathrm{C}$. Due to its short lifetime $\left(t_{1 / 2}=5730 \mathrm{yr}\right),{ }^{14} \mathrm{C}$ partially decays during the inter-pulse phases of our less massive AGB models. This depletion is however reduced by the ${ }^{14} \mathrm{C}$ production inside the radiative ${ }^{13} \mathrm{C}$ burning shell. For the most massive AGB 
stars that have inter-pulse durations shorter that $t_{1 / 2},{ }^{14} \mathrm{C}$ mostly behaves like a stable nuclide, even if strongly destroyed by $\alpha$ captures. Consequently, as explained with more details in Forestini et al. (1996), intermediate-mass AGB stars are globally not important producers of ${ }^{14} \mathrm{C}$.

Apart from the first two thermal pulses (whatever the initial mass or metallicity), ${ }^{14} \mathrm{~N}$ is almost completely destroyed inside thermal pulses through ${ }^{14} \mathrm{~N}(\alpha, \gamma)$. This reaction, as first noted by Iben (1976), also significantly contributes to the energetics of a thermal pulse. Only a small part of ${ }^{14} \mathrm{~N}$ is destroyed through the ${ }^{14} \mathrm{~N}(\mathrm{n}, \mathrm{p}){ }^{14} \mathrm{C}$ reaction. Indeed, the inter-shell mean mass fraction of ${ }^{14} \mathrm{~N}$ is ranged between $\sim 10^{-2}$ for the $3 M_{\odot}$ star with $Z=0.02$ and $\sim 10^{-3}$ for the $5 M_{\odot}$ star with $Z=0.005$, while the production level of ${ }^{14} \mathrm{C}$ is $\sim 10^{-5}\left(\sim 10^{-6}\right)$, in mass fraction, for the $Z=0.02(0.005)$ models (except for the $3 M_{\odot}$ with $Z=0.02$ star; see above).

As are ${ }^{13} \mathrm{C}$ and ${ }^{14} \mathrm{~N},{ }^{15} \mathrm{~N}$ is engulfed in a thermal pulse from the inter-shell region. The only significant reaction that produces this element inside the convective tongue itself is ${ }^{18} \mathrm{O}(\mathrm{p}, \alpha)$ (see below for the ${ }^{18} \mathrm{O}$ production). On the other hand, ${ }^{15} \mathrm{~N}$ is mainly destroyed by the ${ }^{15} \mathrm{~N}(\alpha, \gamma)$ reaction. Its rate is lower than that of ${ }^{14} \mathrm{~N}(\alpha, \gamma)$ so that ${ }^{15} \mathrm{~N}$ is efficiently destroyed in all the stars only in the asymptotic regime, i.e. when thermal pulses become very intense. Remember that it arrives earlier for lower $Z$ objects. Note that the $3 M_{\odot}$ stars (whatever $Z$ ) maintain a relatively higher ${ }^{15} \mathrm{~N}$ abundance, especially during the full amplitude thermal pulses. Indeed, while these thermal pulses efficiently destroy ${ }^{15} \mathrm{~N}$ whatever the initial mass of the star, its initial abundance inside the convective tongue is higher for $3 M_{\odot}$ objects. This is due to the fact that the engulfed inter-shell region of $3 M_{\odot}$ stars, that contains ${ }^{15} \mathrm{~N}$, is significantly thicker (in mass) compared to that of more massive ones.

${ }^{16} \mathrm{O}$ being partially destroyed in the HBS, the mixing of the inter-shell region reduces its mean mass fraction. On the other hand, at the maximum extent of the thermal pulses, it is enhanced, like ${ }^{12} \mathrm{C}$, due to the downwards penetration of the convective tongue. From pulse to pulse, it also becomes significantly produced by ${ }^{12} \mathrm{C}(\alpha, \gamma)$. This is especially visible for $Z=0.005$ stars, the initial ${ }^{16} \mathrm{O}$ content of which is lower. This primary ${ }^{16} \mathrm{O}$ production by the thermal pulses finally stabilizes around $\sim 10^{-2}$ (in mass fraction). The decrease observed during the last thermal pulses of the $3 M_{\odot}$ AGB star with $Z=0.02$ traduces the more important role of mixing of inter-shell material (already emphasized for ${ }^{14} \mathrm{~N}$ and ${ }^{15} \mathrm{~N}$ ).

The ${ }^{17} \mathrm{O}$ previously contained in the inter-shell region is also mixed in the convective tongue. It is not produced inside thermal pulses. On the contrary, it can be destroyed mainly through ${ }^{17} \mathrm{O}(\alpha, \mathrm{n})$ and ${ }^{17} \mathrm{O}(\alpha, \gamma)$, the first reaction being in general at least ten times faster than the second one. This weakly contributes to produce neutrons inside the thermal pulses. This ${ }^{17} \mathrm{O}$ destruction already operates from the first thermal pulses, whatever the initial
$M$ and $Z$. In later thermal pulses, the ${ }^{17} \mathrm{O}$ mass fraction stabilizes around 4 to $810^{-10}$, that represents a balance between the amount of ${ }^{17} \mathrm{O}$ brought from the inter-shell region and destroyed by the thermal pulse. Again, our $3 M_{\odot}$ models with $Z=0.02$ indicate a still dominant role of the inter-shell mixing. It is less true for the $Z=0.005$ models because at lower $Z$, thermal pulses are somewhat hotter, that favors ${ }^{17} \mathrm{O}$ depletion.

Contrarily to the case of ${ }^{17} \mathrm{O}$, the inter-shell mass fraction of ${ }^{18} \mathrm{O}$ is very low ( $\lesssim 10^{-9}$ typically). Its presence inside thermal pulse consequently results from its production by the ${ }^{14} \mathrm{~N}(\alpha, \gamma){ }^{18} \mathrm{~F}$ reaction. Note that the rapid ${ }^{18} \mathrm{~F} \beta$-decay to ${ }^{18} \mathrm{O}$ also significantly contributes to the thermal pulse energetics. This element is destroyed by three reactions that are, by decreasing order of nuclear time scales, ${ }^{18} \mathrm{O}(\alpha, \gamma),{ }^{18} \mathrm{O}(\alpha, \mathrm{n})$ and ${ }^{18} \mathrm{O}(\mathrm{p}, \alpha){ }^{15} \mathrm{~N}$. This last reaction can even become faster than ${ }^{18} \mathrm{O}(\alpha, \mathrm{n})$ when significant amounts of protons are produced. This especially arrives for the late thermal pulses of the $3 M_{\odot}$ star with $Z=0.02$. The convective tongues being hotter for more massive AGB stars and/or later along the TPAGB phase of a given star, the ${ }^{18} \mathrm{O}$ destruction, which is very sensitive to temperature, becomes more and more efficient from pulse to pulse. This point was already noted by Boothroyd \& Sackmann (1988b). The thermal pulse equilibrium mean mass fraction between production and destruction is ranged between $10^{-7}$ and $10^{-6}$ depending on $M$ and $Z$.

As for ${ }^{18} \mathrm{O},{ }^{19} \mathrm{~F}$ is destroyed in the HBS. It is however produced inside thermal pulses. As first demonstrated by Forestini et al. (1992), the ${ }^{19} \mathrm{~F}$ synthesis involves many nuclear reactions, so that it constitutes a good tracer of the nucleosynthesis conditions prevailing inside thermal pulses. ${ }^{19} \mathrm{~F}$ is produced by the ${ }^{15} \mathrm{~N}(\alpha, \gamma)$ reaction. The rather rapid increase of the mean ${ }^{19} \mathrm{~F}$ mass fraction during the first thermal pulses of each AGB star is due to the increasing importance of the inter-shell ${ }^{15} \mathrm{~N}$ destruction. When convective tongues become hot enough to completely destroy the engulfed ${ }^{15} \mathrm{~N}$, the following thermal pulses only ingest ${ }^{15} \mathrm{~N}$ from matter that has not experienced the preceding thermal pulse (i.e. $\$ 50 \%$ of the convective tongue extension in mass typically; see Table 4). However, as we have explained above, ${ }^{15} \mathrm{~N}$ can be produced by the chain ${ }^{14} \mathrm{~N}(\alpha, \gamma){ }^{18} \mathrm{~F}\left(\beta^{+}\right){ }^{18} \mathrm{O}(\mathrm{p}, \alpha){ }^{15} \mathrm{~N}$. Neutrons are mainly coming from the ${ }^{13} \mathrm{C}(\alpha, \mathrm{n})$ reaction, and to a lower extent, from ${ }^{18} \mathrm{O}(\alpha, \mathrm{n}),{ }^{22} \mathrm{Ne}(\alpha, \mathrm{n})$, ${ }^{21} \mathrm{Ne}(\alpha, \mathrm{n})$ (see below) and ${ }^{17} \mathrm{O}(\alpha, \mathrm{n})$. Protons are coming from $(\mathrm{n}, \mathrm{p})$ reactions, mostly on ${ }^{26} \mathrm{Al}^{\mathrm{g}}$ and ${ }^{14} \mathrm{~N}$ (see below). However, (i) only part of the neutrons are available to produce protons as many (n, $\gamma)$ reactions also occur (see Sect. 7.5) and (ii) a small (\$10\%) part of the protons are also captured by other nuclides than ${ }^{18} \mathrm{O}$ (mainly by $\left.{ }^{15} \mathrm{~N}(\mathrm{p}, \alpha){ }^{12} \mathrm{C}\right)$. The success of this reaction chain mainly depends on the rate of ${ }^{18} \mathrm{O}$ destruction, mainly by $\alpha$ captures (see above). This destruction becomes more efficient from pulse to pulse so that only a small fraction of ${ }^{18} \mathrm{O}$ is 
available for the $(\mathrm{p}, \alpha)$ reaction. On the other hand, when convective tongues become very hot (base temperature $\left.>28010^{6} \mathrm{~K}\right),{ }^{19} \mathrm{~F}$ begins to be destroyed by ${ }^{19} \mathrm{~F}(\alpha, \mathrm{p})$ and ${ }^{19} \mathrm{~F}(\mathrm{n}, \gamma)$ (if significant amounts of neutrons are available). In conclusion, three facts concur to considerably decrease the ${ }^{19} \mathrm{~F}$ production after the first thermal pulses (whatever $M$ and $Z$ ). Indeed, from pulse to pulse and especially for the most massive AGB stars,

- the amount of ${ }^{13} \mathrm{C}$ engulfed in the convective tongue of a thermal pulse decreases (due to the partial destruction of ${ }^{13} \mathrm{C}$ in the inter-shell region during the inter-pulse phase; see Sect. 7.1.1);

- the amount of ${ }^{15} \mathrm{~N}$ ingested from the inter-shell region also decreases;

- the $\alpha$ captures on ${ }^{18} \mathrm{O}$ are more frequent;

- the ${ }^{19} \mathrm{~F}$ destruction becomes significant. This can in particular lead to a partial destruction of the ${ }^{19} \mathrm{~F}$ synthesized in the previous thermal pulses for stars with $M>4 M_{\odot}$.

\subsection{NeNa and MgAl nucleosynthesis}

The mean ${ }^{20} \mathrm{Ne}$ abundance is roughly unchanged in the HBS as well as by the thermal pulse nucleosynthesis. In number, the contribution of ${ }^{17} \mathrm{O}(\alpha, \mathrm{n}){ }^{20} \mathrm{Ne}$ is rather negligible.

${ }^{21} \mathrm{Ne}$ is significantly destroyed in the HBS. Inside the convective tongues of quite hot thermal pulses (base temperatures above $\left.27010^{6} \mathrm{~K}\right)$, it is produced by the small proportion of ${ }^{18} \mathrm{O}$ that is destroyed through the ${ }^{18} \mathrm{O}(\alpha, \mathrm{n})$ reaction. There is also a small contribution by ${ }^{17} \mathrm{O}(\alpha, \gamma)$. Only a very small ${ }^{21} \mathrm{Ne}$ fraction can be destroyed by the ${ }^{21} \mathrm{Ne}(\alpha, \mathrm{n})$ at the end of very hot thermal pulses (base temperatures above $29010^{6} \mathrm{~K}$ ).

${ }^{22} \mathrm{Ne}$ is somewhat produced in the HBS. Its abundance is considerably enhanced inside thermal pulses (by typically a factor of 100) due to its production by ${ }^{18} \mathrm{O}(\alpha, \gamma)$. However, its abundance from pulse to pulse becomes constant (or even slightly decreases inside full amplitudes thermal pulses of the most massive stars and/or those with lower $Z$ ). At their maximum extent, such convective tongues are effectively hot enough (base temperatures $\left.>29010^{6} \mathrm{~K}\right)$ to activate the ${ }^{22} \mathrm{Ne}(\alpha, \gamma){ }^{26} \mathrm{Mg}$ and ${ }^{22} \mathrm{Ne}(\alpha, \mathrm{n}){ }^{25} \mathrm{Mg}$ reactions. However, as noted below, only a small part of the ${ }^{22} \mathrm{Ne}$ nuclides are converted into ${ }^{26} \mathrm{Mg}$ or ${ }^{25} \mathrm{Mg}$, respectively (typically one hundredth). Nevertheless, this consists in a non-negligible neutron source for these thermal pulses, as first noted by Käppeler et al. (1990) and emphasized by Straniero et al. (1995).

Concerning ${ }^{23} \mathrm{Na}$, one has to distinguish between the $3,4,5 M_{\odot}$ with $Z=0.02$ and $3 M_{\odot}$ with $Z=0.005$ stars on the one hand, and the $6 M_{\odot}$ with $Z=0.02,4$ and $5 M_{\odot}$ with $Z=0.005$ stars on the other. Inside the former ones, ${ }^{23} \mathrm{Na}$ is significantly produced at the bottom of the HBS and very slightly produced by the HeBS (during the inter-pulse phase, through the ${ }^{20} \mathrm{Ne}(\alpha, \mathrm{p})$ reac- tion). Furthermore, whatever $M$ and $Z,{ }^{23} \mathrm{Na}$ is almost unchanged by the thermal pulse nucleosynthesis. As a consequence, its mean mass fraction inside the convective tongue does not evolve significantly from pulse to pulse. At the opposite, inside the latter ones, the bottom of the HBS is hot enough to somewhat destroy ${ }^{23} \mathrm{Na}$ while it is produced in its upper part. However, the HeBS substantially produces this nuclide during the inter-pulse phase. Consequently, when the convective tongues of such stars penetrate the inter-shell region, they engulf material that is impoverished in ${ }^{23} \mathrm{Na}$; this explains its mean mass fraction decrease from pulse to pulse.

The ${ }^{24} \mathrm{Mg}$ abundance is almost unchanged by the thermal pulse nucleosynthesis too. Furthermore, the HBS of $3 M_{\odot}$ stars is not hot enough to destroy it significantly. Its mean mass fraction in the convective tongue region of such stars consequently remains almost constant from pulse to pulse. The situation is quite different for more massive stars (whatever $Z$ ) as they destroy ${ }^{24} \mathrm{Mg}$ at the bottom of their HBS. Dilution of inter-shell material by the successive convective tongues thus traduces in a mean mass fraction decrease with time.

Intermediate nuclide of the $\mathrm{MgAl}$ chain, ${ }^{25} \mathrm{Mg}$ is modified by the proton burning inside the HBS. We refer to Sect. 7.1.1 for more details (dependence on $M$ and $Z$ ). However, the evolution of its mean mass fraction inside the convective tongues of thermal pulses is dominated by its production through the ${ }^{22} \mathrm{Ne}(\alpha, \mathrm{n})$ reaction (operating at a quite comparable rate than ${ }^{22} \mathrm{Ne}(\alpha, \gamma)$, following our present knowledge of the corresponding nuclear reaction rates). Indeed, even if this reaction is very slow inside thermal pulses of relatively low mass AGB stars (especially for their first thermal pulses), ${ }^{22} \mathrm{Ne}$ is much more abundant than ${ }^{25} \mathrm{Mg}$ (by typically a factor of one hundred) in that region, that explains the sensible increase of the ${ }^{25} \mathrm{Mg}$ mean mass fraction.

${ }^{26} \mathrm{Mg}$ is slightly (significantly) depleted in the HBS of the $Z=0.02(0.005)$ models. On the other hand, it is substantially produced by the thermal pulse nucleosynthesis (i) by the ${ }^{22} \mathrm{Ne}(\alpha, \gamma)$ reaction (same remark as for ${ }^{25} \mathrm{Mg}$ above) and, to a lower extent, (ii) by the ${ }^{26} \mathrm{Al}^{\mathrm{g}}$ (n, p) reaction (see below). The slope with which its mean mass fraction increases form pulse to pulse is consequently higher for hotter convective tongues, i.e. steeper for full amplitude thermal pulses of massive AGB stars.

${ }^{26} \mathrm{Al}^{\mathrm{g}}$ is one of the most important by-product of the HBS (see above). With a lifetime $t_{1 / 2}=710^{5} \mathrm{yr}$, i.e. much longer than the inter-pulse duration of intermediate-mass AGB stars, it essentially behaves like a stable nuclide in the inter-shell region. When engulfed inside the convective tongue of a thermal pulse, it is mainly destroyed through the ${ }^{26} \mathrm{Al}^{\mathrm{g}}(\mathrm{n}, \mathrm{p})$ reaction and, at least ten times slower, by ${ }^{26} \mathrm{Al}^{\mathrm{g}}(\mathrm{n}, \alpha)$. As a result, ${ }^{26} \mathrm{Al}^{\mathrm{g}}$ is the most important proton source inside thermal pulses, whatever $M$ and $Z$. Its destruction is however partial, due to the relatively low neutron abundance (see Sect. 7.5 below). Note that 
${ }^{26} \mathrm{Al}^{\mathrm{g}}$ (n, p) is also the principal neutron source in the radiative ${ }^{13} \mathrm{C}$ burning shell, as reported by Wasserburg et al. (1994) too.

${ }^{27} \mathrm{Al}$ is significantly produced in the HBS, especially in the most massive AGB stars. It does not significantly participate to the thermal pulse nucleosynthesis. As a consequence, it is engulfed by the convective tongue from the inter-shell region and its corresponding mean mass fraction slightly increases from pulse to pulse. Note however that with time, most of the H-burning of the $5 M_{\odot}$ AGB star with $Z=0.005$ occurs inside its convective envelope. This considerably reduces the ${ }^{27} \mathrm{Al}$ production.

$\mathrm{Si}, \mathrm{P}$ and $\mathrm{S}$ are almost not concerned by charged particle nuclear reactions inside the convective tongue of thermal pulses. On the other hand, they are also rather unchanged in the HBS. The only role played by these elements concerns neutron captures that we now briefly discuss.

\subsection{Neutron production}

Many evolved AGB stars show considerable overabundances in elements heavier than iron (see e.g. Smith \& Lambert 1986). The original discovery by Merrill (1952) of Tc in the spectra of some S stars definitively proved that these heavy elements are synthesized inside AGB stars. Iben (1975) first demonstrated how such species can indeed be produced inside the thermal pulses by slow neutron captures (the so-called s-process), those neutrons coming from the ${ }^{22} \mathrm{Ne}(\alpha, \mathrm{n})$ reaction. However, Iben's computations concerned a very massive AGB star $\left(7 M_{\odot}\right)$, while ever since, all the observed stars showing $s$ elements enrichment have been identified as being of lower mass (i.e. $<6 M_{\odot}$ and for many of them $<3 M_{\odot}$ ). More recent observations (Aaronson \& Mould 1985) and evolutionary models (Malaney \& Boothroyd 1987) then strongly suggested that the major neutron source had to be the ${ }^{13} \mathrm{C}(\alpha, \mathrm{n})$ reaction.

${ }^{13} \mathrm{C}$ is engulfed by the convective tongues of thermal pulses from the inter-shell region where the HBS ashes accumulate. However, the amount of ${ }^{13} \mathrm{C}$ spread out by the HBS is by far (i.e. at least a factor of 10 or more) insufficient to explain the s-process that requires more than one neutron by seed ${ }^{56} \mathrm{Fe}$. This so-called "s-process mystery" (Sackmann \& Boothroyd 1991) is a common failure of all the recent stellar evolution models of TP-AGB stars. It is moreover reinforced by the fact that other lighter nuclides also capture part of the available neutrons (mainly, by increasing order of atomic mass, ${ }^{12} \mathrm{C},{ }^{14} \mathrm{~N},{ }^{17} \mathrm{O},{ }^{19} \mathrm{~F}$, $\left.{ }^{24} \mathrm{Mg},{ }^{25} \mathrm{Mg},{ }^{26} \mathrm{Al}{ }^{\mathrm{g}},{ }^{28} \mathrm{Si},{ }^{29} \mathrm{Si},{ }^{30} \mathrm{Si},{ }^{31} \mathrm{P},{ }^{32} \mathrm{~S},{ }^{33} \mathrm{~S}, \ldots\right)$. One has to note that while the neutron abundance has to be increased to solve the s-process problem, the present computations probably slightly overestimate the abundances of the above mentioned nuclides.

More specifically, in our thermal pulse models, the ratio of the engulfed mass of ${ }^{13} \mathrm{C}$ to the mass of ${ }^{56} \mathrm{Fe}$ in- side the convective tongue at its maximum extent ranges between 0.002 to 0.03 . This upper limit is reached during the asymptotic regime of all our modeled stars and thus appears to be rather independent of the initial mass or metallicity. Inside their first thermal pulses however, $3 M_{\odot}$ stars already produce neutrons (ratio roughly equal to 0.02 ) while at the opposite, the corresponding thermal pulses of $6 M_{\odot}$ stars do not engulf substantial amounts of ${ }^{13} \mathrm{C}$. Furthermore, we noted (see Sect. 7.4) that the ${ }^{22} \mathrm{Ne}$ neutron source operates after the maximum of very intense thermal pulses, at a rate that is growing with the maximum temperature of the convective tongue, i.e. with initial mass and/or pulse number.

Let us stress that such low quantities of ${ }^{13} \mathrm{C}$ ingested by thermal pulses are partly explained by the partial ${ }^{13} \mathrm{C}$ radiative burning, operating at the bottom of the intershell region during the inter-pulse phase (see Sect. 7.1.1). Consequently, it seems clear that the s-process occuring inside TP-AGB stars has two distinct origins (in time and space):

- during the inter-pulse phase, radiative s-process occurs in a radiative zone that is the lower part of the intershell region; the only neutron source is ${ }^{13} \mathrm{C}$;

- during and inside the convective tongue associated with thermal pulses, the classical s-process takes place with two possible neutron sources: (i) the remainder amount of ${ }^{13} \mathrm{C}$ that is engulfed by the convective tongue and, to a lower extent (maximum a few percents), (ii) the ${ }^{22} \mathrm{Ne}$, close to the end of hot thermal pulses. Let us emphasize that the $(\alpha, \mathrm{n})$ and accompanying $(n, \gamma)$ reactions have non-negligible energetic effects on the structural evolution of the convective tongues.

We compute again some thermal pulses by treating together nucleosynthesis and time-dependent convective mixing through Eqs. (4). We observed an abundance gradient inside the convective tongue for a few nuclides that are involved in very fast nuclear reactions (neutrons, protons and some unstable nuclides like ${ }^{18} \mathrm{~F}$ or ${ }^{22} \mathrm{Na}$ ). Globally however, the final abundances resulting from the thermal pulse nucleosynthesis were similar to within $\sim 10 \%$ compared to standard computations. More specifically, neutrons were systematically found to be much more abundant (by typically five orders of magnitude) at the base of the convective tongues, meaning that the ingested ${ }^{13} \mathrm{C}$ is first transported down before it is destroyed by ${ }^{13} \mathrm{C}(\alpha, \mathrm{n})$. The liberated neutrons are very rapidly captured, as attested e.g. by a greater proton abundance at the base of the convective tongue too. This leads us to the conclusion that matter that is actually irradiated by the neutron flux is only the bottom part of each convective tongue. The number of free neutrons by seed ${ }^{56} \mathrm{Fe}$ is so enhanced. Such an approach has already been suggested by Malaney et al. (1988). The conclusion, however, remains that in order to reproduce the observed distribution of $s$ elements in 
the primitive solar system, higher neutron fluxes are still needed.

The most natural way to conciliate observations and theoretical models concerning the production of the $s$ elements should be to increase the amount of ${ }^{13} \mathrm{C}$ in the inter-shell region. This can be done if protons are transported down, e.g. during the $3 \mathrm{DUP} .{ }^{13} \mathrm{C}$ could then be produced by the ${ }^{12} \mathrm{C}(\mathrm{p}, \gamma)$ reaction. Such a scenario would enhance the s-process in both sites where it can occur. In our opinion, the present failure to build this extra-amount of ${ }^{13} \mathrm{C}$ could be related to our bad treatment of convective boundaries, especially when they rapidly penetrates very inhomogeneous regions, like it is the case during the 3DUP events. The possible occurrence of such a slow-particle transport process able to transport protons down into the inter-shell region and its exact efficiency will be quantitatively investigated in the context of low-mass AGB stars. These stars have indeed longer inter-pulse and 3DUP durations that could allow to transport significant amounts of hydrogen downwards (see Sect. 9).

Straniero et al. (1995) have recently shown that such enhanced amounts of ${ }^{13} \mathrm{C}$ in the inter-shell region could indeed allow the radiative s-process to occur. Most interestingly, they found that the resulting signatures are rather similar to those coming from classical s-process computations and are even reached faster. This work is very important as it clearly demonstrates that this so-called ${ }^{~}{ }^{13} \mathrm{C}$ pocket" allows to explain the observed $s$ element synthesis in AGB stars.

\subsection{Resulting abundances of each nuclide inside the various nucleosynthesis sites}

In order to summarize our detailed discussion of nucleosynthesis in the previous sections, we now identify which specific nuclear region, inside a TP-AGB star, mainly contributes to the surface abundance change of each nuclide, when the third dredge-up occurs.

Concerning the light elements, let us just recall (see Sect. 7.2 ) that ${ }^{7} \mathrm{Li}$ can be produced by HBB in the convective envelope of $>4.5 M_{\odot}\left(4 M_{\odot}\right)$ AGB stars with $Z=0.02(0.005)$.

When a third dredge-up occurs, the convective envelope first penetrates the inter-shell region that is depleted in ${ }^{12} \mathrm{C}$ and then reaches a deeper region previously enriched in ${ }^{12} \mathrm{C}$ by the convective tongue. As a result, ${ }^{12} \mathrm{C}$ globally increases in the convective envelope. The situation is reversed for ${ }^{13} \mathrm{C}$. After a 3DUP, the envelope is somewhat impoverished in ${ }^{13} \mathrm{C}$ as this nuclide is almost completely destroyed inside the convective tongue. Consequently, the isotopic ratio ${ }^{12} \mathrm{C} /{ }^{13} \mathrm{C}$ significantly increases at each 3DUP, all the more as the convective envelope mass is reduced. However, in our $\geq 5 M_{\odot}\left(4 M_{\odot}\right) \mathrm{TP}-$ AGB stars with $Z=0.02(0.005)$, HBB is strong enough to partially convert ${ }^{12} \mathrm{C}$ in ${ }^{13} \mathrm{C}$ through the $\mathrm{CN}$ cycle so that the surface ${ }^{12} \mathrm{C} /{ }^{13} \mathrm{C}$ drastically decreases. For these objects, the 3DUP and HBB have opposite effects. Last but not least, ${ }^{14} \mathrm{C}$, somewhat produced by thermal pulses and the third burning shell, is up-heaved to the surface during 3DUP events, where it however substantially decays in the less massive AGB stars.

${ }^{14} \mathrm{~N}$ is produced in the inter-shell region but is strongly destroyed inside thermal pulses. ${ }^{15} \mathrm{~N}$ is destroyed in the inter-shell region while it is somewhat produced by the first few thermal pulses and then destroyed inside full amplitude thermal pulses, i.e. those that can be followed by a 3DUP. As a consequence, the surface isotopic ratio ${ }^{14} \mathrm{~N} /{ }^{15} \mathrm{~N}$ is expected to slightly increase from 3DUP to 3DUP. Again, as the CN cycle operates inside the convective envelope of the most massive AGB stars, ${ }^{14} \mathrm{~N}$ is produced from ${ }^{12} \mathrm{C}$ and ${ }^{15} \mathrm{~N}$ is destroyed, thus leading to very high ${ }^{14} \mathrm{~N} /{ }^{15} \mathrm{~N}$ ratios. If $\mathrm{HBB}$ occurs at very high temperatures (i.e. $\gtrsim 10^{8} \mathrm{~K}$ ) a rather long time enough (i.e. for the lowest mass loss rate), the approach towards the CNO bi-cycle equilibrium leads to an increase of the ${ }^{15} \mathrm{~N}$ abundance and consequently, a decreasing ${ }^{14} \mathrm{~N} /{ }^{15} \mathrm{~N}$ ratio. This mainly concerns our $6 M_{\odot}\left(5 M_{\odot}\right)$ AGB star with $Z=0.02(0.005)$.

${ }^{16} \mathrm{O}$, like ${ }^{12} \mathrm{C}$, is depleted in the inter-shell region, but is produced inside the thermal pulses. During 3DUP events, its surface abundance very slightly increases. ${ }^{17} \mathrm{O}$ is always considerably depleted in both the inter-shell and thermal pulse regions, so that a 3DUP decreases its surface abundance. Finally, at the end of full amplitude thermal pulses, ${ }^{18} \mathrm{O}$ has a lower abundance as inside the convective envelope. As it is also completely destroyed in the inter-shell region, its abundance drastically decreases from 3DUP to 3DUP. Consequently, both ${ }^{16} \mathrm{O} /{ }^{17} \mathrm{O}$ and ${ }^{16} \mathrm{O} /{ }^{18} \mathrm{O}$ surface isotopic ratios significantly increase during each dredgeup. If HBB is strong enough however, the operation of the ON cycle increases the amount of ${ }^{17} \mathrm{O}$ to the expense of ${ }^{16} \mathrm{O}$ and substantially destroys ${ }^{18} \mathrm{O}$. So, in massive AGB stars, ${ }^{16} \mathrm{O} /{ }^{17} \mathrm{O}$ can decrease while ${ }^{16} \mathrm{O} /{ }^{18} \mathrm{O}$ further increases much more than in stars that do not experience HBB.

${ }^{19} \mathrm{~F}$ is destroyed in the HBS but it is significantly produced by thermal pulses. As a consequence, 3DUP events must lead to correlated ${ }^{19} \mathrm{~F}$ and ${ }^{12} \mathrm{C}$ surface enhancements, since both elements are produced in the same region.

As already explained, ${ }^{20} \mathrm{Ne}$ is almost unchanged in the HBS and thermal pulse nucleosynthesis. ${ }^{21} \mathrm{Ne}$ is destroyed inside the inter-shell region but produced inside thermal pulses, this production slightly increasing from pulse to pulse, whatever $M$ or $Z$. Third dredges-up consequently slightly decreases the ${ }^{20} \mathrm{Ne} /{ }^{21} \mathrm{Ne}$ isotopic ratio. In our $6 M_{\odot}\left(5 M_{\odot}\right)$ AGB models with $Z=0.02(0.005)$ however, ${ }^{21} \mathrm{Ne}$ is partially destroyed by HBB. On the other hand, the ${ }^{20} \mathrm{Ne} /{ }^{22} \mathrm{Ne}$ ratio substantially decreases from 3 DUP to $3 D U P$ due to large ${ }^{22} \mathrm{Ne}$ production in the HBS and especially inside the thermal pulses.

During 3DUP events, the surface abundance of ${ }^{23} \mathrm{Na}$ slightly increases for AGB stars with initial masses up to 
$4 M_{\odot}\left(3 M_{\odot}\right)$ and $Z=0.02(0.005)$, due to its production in the HBS. The hotter HBS of more massive AGB stars do not produce ${ }^{23} \mathrm{Na}$, globally. Our $6 M_{\odot}\left(5 M_{\odot}\right)$ models for the same respective $Z$ even slightly destroy ${ }^{23} \mathrm{Na}$ by HBB.

${ }^{24} \mathrm{Mg}$ is somewhat destroyed in the HBS of our most massive AGB stars, especially those with $Z=0.005$, while its abundance does not change significantly after the thermal pulse nucleosynthesis. ${ }^{25} \mathrm{Mg}$ is depleted in the intershell region of our $3 M_{\odot}$ models with $Z=0.02$ as well as in the $Z=0.005$ models of all masses. It is however produced in the HBS of $>4 M_{\odot}$ AGB stars with $Z=0.02$. Nevertheless, its surface abundance evolution following 3DUP events is mainly conditioned by its large abundance (compared to the envelope one) in the region where thermal pulses occur, especially inside the most massive AGB stars. Therefore, the surface ${ }^{24} \mathrm{Mg} /{ }^{25} \mathrm{Mg}$ isotopic ratio necessarily decreases in all AGB stars. The same is true for the ${ }^{24} \mathrm{Mg} /{ }^{26} \mathrm{Mg}$ ratio that roughly behaves in a similar way. Reasons are also rather similar, even if ${ }^{26} \mathrm{Mg}$ is less modified than ${ }^{25} \mathrm{Mg}$ in the HBS. At the surface of our $6 M_{\odot}\left(5 M_{\odot}\right)$ with $Z=0.02(0.005)$ AGB stars, the abundances of both ${ }^{25} \mathrm{Mg}$ and ${ }^{26} \mathrm{Mg}$ can be enhanced to the expense of ${ }^{24} \mathrm{Mg}$, if $\mathrm{HBB}$ is strong enough, leading to very low ${ }^{24} \mathrm{Mg} /{ }^{25} \mathrm{Mg}$ and ${ }^{24} \mathrm{Mg} /{ }^{26} \mathrm{Mg}$ ratios. As we will see in Sect. 8.1 however, this only concerns the end of the TPAGB phase in extreme situations allowing HBB to occur a long time enough (e.g. in case of relatively low mass loss rates).

Only a very small amount of ${ }^{26} \mathrm{Al}^{\mathrm{g}}$ is present in the convective envelope of E-AGB stars as a result of the first (and eventually the second) dredge(s)-up. Along the TPAGB phase, ${ }^{26} \mathrm{Al}^{g}$ is largely produced in the HBS and most of it accumulates in the inter-shell region. Inside full amplitude thermal pulses however, it is somewhat destroyed. For its part, ${ }^{27} \mathrm{Al}$ is slightly produced in the HBS, especially inside the most massive AGB stars. Consequently, ${ }^{26} \mathrm{Al} /{ }^{2}{ }^{27} \mathrm{Al}$ significantly increases with time during the TP-AGB phase, due to the repetitive 3DUP episodes. Of course, dilution by convective mixing being more important for increasing $M$, the highest ratios are expected at the surface of the less massive AGB stars. However, the same extreme cases mentioned in the case of the $\mathrm{Mg}$ isotopic ratios can lead to very high ${ }^{26} \mathrm{Al}^{\mathrm{g}} /{ }^{27} \mathrm{Al}$ ratios for the most massive AGB stars.

From $\mathrm{Si}$, the 3DUP does not change significantly the surface isotopic ratios. However, as stressed in Sect. 7.5, when models will be able to reproduce the $s$ element enhancements at the surface of evolved AGB stars, Si and $\mathrm{S}$ isotopic ratios could be slightly modified by the high neutron flux.

\section{Wind composition along TP-AGB phase}

In this last section, we present our predictions concerning (i) the evolution of all the surface isotopic ratios along the

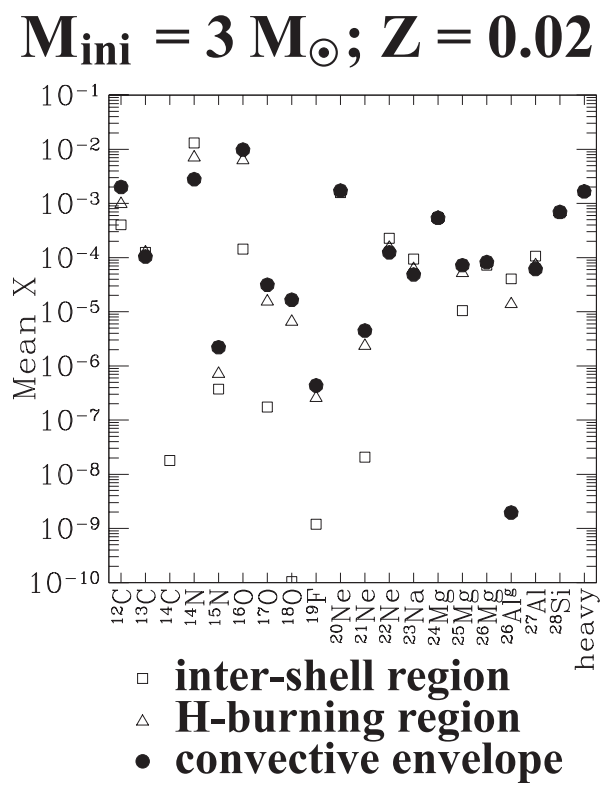

Fig. 7. Mean mass fractions of the 20 most relevant nuclides inside the convective envelope, the HBS and the inter-shell region, for the $3 M_{\odot}(Z=0.02)$ star

TP-AGB phase (by including our evolutionary models and extrapolations up to the convective envelope removal) and (ii) the total amount of material ejected at different ages for all the species up to ${ }^{28} \mathrm{Si}$. We refer to the previous sections for detailed explanations. Thus, in the following, we will just comment these predictions.

\subsection{Surface isotopic ratios along TP-AGB}

Figures 9 and 10 display, for the $3 M_{\odot}$ AGB star with $Z=0.02$, the evolution of the isotopic ratios as a function of the total remaining mass that decreases due to mass loss. Similar figures for the other six AGB stars are only displayed in the electronic version.

Let us stress that we stop our calculations at the last thermal pulse that occurs before the convective envelope mass is reduced below $0.2 M_{\odot}$. This stage indeed roughly corresponds to the PN ejection that we do not maintain to model here. This explains the quite large remaining total stellar masses in the figures, especially for those computed with the highest mass loss rates.

The first comment concerns the formation of carbon stars. $3 M_{\odot}$ AGB stars with $Z=0.02$ and 3 and $4 M_{\odot}$ AGB stars with $Z=0.005$ become carbon stars during their TP-AGB phase. The surface ${ }^{12} \mathrm{C} /{ }^{16} \mathrm{O}$ becomes greater than unity more rapidly for (i) decreasing total mass and/or (ii) decreasing $Z$. This metallicity dependence is confirmed by the increase of $\mathrm{C}$ stars compared to $\mathrm{M}$ stars from the galactic bulge to LMC and SMC (Blanco et al. 1978). Our $6 M_{\odot}\left(5 M_{\odot}\right)$ AGB stars with $Z=0.02$ (0.005) can also become $\mathrm{C}$ stars with our lowest mass loss rates. In such cases indeed, in spite of very efficient HBB 


\section{$\mathrm{M}_{\mathrm{ini}}=\mathbf{3} \mathrm{M}_{\odot} ; \mathrm{Z}=\mathbf{0 . 0 2}$}
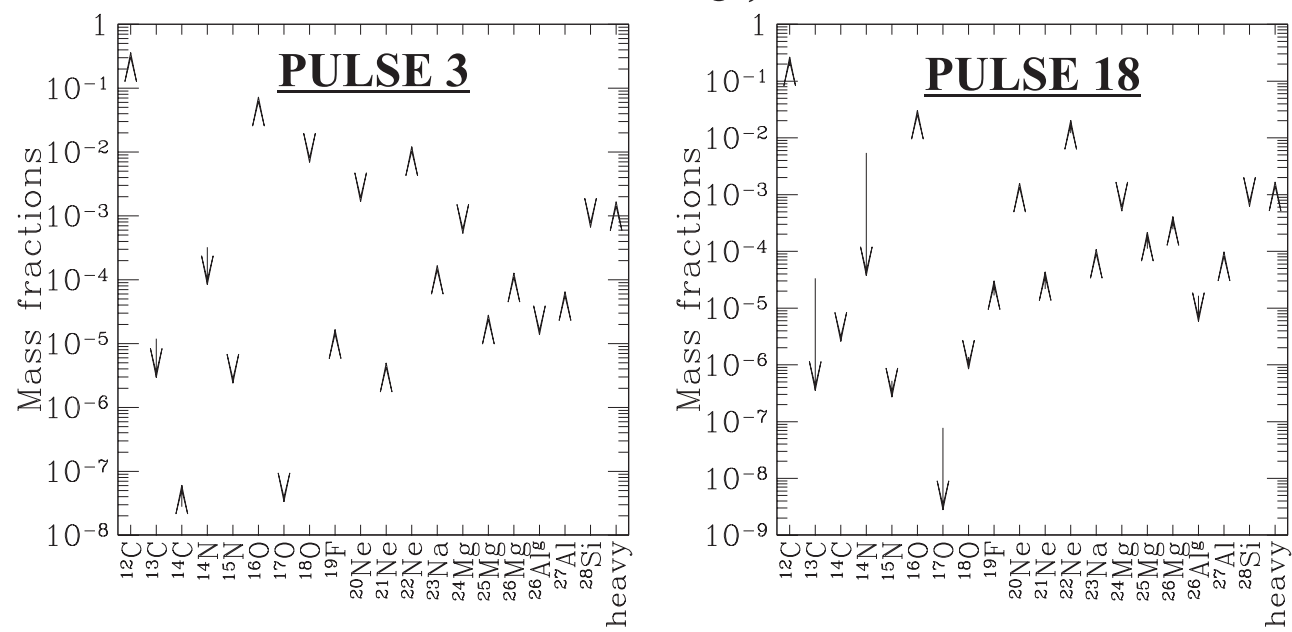

Fig. 8. Variation of the mean mass fractions of the same 20 nuclides as in Fig. 7, due to the thermal pulse nucleosynthesis, for the $3 M_{\odot}(Z=0.02)$ star, inside an early thermal pulse (the $\left.3 \mathrm{~d}\right)$ and an asymptotic one (the 18th). Arrows indicate the mean abundance evolution from the beginning of a thermal pulse to its end

transforming ${ }^{12} \mathrm{C}$ in ${ }^{13} \mathrm{C}$ and ${ }^{14} \mathrm{~N}$, many $3 \mathrm{DUP}$ events can still occur with very reduced envelope masses, so that the relatively short inter-pulse phases are not long enough to substantially destroy ${ }^{12} \mathrm{C}$. We will see however, together with other isotopic ratios, that these very low mass loss rates seem actually extreme. Let us emphasize that the only super-lithium-rich star (see Sect. 7.2) we found to be also a carbon star is the $5 M_{\odot}$ with $Z=0.005$ one (with our medium mass loss rate). However, as already mentioned in Sect. 6.2, observations indicate that $Z=0.02$ carbon stars are formed easier (i.e. at lower luminosities) than predicted by all the models. Let us also stress the very high ${ }^{12} \mathrm{C} /{ }^{13} \mathrm{C}$ isotopic ratios that can be expected at the surface of $\leq 4 M_{\odot}$ AGB stars, whatever $Z$. On the other hand, more massive AGB stars experience $\mathrm{HBB}$ that progressively leads the ${ }^{12} \mathrm{C} /{ }^{13} \mathrm{C}$ isotopic ratio close to its $\mathrm{CN}$ cycle equilibrium value (formation of $\mathrm{J}$ stars). The case of $4 M_{\odot}$ stars with $Z=0.005$ very clearly shows the competition between the 3DUP episodes (increasing ${ }^{12} \mathrm{C} /{ }^{13} \mathrm{C}$ ) and HBB (decreasing it). For lower mass loss rates, the 3DUP events are more numerous for the same total mass decrease, leading to a more rapid ${ }^{12} \mathrm{C} /{ }^{13} \mathrm{C}$ increase. However, the corresponding longer TP-AGB phase allows the base of the convective envelope to reach higher temperatures that finally destroy ${ }^{12} \mathrm{C}$ through the $\mathrm{CN}$ cycle. This efficient transformation of ${ }^{12} \mathrm{C}$ to ${ }^{13} \mathrm{C}$ also reduces the ${ }^{12} \mathrm{C} /{ }^{16} \mathrm{O}$ ratio, i.e. the possibility to form carbon stars. It happens if the temperatures at the base of the convective envelopes become higher than $\sim 7510^{6} \mathrm{~K}$. Such stars have corresponding surface luminosities $>25000 L_{\odot}$ $\left(30000 L_{\odot}\right)$ for $Z=0.02(0.005)$, i.e. $M_{\text {bol }}<-6.25$ $(-6.45)$. We consequently predict that $\mathrm{C}$ stars cannot be formed at higher luminosities than this threshold, due to strong HBB. This is in agreement with Boothroyd et al. (1993) predictions. This also corresponds to the observed threshold for AGB stars of the LMC (Cohen et al. 1981).

Finally, note the surface pollution in ${ }^{14} \mathrm{C}$ that is more important, compared to that of ${ }^{13} \mathrm{C}$, for decreasing total masses. For $3 M_{\odot}$ AGB stars, the surface ${ }^{13} \mathrm{C} /{ }^{14} \mathrm{C}$ ratio is quite independent of the mass loss rate. This is due to the inter-pulse duration that is significantly greater than the ${ }^{14} \mathrm{C}$ lifetime, so that the ${ }^{14} \mathrm{C}$ decay is the dominant factor for the ${ }^{13} \mathrm{C} /{ }^{14} \mathrm{C}$ ratio evolution at the surface of such stars. The quite complicated behavior of the ${ }^{13} \mathrm{C} /{ }^{14} \mathrm{C}$ isotopic ratio at the surface of our most massive models is again due to the competition between the 3DUP that adds ${ }^{14} \mathrm{C}$ into the convective envelope and HBB that can partially destroy it. By extrapolation, it is reasonable to expect even lower ${ }^{13} \mathrm{C} /{ }^{14} \mathrm{C}$ ratios in low-mass AGB stars. As mentioned in Forestini et al. (1996), ${ }^{14} \mathrm{C}$ could become detectable for $<3 M_{\odot}$ evolved AGB stars.

Due to HBB, those stars showing very low surface ${ }^{12} \mathrm{C} /{ }^{13} \mathrm{C}$ ratios are also expected to have very high ${ }^{14} \mathrm{~N} /{ }^{15} \mathrm{~N}$ ones. It has to be stressed indeed that in our most massive AGB stars, the CN cycle operating inside the convective envelope produces large amounts of ${ }^{14} \mathrm{~N}$.

The ${ }^{16} \mathrm{O} /{ }^{17} \mathrm{O}$ and ${ }^{16} \mathrm{O} /{ }^{18} \mathrm{O}$ surface isotopic ratios also evolve as expected from our discussions in Sect. 7. Note that quite low ${ }^{16} \mathrm{O} /{ }^{17} \mathrm{O}$ and very high ${ }^{16} \mathrm{O} /{ }^{18} \mathrm{O}$ ratios, which are clear signatures of the operation of the $\mathrm{ON}$ cycle at the base of the convective envelope (HBB), appear rather simultaneously with the $\mathrm{CN}$ cycle signatures in our most massive objects. Also remark the progressive ${ }^{16} \mathrm{O} /{ }^{18} \mathrm{O}$ decrease in our $6 M_{\odot}\left(5 M_{\odot}\right)$ AGB star with $Z=0.02(0.005)$. For these objects, the temperature at 
the base of the convective envelope finally becomes high enough for ${ }^{16} \mathrm{O}$ to partially burn.

The ${ }^{19} \mathrm{~F}$ surface enhancements we predict are displayed in a special form, corresponding to the observation presentations adopted by Jorissen et al. (1992). Compared to their Fig. 8, two conclusions can be stated.

- There is a clear observed correlation between the ${ }^{19} \mathrm{~F}$ excess and the degree of ${ }^{12} \mathrm{C}$ surface pollution, that is perfectly understandable from the identified nucleosynthetic processes and the mixing episodes (see Sect. 7). Furthermore, our models clearly indicate a trend: the less massive the AGB star, the more efficient the ${ }^{19} \mathrm{~F}$ surface enhancement. In fact, our most massive models even show a surface ${ }^{19} \mathrm{~F}$ depletion due to its partial destruction by HBB.

- Our $Z=0.02$ models well reproduce only the less ${ }^{19} \mathrm{~F}$ enriched stars in the $\left[{ }^{19} \mathrm{~F} /{ }^{16} \mathrm{O}\right]$ versus ${ }^{12} \mathrm{C} /{ }^{16} \mathrm{O}$ diagram. Based on the trend just mentioned, we predict that the AGB stars showing very large ${ }^{19} \mathrm{~F}$ surface enhancements have initial total masses $<3 M_{\odot}$. We plan to verify this statement, i.e. that low-mass AGB stars are the most efficient ${ }^{19} \mathrm{~F}$ galactic producers (see Sect. 9).

As expected, the ${ }^{24} \mathrm{Mg} /{ }^{25} \mathrm{Mg}$ and ${ }^{24} \mathrm{Mg} /{ }^{26} \mathrm{Mg}$ surface isotopic ratios are much less sensitive to the 3DUP episodes and HBB. More precisely, only our most massive models with the lowest mass loss rates show large ${ }^{24} \mathrm{Mg}$ depletions due to a very strong HBB. Again, these are however quite extreme situations.

Last but not least, we predict substantial ${ }^{26} \mathrm{Al}^{\mathrm{g}}$ surface enhancements due to the repetitive dredges-up of the HBS and inter-shell regions. The ${ }^{26} \mathrm{Al}^{\mathrm{g}} /{ }^{27} \mathrm{Al}$ isotopic ratio tends to decrease with increasing total mass due to (i) the greater dilution as mixing occurs in a more massive convective envelope and (ii) the thinner HBS and inter-shell regions (in mass). Nevertheless, ${ }^{26} \mathrm{Al}^{\mathrm{g}} /{ }^{27} \mathrm{Al}$ could well be increasing again if ${ }^{26} \mathrm{Al}^{\mathrm{g}}$ was produced inside the convective envelope itself by strong HBB. Note however that such a very high ${ }^{26} \mathrm{Al}^{\mathrm{g}}$ production level is actually expected to be accompanied by very low $\mathrm{Mg}$ isotopic ratios, as ${ }^{26} \mathrm{Al}^{\mathrm{g}}$ is produced by the $\mathrm{MgAl}$ chain in the extreme situations mentioned above. Let us finally mention that Wasserburg et al. (1994) presented computations in which the evolution of the ${ }^{26} \mathrm{Al}^{\mathrm{g}}$ surface abundance has been followed self-consistently, by including the effect of neutron irradiation due to an enhanced amount of ${ }^{13} \mathrm{C}$ in the inter-shell region (to engender a s-process).

Our results compare very well with those obtained by Boothroyd et al. $(1994,1995)$ concerning the O isotopic ratios in intermediate-mass stars. They also mention the problem to reproduce the ${ }^{16} \mathrm{O} /{ }^{17} \mathrm{O}$ ratio, especially in lowmass carbon AGB stars. We cannot go further in this comparison without having yet computed the TP-AGB phase of low-mass stars. Boothroyd et al. (1995) already suggested that to conciliate all the data with observations of low-mass stars, one probably has to take into account of slow-particle transports inside radiative zones, what they called "cool-bottom precessing". That is what we plan to do (see Sect. 9).

\subsection{Other comparisons with observations}

Evolutionary models of TP-AGB stars can be strongly constrained by the confrontation between predicted and observed surface isotopic ratios, especially if different ratios are observed for the same stars. For most of the observed AGB stars, only the $\mathrm{C}$ and $\mathrm{O}$ isotopic ratios have been determined (see e.g. Harris \& Lambert 1984; Harris et al. 1985; Lambert et al. 1986; Harris et al. 1987; Harris \& Lambert 1987; Kahane et al. 1992). Globally, we found agreements. General features come out.

- As the temperatures required at the base of the convective envelope to allow ${ }^{7} \mathrm{Li}$ production (see Sect. 7.2) are significantly lower than those allowing efficient ${ }^{12} \mathrm{C}$ destruction by the $\mathrm{CN}$ cycle, we predict that luminous $\mathrm{C}$ stars enriched in ${ }^{7} \mathrm{Li}$ can exist, especially for lower $Z$ objects (that have lower ${ }^{16} \mathrm{O}$ content). There are indeed a few $\%$ of the observed $\mathrm{C}$ stars that are also super-lithium-rich and there are more numerous in the LMC compared to our galaxy (see e.g. Smith \& Lambert 1990b for the LMC and Feast 1974 for the Galaxy).

- There is no observed $\mathrm{C}$ star with ${ }^{12} \mathrm{C} /{ }^{13} \mathrm{C}<15$. Most of the $\mathrm{J}$ stars show ${ }^{12} \mathrm{C} /{ }^{13} \mathrm{C}$ ratios between 3 et 4 , i.e. very close to the $\mathrm{CN}$ cycle equilibrium value.

- J stars also show rather low ${ }^{16} \mathrm{O} /{ }^{17} \mathrm{O}$ isotopic ratios while only upper limits have been found for the ${ }^{16} \mathrm{O} /{ }^{18} \mathrm{O}$ ratio. Most of the other AGB stars present ${ }^{16} \mathrm{O} /{ }^{17} \mathrm{O}$ ratios ranged between 300 and 4000 and ${ }^{16} \mathrm{O} /{ }^{18} \mathrm{O}$ ratios ranged between 500 and 5000. Only stars with rather high ${ }^{16} \mathrm{O} /{ }^{17} \mathrm{O}$ ratios are difficult to explain with our intermediate-mass AGB models (see also Boothroyd et al. 1995). Also note that the corresponding $\mathrm{O}$ isotopic ratios in the ISM are ${ }^{16} \mathrm{O} /{ }^{17} \mathrm{O}$ $=2460_{-750}^{+750}$ and ${ }^{16} \mathrm{O} /{ }^{18} \mathrm{O}=675_{-200}^{+200}$. Again, there is a problem with ${ }^{17} \mathrm{O}$. Let us however stress that important uncertainties remain concerning the nuclear cross sections of proton captures on ${ }^{17} \mathrm{O}$. On the other hand, low-mass AGB stars are present among those observations.

Presently, there is only one star for which all the isotopic ratios have been determined. It is $\mathrm{Cw}$ Leo. This $\mathrm{C}$ star is one of the nearest evolved AGB star ever observed. Its distance is estimated to $d=150 \pm 50 \mathrm{pc}$ (see e.g. Claussen et al. 1987; Keady et al. 1988; Griffin 1990). This corresponds to a surface luminosity $L \simeq 20000 \pm$ $5000 L_{\odot}$ and a present mass loss rate of roughly $(2.5 \pm 1.0)$ $10^{-5} M_{\odot} \mathrm{yr}^{-1}$. Such a high wind makes it quite optically thick. A very extended circumstellar envelope has been detected to surround $\mathrm{Cw}$ Leo, IRC+10216. IRC+10216 shows evidences for a bi-polar structure (see e.g. Guélin 


\section{$\mathrm{M}_{\mathrm{ini}}=\mathbf{3} \mathrm{M}_{\odot} ; \mathrm{Z}=\mathbf{0 . 0 2}$}
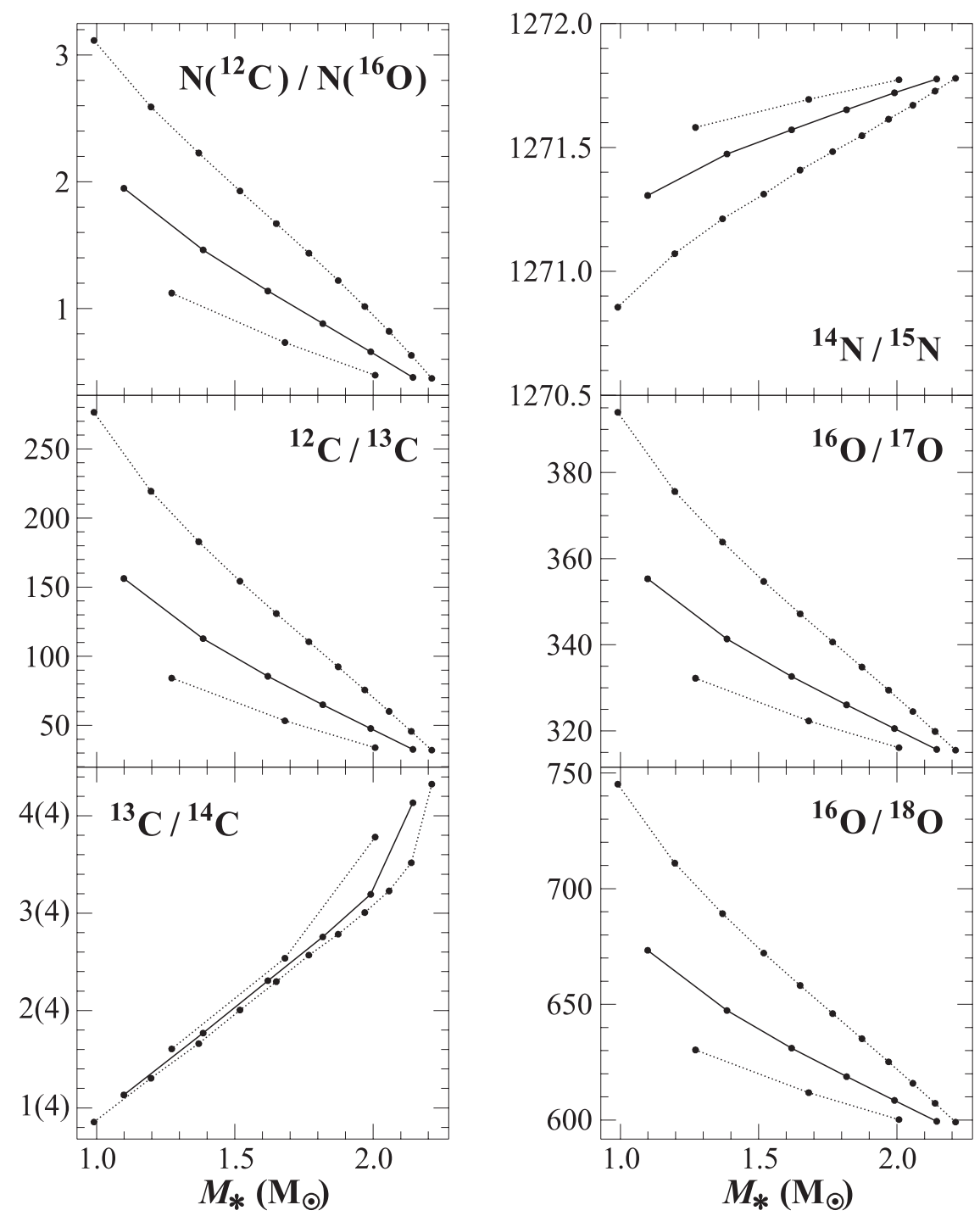

Fig. 9. Surface isotopic ratios as a function of the remaining total stellar mass, that decreases due to the mass loss along the TP-AGB. The solid line refers to the standard mass loss rate (see Sect. 6.3.2 for the corresponding $\eta$ parameter values in the Reimers relation). Dotted lines correspond to mass loss rates increased or decreased by a factor of two. The extrapolated thermal pulses (and 3DUP episodes) are indicated by the filled circles, and are of course more numerous for lower mass loss rates. Here are indicated the ${ }^{12} \mathrm{C} /{ }^{16} \mathrm{O},{ }^{12} \mathrm{C} /{ }^{13} \mathrm{C},{ }^{13} \mathrm{C} /{ }^{14} \mathrm{C},{ }^{14} \mathrm{~N} /{ }^{15} \mathrm{~N},{ }^{16} \mathrm{O} /{ }^{17} \mathrm{O}$ and ${ }^{16} \mathrm{O} /{ }^{18} \mathrm{O}$ ratios, in the case of the $3 M_{\odot}(Z=0.02)$ star. All ratios are in mass fractions, except for the ${ }^{12} \mathrm{C} /{ }^{16} \mathrm{O}$ ratio (given in number abundances)

et al. 1993), attesting that $\mathrm{Cw}$ Leo is very evolved and close to the end of its TP-AGB phase. Consequently, its present convective envelope mass is probably very reduced ( $\lesssim 0.5 M_{\odot}$ typically).

The circumstellar envelope of $\mathrm{Cw}$ Leo allowed very accurate determinations of the isotopic ratios (see Kahane et al. 1988 and 1992; Guélin et al. 1995; Forestini et al. 1996): ${ }^{12} \mathrm{C} /{ }^{13} \mathrm{C}=44+3,3,{ }^{13} \mathrm{C} /{ }^{14} \mathrm{C}>1400,{ }^{14} \mathrm{~N} /{ }^{15} \mathrm{~N}>5300$, ${ }^{16} \mathrm{O} /{ }^{17} \mathrm{O}=840_{-170}^{+230},{ }^{16} \mathrm{O} /{ }^{18} \mathrm{O}=1260_{-240}^{+315},{ }^{24} \mathrm{Mg} /{ }^{25} \mathrm{Mg}$ $=7.6-1.1,{ }^{24} \mathrm{Mg} /{ }^{26} \mathrm{Mg}=6.5_{-0.7}^{+0.7}$ and ${ }^{26} \mathrm{Al}{ }^{\mathrm{g}} /{ }^{27} \mathrm{Al} \gtrsim 0.01$ (to be confirmed). Furthermore, the Si isotopic ratios appear to have interstellar medium values, i.e. unaltered by the star. As derived in Guélin et al. (1995) and Forestini et al. (1996), almost all these isotopic ratios seem to be consistent (within the error bars), following our predictions, with an AGB star that had a main sequence total mass between 4 and $5 M_{\odot}$. However, ${ }^{16} \mathrm{O} /{ }^{17} \mathrm{O}$ is again not well explained. As various surface isotopic ratios (especially ${ }^{14} \mathrm{~N} /{ }^{15} \mathrm{~N}$ and ${ }^{16} \mathrm{O} /{ }^{18} \mathrm{O}$ ) are significantly changing between 4 and $5 M_{\odot}$ AGB models, we are presently 


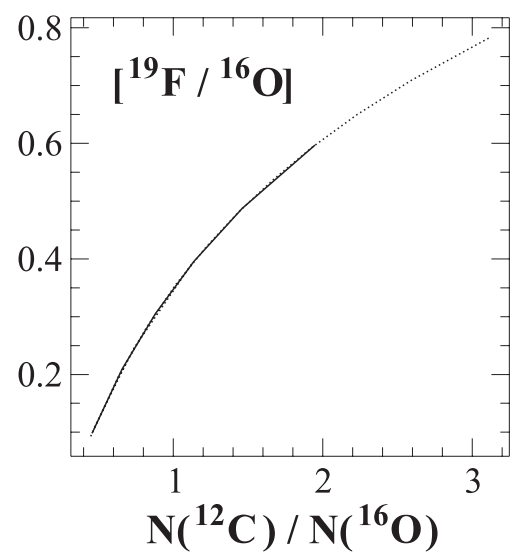

\section{$M_{\text {ini }}=3 M_{\odot} ; Z=0.02$}

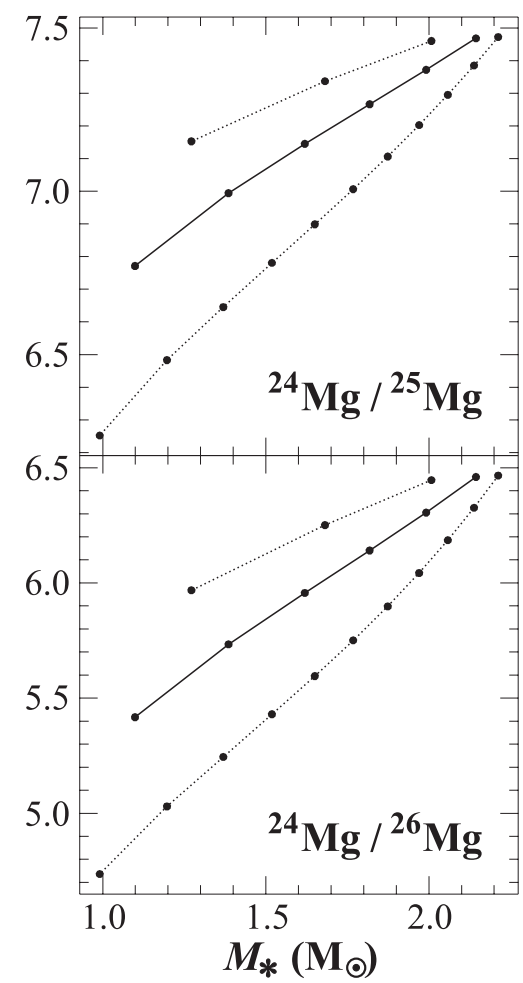

Fig. 10. ${ }^{24} \mathrm{Mg} /{ }^{25} \mathrm{Mg},{ }^{24} \mathrm{Mg} /{ }^{26} \mathrm{Mg},{ }^{26} \mathrm{Al} / \mathrm{g} /{ }^{27} \mathrm{Al}$ ratios as well as $\left[{ }^{19} \mathrm{~F} /{ }^{16} \mathrm{O}\right]$ as a function of ${ }^{12} \mathrm{C} /{ }^{16} \mathrm{O}$, in the case of the $3 M_{\odot}$ $(Z=0.02)$ star. All ratios are in mass fractions and $[\mathrm{A} / \mathrm{B}]$ means $\log (\mathrm{A} / \mathrm{B})_{*}-\log (\mathrm{A} / \mathrm{B})_{\odot}$

computing the TP-AGB phase of a $4.5 M_{\odot}$ star with $Z=0.02$ in order to improve this last prediction.

Finally, another very interesting way to constraint stellar evolution models of AGB stars is to compare the predicted surface isotopic ratios with those determined in primitive meteorites (mainly through grains included inside carbon rich chondrites). Most of the ${ }^{12} \mathrm{C} /{ }^{13} \mathrm{C},{ }^{14} \mathrm{~N} /{ }^{15} \mathrm{~N}$ and ${ }^{26} \mathrm{Al}{ }^{\mathrm{g}} /{ }^{27} \mathrm{Al}$ isotopic anomalies measured in grains (see e.g. Ott 1991; Zinner et al. 1991a) are consistent with those coming from the surface of evolved TP-AGB stars of intermediate-mass. This is of course in favor of the presence, when the solar system has been formed, of one or a few AGB stars in its surroundings. Such grains are indeed well known to be formed in the cool atmosphere of evolved AGB stars (i.e. when mass loss rates become rather high). Concerning the measured $\mathrm{O}$ isotopic ratios, the situation is not so clear as many grains have ratios significantly out of the corresponding predicted ranges. Some of these grains at least can have been formed in low-mass AGB stars. Again, as mentioned in Sect. 8.1, Boothroyd et al. (1995) recently suggested that in such stars, coolbottom processing can help to reduce these discrepancies. On the contrary, some other grains perfectly agree with our predictions. This is for example the case of a $\sim 3 \mu \mathrm{m}$
$\mathrm{Al}_{2} \mathrm{O}_{3}$ grain of the Bishunpur LL3.1 chondrite by Huss et al. (1994). The derived ratios, ${ }^{16} \mathrm{O} /{ }^{17} \mathrm{O}=385 \pm 5$, ${ }^{16} \mathrm{O} /{ }^{18} \mathrm{O}=853 \pm 30$ and ${ }^{26} \mathrm{Al}{ }^{\mathrm{g}} /{ }^{27} \mathrm{Al}=(1.7 \pm 0.2) 10^{-3}$, are in perfect agreement with ratios corresponding to a $3-$ $4 M_{\odot}$ AGB star of nearly solar metallicity. Finally, other $\mathrm{SiC}^{26} \mathrm{Al}^{\mathrm{g}}$-rich grains raise problems, like that discovered by Zinner et al. (1991b), exhibiting a very high ${ }^{26} \mathrm{Al}{ }^{\mathrm{g}} /{ }^{27} \mathrm{Al}$ ratio of $\simeq 0.23$. It seems hard to explain such a value from our models. However, this grain also exhibits an anomalously large ${ }^{44} \mathrm{Ca} /{ }^{40} \mathrm{Ca}$ isotopic ratio that seems to exclude its AGB star origin.

\subsection{Yields}

Tables 8 to 10 give the total mass ejected by the wind (in $M_{\odot}$ ) for various elements (i) at the top of the first RGB, (ii) at the beginning of the TP-AGB phase and (iii) at the end of the TP-AGB phase (thus including our extrapolated nucleosynthesis calculations). As such data mainly concerns the chemical evolution of galaxies, we also present the net yield (positive or negative) for each element. The data of Tables 8 to 10 correspond to the medium mass loss rates (see Sect. 8.1), i.e. the solid lines of Figs. 9 and 10. Brief comments are made about these predictions in the electronic version. 
Table 8. Total mass ejected (in $M_{\odot}$ ) in the wind for some nuclides at the RGB top, at the end of the E-AGB phase and at the AGB tip, just before the ejection of the planetary nebula. Also given is the net contribution for these elements, i.e. the total final mass ejected reduced by the amount of matter that would have been ejected with the initial composition. Here are presented elements from $\mathrm{He}$ to $\mathrm{N}$

\begin{tabular}{|c|c|c|c|c|c|c|c|c|}
\hline & & \multicolumn{4}{|c|}{$Z=0.02$} & \multicolumn{3}{|c|}{$Z=0.005$} \\
\hline & & $3 M_{\odot}$ & $4 M_{\odot}$ & $5 M_{\odot}$ & $6 M_{\odot}$ & $3 M_{\odot}$ & $4 M_{\odot}$ & $5 M_{\odot}$ \\
\hline$M\left(M_{\odot}\right)$ & $\begin{array}{l}\text { at } R G \text { top } \\
\text { at } t_{0} \\
\text { at } A G B \text { tip }\end{array}$ & $\begin{array}{l}2.9978 \\
2.9151 \\
1.0989\end{array}$ & $\begin{array}{l}3.9985 \\
3.7732 \\
1.0143\end{array}$ & $\begin{array}{l}4.9970 \\
4.7009 \\
1.0750\end{array}$ & $\begin{array}{l}5.9980 \\
5.7167 \\
1.3735\end{array}$ & $\begin{array}{l}2.9996 \\
2.8053 \\
0.9471\end{array}$ & $\begin{array}{l}3.9999 \\
3.5067 \\
1.2519\end{array}$ & $\begin{array}{l}4.9997 \\
4.3149 \\
1.3818\end{array}$ \\
\hline${ }^{3} \mathrm{He}$ & $\begin{array}{l}\text { at } R G \text { top } \\
\text { at } t_{0} \\
\text { at } A G B \text { tip } \\
\text { net yield }\end{array}$ & $\begin{array}{l}2.5910^{-7} \\
1.3010^{-5} \\
2.8710^{-4} \\
2.2510^{-4}\end{array}$ & $\begin{array}{l}1.5110^{-7} \\
2.3510^{-5} \\
3.0310^{-4} \\
2.0710^{-4}\end{array}$ & $\begin{array}{l}2.9310^{-7} \\
2.4110^{-5} \\
1.5710^{-4} \\
3.4610^{-5}\end{array}$ & $\begin{array}{c}1.5910^{-7} \\
1.9610^{-5} \\
5.0010^{-5} \\
-9.4410^{-5}\end{array}$ & $\begin{array}{l}7.7310^{-8} \\
3.2610^{-5} \\
3.3010^{-4} \\
2.7410^{-4}\end{array}$ & $\begin{array}{l}9.7010^{-9} \\
5.7010^{-5} \\
3.0410^{-4} \\
2.2910^{-4}\end{array}$ & $\begin{array}{c}3.5410^{-8} \\
6.5210^{-5} \\
9.1910^{-5} \\
-4.2710^{-6}\end{array}$ \\
\hline${ }^{4} \mathrm{He}$ & $\begin{array}{l}\text { at } R G \text { top } \\
\text { at } t_{0} \\
\text { at } A G B \text { tip } \\
\text { net yield }\end{array}$ & $\begin{array}{c}6.5310^{-4} \\
2.5910^{-2} \\
0.59 \\
3.7610^{-2}\end{array}$ & $\begin{array}{c}4.2810^{-4} \\
6.9110^{-2} \\
0.91 \\
3.2010^{-2}\end{array}$ & $\begin{array}{c}8.6910^{-4} \\
9.5910^{-2} \\
1.32 \\
0.17\end{array}$ & $\begin{array}{c}5.8410^{-4} \\
9.1310^{-2} \\
1.59 \\
0.23\end{array}$ & $\begin{array}{c}1.1510^{-4} \\
5.1110^{-2} \\
0.55 \\
3.5610^{-2}\end{array}$ & $\begin{array}{c}1.8910^{-5} \\
0.13 \\
0.72 \\
3.7010^{-2}\end{array}$ & $\begin{array}{c}8.1010^{-5} \\
0.18 \\
1.28 \\
0.37\end{array}$ \\
\hline${ }^{7} \mathrm{Li}$ & $\begin{array}{l}\text { at } R G \text { top } \\
\text { at } t_{0} \\
\text { at } A G B \text { tip } \\
\text { net yield }\end{array}$ & $\begin{array}{l}1.5910^{-11} \\
2.6310^{-11} \\
2.9210^{-10} \\
-1.8510^{-8}\end{array}$ & $\begin{array}{l}1.0210^{-11} \\
3.3510^{-11} \\
6.3510^{-11} \\
-2.9510^{-8}\end{array}$ & $\begin{array}{c}2.4910^{-11} \\
5.1810^{-11} \\
7.3310^{-8} \\
3.4410^{-8}\end{array}$ & $\begin{array}{c}1.4510^{-11} \\
3.8710^{-11} \\
1.0810^{-7} \\
6.2310^{-8}\end{array}$ & $\begin{array}{l}1.4210^{-14} \\
4.4710^{-12} \\
8.2310^{-11} \\
-5.0010^{-9}\end{array}$ & $\begin{array}{l}2.6710^{-15} \\
1.1510^{-11} \\
4.6010^{-10} \\
-6.3410^{-9}\end{array}$ & $\begin{array}{l}1.2610^{-14} \\
1.9610^{-11} \\
3.5710^{-11} \\
-8.9210^{-9}\end{array}$ \\
\hline${ }^{12} \mathrm{C}$ & $\begin{array}{l}\text { at } R G \text { top } \\
\text { at } t_{0} \\
\text { at } A G B \text { tip } \\
\text { net yield }\end{array}$ & $\begin{array}{l}6.4410^{-6} \\
1.7210^{-4} \\
1.2610^{-2} \\
6.5310^{-3}\end{array}$ & $\begin{array}{l}4.2510^{-6} \\
4.5310^{-4} \\
9.7110^{-3} \\
1.3310^{-4}\end{array}$ & $\begin{array}{c}9.1110^{-6} \\
5.9010^{-4} \\
5.9710^{-3} \\
-6.6210^{-3}\end{array}$ & $\begin{array}{c}5.8410^{-6} \\
5.5610^{-4} \\
2.7410^{-3} \\
-1.2110^{-2}\end{array}$ & $\begin{array}{l}2.1410^{-7} \\
9.0110^{-5} \\
1.0610^{-2} \\
9.0010^{-3}\end{array}$ & $\begin{array}{l}4.0110^{-8} \\
2.3410^{-4} \\
4.4010^{-3} \\
2.1910^{-3}\end{array}$ & $\begin{array}{c}1.9010^{-7} \\
3.4310^{-4} \\
9.6810^{-4} \\
-1.9310^{-3}\end{array}$ \\
\hline${ }^{13} \mathrm{C}$ & $\begin{array}{l}\text { at } R G \text { top } \\
\text { at } t_{0} \\
\text { at } A G B \text { tip } \\
\text { net yield }\end{array}$ & $\begin{array}{l}1.2610^{-7} \\
8.7510^{-6} \\
1.9510^{-4} \\
1.2210^{-4}\end{array}$ & $\begin{array}{l}8.5210^{-8} \\
2.4110^{-5} \\
3.1210^{-4} \\
1.9710^{-4}\end{array}$ & $\begin{array}{l}1.4810^{-7} \\
3.2310^{-5} \\
1.0010^{-3} \\
8.5010^{-4}\end{array}$ & $\begin{array}{l}1.1410^{-7} \\
3.0910^{-5} \\
6.6710^{-4} \\
4.8910^{-4}\end{array}$ & $\begin{array}{l}1.1410^{-8} \\
4.9010^{-6} \\
5.0510^{-5} \\
3.0610^{-5}\end{array}$ & $\begin{array}{l}2.1710^{-9} \\
1.3110^{-5} \\
9.0910^{-5} \\
6.4310^{-5}\end{array}$ & $\begin{array}{l}1.0410^{-8} \\
1.9110^{-5} \\
1.9310^{-4} \\
1.5810^{-4}\end{array}$ \\
\hline${ }^{14} \mathrm{C}$ & $\begin{array}{l}\text { at } R G \text { top } \\
\text { at } t_{0} \\
\text { at } A G B \text { tip } \\
\text { net yield }\end{array}$ & $\begin{array}{c}4.8910^{-30} \\
6.9210^{-26} \\
6.2010^{-9} \\
6.2010^{-9}\end{array}$ & $\begin{array}{c}4.7710^{-29} \\
4.7710^{-29} \\
8.9310^{-9} \\
8.9310^{-9}\end{array}$ & $\begin{array}{l}3.2210^{-21} \\
3.6210^{-21} \\
2.9010^{-9} \\
2.9010^{-9}\end{array}$ & $\begin{array}{c}5.6910^{-19} \\
5.5610^{-16} \\
1.1610^{-9} \\
1.1610^{-9}\end{array}$ & $\begin{array}{l}2.9910^{-37} \\
1.6810^{-32} \\
8.5410^{-10} \\
8.5410^{-10}\end{array}$ & $\begin{array}{c}9.5910^{-27} \\
4.9210^{-24} \\
1.4310^{-9} \\
1.4310^{-9}\end{array}$ & $\begin{array}{l}3.2310^{-21} \\
3.3810^{-18} \\
4.3510^{-12} \\
4.3510^{-12}\end{array}$ \\
\hline${ }^{14} \mathrm{~N}$ & $\begin{array}{l}\text { at } R G \text { top } \\
\text { at } t_{0} \\
\text { at } A G B \text { tip } \\
\text { net yield }\end{array}$ & $\begin{array}{l}3.4110^{-6} \\
2.3510^{-4} \\
5.2410^{-3} \\
3.0110^{-3}\end{array}$ & $\begin{array}{l}2.2210^{-6} \\
6.5310^{-4} \\
8.5210^{-3} \\
5.0310^{-3}\end{array}$ & $\begin{array}{l}3.9210^{-6} \\
9.2610^{-4} \\
1.8410^{-2} \\
1.3810^{-2}\end{array}$ & $\begin{array}{l}2.9910^{-6} \\
9.0110^{-4} \\
2.8910^{-2} \\
2.3510^{-2}\end{array}$ & $\begin{array}{l}3.2510^{-7} \\
1.5310^{-4} \\
1.5810^{-3} \\
9.7510^{-4}\end{array}$ & $\begin{array}{l}4.4710^{-8} \\
3.6010^{-4} \\
2.0710^{-3} \\
1.2610^{-3}\end{array}$ & $\begin{array}{l}1.6910^{-7} \\
4.6610^{-4} \\
1.0410^{-2} \\
9.3310^{-3}\end{array}$ \\
\hline${ }^{15} \mathrm{~N}$ & $\begin{array}{l}\text { at } R G \text { top } \\
\text { at } t_{0} \\
\text { at } A G B \text { tip } \\
\text { net yield }\end{array}$ & $\begin{array}{c}8.8910^{-9} \\
1.9210^{-7} \\
4.1310^{-6} \\
-4.6510^{-6}\end{array}$ & $\begin{array}{c}5.8310^{-9} \\
4.9310^{-7} \\
6.3210^{-6} \\
-7.4710^{-6}\end{array}$ & $\begin{array}{c}1.2810^{-8} \\
6.3310^{-7} \\
1.9710^{-6} \\
-1.6210^{-5}\end{array}$ & $\begin{array}{c}8.0210^{-9} \\
5.8910^{-7} \\
2.6610^{-6} \\
-1.8710^{-5}\end{array}$ & $\begin{array}{c}2.4010^{-10} \\
1.0110^{-7} \\
1.0210^{-6} \\
-1.3510^{-6}\end{array}$ & $\begin{array}{c}4.3610^{-11} \\
2.5610^{-7} \\
9.1310^{-7} \\
-2.2610^{-6}\end{array}$ & $\begin{array}{c}2.0210^{-10} \\
3.7010^{-7} \\
5.1310^{-6} \\
9.5310^{-7}\end{array}$ \\
\hline
\end{tabular}

\section{Conclusions and prospects}

We tried to confirm and improve our present knowledge of the structural and nucleosynthetic evolution of intermediate-mass AGB stars. To do that, we have first presented in detail (i) the physics and numerical aspects of our stellar evolution code, (ii) results concerning evolution phases prior to the AGB one and (iii) comparisons with other works. These informations are essential to better understand and appraise the large set of predictions we discuss about the thermally pulsing AGB stage. We also compare our predictions with various observations (at different evolutionary stages) in order to clearly identify the processes that should be included in future models.

Let us just mention global key features stemming from our intermediate-mass evolution models.

- Concerning the structure (intensities, temperatures) of the thermal pulses as well as the thermal properties at the base of the convective envelope, the most 
Table 9. Same as Table 8 for elements from $\mathrm{O}$ to $\mathrm{Na}$

\begin{tabular}{|c|c|c|c|c|c|c|c|c|}
\hline & & \multicolumn{4}{|c|}{$Z=0.02$} & \multicolumn{3}{|c|}{$Z=0.005$} \\
\hline & & $3 M_{\odot}$ & $4 M_{\odot}$ & $5 M_{\odot}$ & $6 M_{\odot}$ & $3 M_{\odot}$ & $4 M_{\odot}$ & $5 M_{\odot}$ \\
\hline${ }^{16} \mathrm{O}$ & $\begin{array}{l}\text { at } R G \text { top } \\
\text { at } t_{0} \\
\text { at } A G B \text { tip } \\
\text { net yield }\end{array}$ & $\begin{array}{c}2.2310^{-5} \\
8.3310^{-4} \\
1.9210^{-2} \\
-1.3610^{-4}\end{array}$ & $\begin{array}{c}1.4710^{-5} \\
2.2010^{-3} \\
2.8610^{-2} \\
-1.6610^{-3}\end{array}$ & $\begin{array}{c}3.0010^{-5} \\
2.8410^{-3} \\
3.6110^{-2} \\
-3.7210^{-3}\end{array}$ & $\begin{array}{c}2.0010^{-5} \\
2.6710^{-3} \\
3.9610^{-2} \\
-7.3310^{-3}\end{array}$ & $\begin{array}{l}1.1010^{-6} \\
4.6810^{-4} \\
5.3410^{-3} \\
1.2710^{-4}\end{array}$ & $\begin{array}{c}1.9210^{-7} \\
1.2110^{-3} \\
6.7410^{-3} \\
-2.2910^{-4}\end{array}$ & $\begin{array}{c}8.2210^{-7} \\
1.6910^{-3} \\
4.1710^{-3} \\
-5.0110^{-3}\end{array}$ \\
\hline${ }^{17} \mathrm{O}$ & $\begin{array}{l}\text { at } R G \text { top } \\
\text { at } t_{0} \\
\text { at } A G B \text { tip } \\
\text { net yield }\end{array}$ & $\begin{array}{l}2.1810^{-8} \\
2.6210^{-6} \\
5.8710^{-5} \\
5.0910^{-5}\end{array}$ & $\begin{array}{l}1.1510^{-8} \\
5.5610^{-6} \\
7.2610^{-5} \\
6.0310^{-5}\end{array}$ & $\begin{array}{l}1.5110^{-8} \\
5.4510^{-6} \\
7.1410^{-5} \\
5.5210^{-5}\end{array}$ & $\begin{array}{l}1.2610^{-8} \\
4.5110^{-6} \\
1.1710^{-4} \\
9.8010^{-5}\end{array}$ & $\begin{array}{l}4.1910^{-9} \\
1.8710^{-6} \\
1.9410^{-5} \\
1.7310^{-5}\end{array}$ & $\begin{array}{c}2.0910^{-10} \\
3.6310^{-6} \\
2.0310^{-5} \\
1.7510^{-5}\end{array}$ & $\begin{array}{l}4.3810^{-10} \\
3.1710^{-6} \\
1.0510^{-5} \\
6.7810^{-6}\end{array}$ \\
\hline${ }^{18} \mathrm{O}$ & $\begin{array}{l}\text { at } R G \text { top } \\
\text { at } t_{0} \\
\text { at } A G B \text { tip } \\
\text { net yield }\end{array}$ & $\begin{array}{c}4.7410^{-8} \\
1.4210^{-6} \\
3.1010^{-5} \\
-1.2610^{-5}\end{array}$ & $\begin{array}{c}3.1310^{-8} \\
3.7610^{-6} \\
4.8410^{-5} \\
-2.0110^{-5}\end{array}$ & $\begin{array}{c}6.6110^{-8} \\
4.8710^{-6} \\
2.0910^{-5} \\
-6.9110^{-5}\end{array}$ & $\begin{array}{c}4.2910^{-8} \\
4.5710^{-6} \\
5.3210^{-6} \\
-1.0110^{-4}\end{array}$ & $\begin{array}{c}1.7910^{-9} \\
7.5510^{-7} \\
7.6610^{-6} \\
-4.1110^{-6}\end{array}$ & $\begin{array}{c}3.3610^{-10} \\
1.9710^{-6} \\
1.0610^{-5} \\
-5.1610^{-6}\end{array}$ & $\begin{array}{c}1.5910^{-9} \\
2.8610^{-6} \\
3.8110^{-6} \\
-1.6910^{-5}\end{array}$ \\
\hline${ }^{19} \mathrm{~F}$ & $\begin{array}{l}\text { at } R G \text { top } \\
\text { at } t_{0} \\
\text { at } A G B \text { tip } \\
\text { net yield }\end{array}$ & $\begin{array}{l}9.5410^{-10} \\
3.7010^{-8} \\
1.8710^{-6} \\
1.0610^{-6}\end{array}$ & $\begin{array}{c}6.2310^{-10} \\
9.5410^{-8} \\
1.6810^{-6} \\
3.9610^{-7}\end{array}$ & $\begin{array}{l}1.2710^{-9} \\
1.2210^{-7} \\
2.0710^{-6} \\
3.8410^{-7}\end{array}$ & $\begin{array}{c}8.4610^{-10} \\
1.1310^{-7} \\
1.7510^{-6} \\
-2.3310^{-7}\end{array}$ & $\begin{array}{l}4.8810^{-11} \\
2.0710^{-8} \\
4.3110^{-7} \\
2.1110^{-7}\end{array}$ & $\begin{array}{l}8.3810^{-12} \\
5.2710^{-8} \\
3.4110^{-7} \\
4.6510^{-8}\end{array}$ & $\begin{array}{c}3.5510^{-11} \\
7.3410^{-8} \\
1.6210^{-7} \\
-2.2610^{-7}\end{array}$ \\
\hline${ }^{20} \mathrm{Ne}$ & $\begin{array}{l}\text { at } R G \text { top } \\
\text { at } t_{0} \\
\text { at } A G B \text { tip } \\
\text { net yield }\end{array}$ & $\begin{array}{c}3.7910^{-6} \\
1.4610^{-4} \\
3.2510^{-3} \\
-1.8710^{-6}\end{array}$ & $\begin{array}{c}2.4910^{-6} \\
3.8910^{-4} \\
5.0510^{-3} \\
-6.5110^{-5}\end{array}$ & $\begin{array}{c}5.0710^{-6} \\
5.1210^{-4} \\
6.6910^{-3} \\
-3.0310^{-5}\end{array}$ & $\begin{array}{c}3.3910^{-6} \\
4.8510^{-4} \\
7.9010^{-3} \\
-2.9810^{-5}\end{array}$ & $\begin{array}{c}1.9210^{-7} \\
8.3410^{-5} \\
8.6410^{-4} \\
-1.5110^{-5}\end{array}$ & $\begin{array}{c}3.2410^{-8} \\
2.1110^{-4} \\
1.1710^{-3} \\
-4.5310^{-6}\end{array}$ & $\begin{array}{l}1.3910^{-7} \\
2.9310^{-4} \\
1.5810^{-3} \\
2.5910^{-5}\end{array}$ \\
\hline${ }^{21} \mathrm{Ne}$ & $\begin{array}{l}\text { at } R G \text { top } \\
\text { at } t_{0} \\
\text { at } A G B \text { tip } \\
\text { net yield }\end{array}$ & $\begin{array}{l}9.8110^{-9} \\
3.8310^{-7} \\
1.0110^{-5} \\
1.7910^{-6}\end{array}$ & $\begin{array}{l}6.4510^{-9} \\
1.0310^{-6} \\
1.4210^{-5} \\
1.2110^{-6}\end{array}$ & $\begin{array}{c}1.3110^{-8} \\
1.3510^{-6} \\
9.2910^{-6} \\
-7.8510^{-6}\end{array}$ & $\begin{array}{l}8.7610^{-9} \\
1.2710^{-6} \\
2.7110^{-6} \\
-1.7510^{-5}\end{array}$ & $\begin{array}{l}5.3110^{-10} \\
2.3710^{-7} \\
2.8310^{-6} \\
5.9210^{-7}\end{array}$ & $\begin{array}{l}8.7510^{-11} \\
5.8610^{-7} \\
3.3610^{-6} \\
3.6010^{-7}\end{array}$ & $\begin{array}{c}3.7110^{-10} \\
7.8510^{-7} \\
1.1510^{-6} \\
-2.8010^{-6}\end{array}$ \\
\hline${ }^{22} \mathrm{Ne}$ & $\begin{array}{l}\text { at } R G \text { top } \\
\text { at } t_{0} \\
\text { at } A G B \text { tip } \\
\text { net yield }\end{array}$ & $\begin{array}{l}2.9910^{-7} \\
1.0610^{-5} \\
9.4510^{-4} \\
6.8310^{-4}\end{array}$ & $\begin{array}{l}1.9710^{-7} \\
2.8110^{-5} \\
6.8110^{-4} \\
2.6910^{-4}\end{array}$ & $\begin{array}{l}4.0610^{-7} \\
3.6610^{-5} \\
7.7510^{-4} \\
2.3510^{-4}\end{array}$ & $\begin{array}{l}2.6910^{-7} \\
3.4510^{-5} \\
8.9410^{-4} \\
2.5610^{-4}\end{array}$ & $\begin{array}{l}1.3810^{-8} \\
5.8510^{-6} \\
2.4010^{-4} \\
1.6910^{-4}\end{array}$ & $\begin{array}{l}2.5410^{-9} \\
1.5410^{-5} \\
1.3910^{-4} \\
4.4410^{-5}\end{array}$ & $\begin{array}{l}1.1110^{-8} \\
2.2110^{-5} \\
1.4410^{-4} \\
1.9810^{-5}\end{array}$ \\
\hline${ }^{23} \mathrm{Na}$ & $\begin{array}{l}\text { at } R G \text { top } \\
\text { at } t_{0} \\
\text { at } A G B \text { tip } \\
\text { net yield }\end{array}$ & $\begin{array}{l}8.4010^{-8} \\
4.1110^{-6} \\
9.4710^{-5} \\
2.7610^{-5}\end{array}$ & $\begin{array}{l}5.4810^{-8} \\
1.1310^{-5} \\
1.4910^{-4} \\
4.3010^{-5}\end{array}$ & $\begin{array}{l}1.0710^{-7} \\
1.5510^{-5} \\
2.1610^{-4} \\
7.7210^{-5}\end{array}$ & $\begin{array}{l}7.4110^{-8} \\
1.4910^{-5} \\
2.5110^{-4} \\
8.8010^{-5}\end{array}$ & $\begin{array}{l}5.6610^{-9} \\
2.6010^{-6} \\
2.8710^{-5} \\
1.0610^{-5}\end{array}$ & $\begin{array}{l}7.3310^{-10} \\
6.0810^{-6} \\
3.5010^{-5} \\
1.0710^{-5}\end{array}$ & $\begin{array}{c}2.9210^{-9} \\
7.7610^{-6} \\
2.8010^{-5} \\
-3.9810^{-6}\end{array}$ \\
\hline
\end{tabular}

decisive quantities are the total mass and core mass. Both change with time in a way that is governed by the increasing mass loss rate. This translates into a great sensitivity of the various nucleosynthesis processes to the adopted mass loss rate. Consequently, the evolution of the thermal pulse characteristics crucially depend on this last quantity, unfortunately still rather badly known. In addition, the lower the mass loss rates, the longer the TP-AGB phase, the more third dredge-up events have time to occur and the greater are the surface abundance changes due to $\mathrm{HBB}$. This is clearly demonstrated by the high sensitiveness of the predicted surface evolution of almost all the isotopic ratios to the mass loss rate dependence on time.

- On the other hand, at a given total stellar mass, core masses are greater for lower metallicities, as a result of the central burning phases. As a consequence, for the reason mentioned at the former point, TP-AGB stars with $Z=0.005$ mostly behave like $Z=0.02$ ones with a total mass greater by $\sim 1 M_{\odot}$. However, due to important differences in initial compositions that also influence the nuclear reactions, this is not completely true as far as isotopic ratios are concerned. At the end of the TP-AGB phase indeed, some yields are quite different for both $Z$ we have studied.

- The neutron captures on intermediate-mass and heavy nuclides occur in two distinct sites inside TP-AGB stars, namely (i) at the base of the inter-shell zone during the inter-pulse phase and, as already known, (ii) inside the convective tongues associated with thermal pulses. Along the asymptotic TP-AGB, the amount of produced neutrons is rather independent of the initial 
Table 10. Same as Table 8 for elements from $\mathrm{Mg}$ to $\mathrm{Si}$

\begin{tabular}{|c|c|c|c|c|c|c|c|c|}
\hline & & \multicolumn{4}{|c|}{$Z=0.02$} & \multicolumn{3}{|c|}{$Z=0.005$} \\
\hline & & $3 M_{\odot}$ & $4 M_{\odot}$ & $5 M_{\odot}$ & $6 M_{\odot}$ & $3 M_{\odot}$ & $4 M_{\odot}$ & $5 M_{\odot}$ \\
\hline${ }^{24} \mathrm{Mg}$ & $\begin{array}{l}\text { at } R G \text { top } \\
\text { at } t_{0} \\
\text { at } A G B \text { tip } \\
\text { net yield }\end{array}$ & $\begin{array}{c}1.2010^{-6} \\
4.6210^{-5} \\
1.0310^{-3} \\
-1.3510^{-6}\end{array}$ & $\begin{array}{c}7.9010^{-7} \\
1.2310^{-4} \\
1.6010^{-3} \\
-2.5510^{-5}\end{array}$ & $\begin{array}{c}1.6110^{-6} \\
1.6310^{-4} \\
2.1210^{-3} \\
-2.0910^{-5}\end{array}$ & $\begin{array}{c}1.0810^{-6} \\
1.5410^{-4} \\
2.3110^{-3} \\
-2.0910^{-4}\end{array}$ & $\begin{array}{c}6.0910^{-8} \\
2.6410^{-5} \\
2.7410^{-4} \\
-6.0510^{-6}\end{array}$ & $\begin{array}{c}1.0310^{-8} \\
6.6810^{-5} \\
3.7010^{-4} \\
-4.7310^{-6}\end{array}$ & $\begin{array}{c}4.4010^{-8} \\
9.2910^{-5} \\
2.2810^{-4} \\
-2.6510^{-4}\end{array}$ \\
\hline${ }^{25} \mathrm{Mg}$ & $\begin{array}{l}\text { at } R G \text { top } \\
\text { at } t_{0} \\
\text { at } A G B \text { tip } \\
\text { net yield }\end{array}$ & $\begin{array}{l}1.6010^{-7} \\
6.1210^{-6} \\
1.4210^{-4} \\
6.1710^{-6}\end{array}$ & $\begin{array}{l}1.0510^{-7} \\
1.6310^{-5} \\
2.2210^{-4} \\
7.7210^{-6}\end{array}$ & $\begin{array}{l}2.1410^{-7} \\
2.1110^{-5} \\
2.8410^{-4} \\
3.1910^{-6}\end{array}$ & $\begin{array}{l}1.4210^{-7} \\
1.9910^{-5} \\
5.1710^{-4} \\
1.8510^{-4}\end{array}$ & $\begin{array}{l}8.2610^{-9} \\
3.5710^{-6} \\
3.8810^{-5} \\
2.0010^{-6}\end{array}$ & $\begin{array}{l}1.4010^{-9} \\
9.0410^{-6} \\
5.1310^{-5} \\
2.0610^{-6}\end{array}$ & $\begin{array}{l}5.9610^{-9} \\
1.2410^{-5} \\
1.7310^{-4} \\
1.0810^{-4}\end{array}$ \\
\hline${ }^{26} \mathrm{Mg}$ & $\begin{array}{l}\text { at } R G \text { top } \\
\text { at } t_{0} \\
\text { at } A G B \text { tip } \\
\text { net yield }\end{array}$ & $\begin{array}{l}1.8210^{-7} \\
7.0010^{-6} \\
1.6810^{-4} \\
1.2310^{-5}\end{array}$ & $\begin{array}{l}1.2010^{-7} \\
1.8710^{-5} \\
2.5710^{-4} \\
1.2210^{-5}\end{array}$ & $\begin{array}{l}2.4410^{-7} \\
2.5110^{-5} \\
3.4810^{-4} \\
2.5210^{-5}\end{array}$ & $\begin{array}{l}1.6310^{-7} \\
2.3910^{-5} \\
4.0610^{-4} \\
2.5710^{-5}\end{array}$ & $\begin{array}{l}9.3110^{-9} \\
4.0510^{-6} \\
4.5010^{-5} \\
2.8410^{-6}\end{array}$ & $\begin{array}{l}1.5610^{-9} \\
1.0310^{-5} \\
5.9810^{-5} \\
3.3910^{-6}\end{array}$ & $\begin{array}{c}6.6910^{-9} \\
1.4410^{-5} \\
5.4310^{-5} \\
-1.9910^{-5}\end{array}$ \\
\hline${ }^{26} \mathrm{Al}^{\mathrm{g}}$ & $\begin{array}{l}\text { at } R G \text { top } \\
\text { at } t_{0} \\
\text { at } A G B \text { tip } \\
\text { net yield }\end{array}$ & $\begin{array}{c}1.2010^{-16} \\
6.1510^{-16} \\
2.0310^{-7} \\
2.0310^{-7}\end{array}$ & $\begin{array}{c}2.8310^{-15} \\
1.3910^{-10} \\
8.8710^{-8} \\
8.8710^{-8}\end{array}$ & $\begin{array}{l}1.0110^{-15} \\
5.6310^{-9} \\
4.0310^{-7} \\
4.0310^{-7}\end{array}$ & $\begin{array}{l}1.9310^{-14} \\
2.4410^{-8} \\
3.6710^{-6} \\
3.6710^{-6}\end{array}$ & $\begin{array}{c}1.2910^{-17} \\
3.1010^{-14} \\
4.3410^{-8} \\
4.3410^{-8}\end{array}$ & $\begin{array}{c}4.2910^{-21} \\
6.1210^{-10} \\
9.0210^{-9} \\
9.0210^{-9}\end{array}$ & $\begin{array}{l}1.1110^{-21} \\
2.7310^{-9} \\
7.6910^{-6} \\
7.6910^{-6}\end{array}$ \\
\hline${ }^{27} \mathrm{Al}$ & $\begin{array}{l}\text { at } R G \text { top } \\
\text { at } t_{0} \\
\text { at } A G B \text { tip } \\
\text { net yield }\end{array}$ & $\begin{array}{l}1.3610^{-7} \\
5.2110^{-6} \\
1.1810^{-4} \\
1.4910^{-6}\end{array}$ & $\begin{array}{c}8.9110^{-8} \\
1.3910^{-5} \\
1.8310^{-4} \\
-5.2610^{-8}\end{array}$ & $\begin{array}{l}1.8210^{-7} \\
1.8410^{-5} \\
2.4510^{-4} \\
3.7810^{-6}\end{array}$ & $\begin{array}{l}1.2210^{-7} \\
1.7410^{-5} \\
2.9410^{-4} \\
1.0010^{-5}\end{array}$ & $\begin{array}{l}6.8910^{-9} \\
2.9910^{-6} \\
3.2710^{-5} \\
1.2410^{-6}\end{array}$ & $\begin{array}{l}1.1610^{-9} \\
7.5710^{-6} \\
4.3810^{-5} \\
1.6010^{-6}\end{array}$ & $\begin{array}{l}4.9710^{-9} \\
1.0510^{-5} \\
7.4210^{-5} \\
1.8710^{-5}\end{array}$ \\
\hline${ }^{28} \mathrm{Si}$ & $\begin{array}{l}\text { at } R G \text { top } \\
\text { at } t_{0} \\
\text { at } A G B \text { tip } \\
\text { net yield }\end{array}$ & $\begin{array}{c}1.5310^{-6} \\
5.8710^{-5} \\
1.3110^{-3} \\
-1.4710^{-6}\end{array}$ & $\begin{array}{c}1.0010^{-6} \\
1.5710^{-4} \\
2.0410^{-3} \\
-2.7610^{-5}\end{array}$ & $\begin{array}{c}2.0510^{-6} \\
2.0710^{-4} \\
2.7010^{-3} \\
-1.3510^{-5}\end{array}$ & $\begin{array}{c}1.3710^{-6} \\
1.9610^{-4} \\
3.1810^{-3} \\
-1.8410^{-5}\end{array}$ & $\begin{array}{c}7.7610^{-8} \\
3.3610^{-5} \\
3.4910^{-4} \\
-5.9510^{-6}\end{array}$ & $\begin{array}{c}1.3110^{-8} \\
8.5210^{-5} \\
4.7310^{-4} \\
-2.2010^{-6}\end{array}$ & $\begin{array}{c}5.6010^{-8} \\
1.1810^{-4} \\
6.0710^{-4} \\
-1.8510^{-5}\end{array}$ \\
\hline
\end{tabular}

total mass and metallicity (at least for intermediatemass objects).

- Concerning chemical evolution of galaxies, intermediate-mass stars, at the end of their existence, appear to be rather significant producers of ${ }^{3} \mathrm{He},{ }^{4} \mathrm{He}$, ${ }^{7} \mathrm{Li}$ (most massive and/or higher $Z$ stars), ${ }^{13} \mathrm{C},{ }^{14} \mathrm{~N}$, ${ }^{17} \mathrm{O},{ }^{19} \mathrm{~F},{ }^{22} \mathrm{Ne},{ }^{23} \mathrm{Na},{ }^{26} \mathrm{Mg}$ and ${ }^{27} \mathrm{Al}$. A found trend, compared to observations, however clearly supports the idea that most of the ${ }^{19} \mathrm{~F}$ present in the interstellar medium has to come from low-mass AGB stars (that point has to be confirmed by forthcoming evolutionary models).

Intermediate-mass AGB stars also substantially produce radio-nuclides, namely ${ }^{14} \mathrm{C}$ and ${ }^{26} \mathrm{Al}$. The former one mostly comes from lower mass stars while it is the contrary for the last one.

These stars partially deplete the interstellar medium content in ${ }^{7} \mathrm{Li}$ (less massive stars), ${ }^{15} \mathrm{~N},{ }^{16} \mathrm{O},{ }^{18} \mathrm{O},{ }^{20} \mathrm{Ne}$, ${ }^{24} \mathrm{Mg}$ and ${ }^{28} \mathrm{Si}$.

We finish by recalling the major problems that, among all the present intermediate-mass AGB models, remain to be solved.
- We have not yet identified the ${ }^{13} \mathrm{C}$ additional source needed to quantitatively explain the s-process. This process is however required in order to account for many observations unquestionably indicating that AGB stars are responsible for the main component of the solar system heavy element distribution. Some suggestions have been made. Radiative diffusion operating during the TP-AGB phase of low-mass stars could substantially help. Although already invoked, such a process has never been yet included in AGB star evolution models.

- The rather high level of ${ }^{7} \mathrm{Li}$ production detected at the surface of some evolved but relatively faint AGB stars of our galaxy still remains unaccountable. Thus, the Cameron-Fowler mechanism does not explain all the super-lithium-rich AGB stars. As these galactic objects have relatively low initial masses, the explanation could also come from the radiative slow-transport processes above mentioned.

- Last but not least, some C stars are observed, especially in the galactic bulge, with low luminosities at which the models fail at predicting the occurrence of the third dredge-up. This is probably due to our 
bad knowledge (and treatment) of finely tuned convection motions in boundaries of stellar regions having strong chemical composition gradients. Clearly, some kind of extra-mixing (deeper inside nuclearly processed regions) has to be invoked. However, no self-consistent mechanism has been settled and tested through complete evolution models up to now.

Other confrontations with observations require the modeling of low-mass AGB stars. A clear distinction between low- and intermediate-mass stars is justified. Indeed, numerous observations indicate that "nonstandard" particle transport processes are acting inside low-mass stars at different phases of their evolution, that substantially modify the chemical structure compared to what is obtained in classical models. During the various dredge-up episodes, matter up-heaved to the surface has consequently a different composition. This is not the case in intermediate-mass stars. In conclusion, low-mass AGB stellar models, maintaining to make detailed predictions concerning the evolution of surface isotopic ratios, have to include such slow-particle transport processes. They are currently being calculated and will be presented in a next-coming paper.

Acknowledgements. We are first indebted to Maurizio Busso, the referee, for its very careful reading of our manuscript that helped us to significantly improve and clarify it. We also thank him and Roberto Gallino for the very instructive discussions we already had together about these very complex AGB stars. Let us finally thank Lionel Siess for his always valuable contributions to improve the stellar evolution code and related utilities. Part of the computations presented in this paper (roughly representing 7 months of CPU time) were performed at the "Center de Calcul Intensif de l'Observatoire de Grenoble". Most of them have been realized at "IMAG" on a IBM SP1 computer financed by the MESR, CNRS and Région Rhône-Alpes. This work was supported by grants from the GDR "Structure Interne des Etoiles et des Planètes Géantes" (CNRS).

\section{References}

Aaronson M., Mould J., 1985, ApJ 288, 551

Abia C., Boffin H.M.J., Isern J., Rebolo R., 1991, A\&A 245, L1

Abia C., Boffin H.M.J., Isern J., Rebolo R., 1993, A\&A 272, 455

Alexander D.R., Ferguson J.W., 1994, ApJ 437, 879

Allen B.J., Machlin R.L., Gibbons J.H., 1971, Adv. Nucl. Phys. 4, 205

Alongi M., Bertelli G., Bressan A., Chiosi C., 1991, in Formation and Evolution of Star Clusters, Kanneth J. (ed.) ASP Conf. Ser. 13, 223
Anders E., Grevesse N., 1989, Geochim. Cosmochim. Acta 53, 197

Bao Z.Y., Käppeler F., 1987, Atomic Data Nucl. Data Tab. 36, 411

Bazan G., Lattanzio J.C., 1993, ApJ 409, 762

Beer H., Wiescher M., Käppeler F., Gorres J., Koehler P.E., 1991 (preprint)

Bell R.A., Eriksson K., Gustafsson B., Nordlund A., 1976, A\&A 23, 37

Bergeron P., Saffer R., Liebert J., 1991, in Confrontation between Stellar Pulsation and Evolution, Cacciari C. and Clementini G. (eds.) p. 513

Blanco B.M., Blanco V.M., McCarthy M.F., 1978, Nat 271, 638

Blanco V.M., McCarthy M.F., Blanco B.M., 1980, ApJ 242, 938

Blöcker T., 1995, A\&A 297, 727

Blöcker T., Schönberner D., 1991, A\&A 244, L43

Boothroyd A.I., Sackmann I.-J., 1988a, ApJ 328, 641

Boothroyd A.I., Sackmann I.-J., 1988b, ApJ 328, 653

Boothroyd A.I., Sackmann I.-J., 1988c, ApJ 328, 671

Boothroyd A.I., Sackmann I.-J., Ahern S.C., 1993, ApJ 416, 762

Boothroyd A.I., Sackmann I.-J., Wasserburg G.J., 1994, ApJ 430, L77

Boothroyd A.I., Sackmann I.-J., Wasserburg G.J., 1995, ApJ 442, L21

Brehm K., Becker H.W., Rolfs C., Käppeler F., Ratynski W., 1988, Phys. A. Atomic Nuclei 330, 167

Bressan A., Fagotto F., Bertelli G., Chiosi C., 1993, A\&AS 100, 647

Bryan G.L., Volk K., Kwok S., 1990, ApJ 365, 301

Busso M., Lambert D.L., Beglio L., Gallino R., Raiteri C.M., Smith V.V., 1995, ApJ 446, 775

Cameron A.G.W., Fowler W.A., 1971, ApJ 164, 111

Caughlan G.R., Fowler W.A., 1988, Atomic Data Nucl. Data Tab. 40,283

Caughlan G.R., Fowler W.A., Harris M.J., Zimmerman B.A., 1985, Atomic Data Nucl. Data Tab. 32, 197

Champagne A.E., Cella C.H., Konzes R.T., Loury R.M., Magnus P.V., Smith M.S., Mao Z.Q., 1988, Nucl. Phys. A 487,433

Charbonnel C., 1994, A\&A 282, 811

Charbonnel C., 1995, ApJ 453, L41

Chieffi A., Straniero O., Salaris M., 1995, ApJ 445, L39

Claussen M.J., Kleinmann S.G., Joyce R.R., Jura M., 1987, ApJS 65, 385

Cohen J.G., Frogel J.A., Persson S.E., Elias J.H., 1981, ApJ 249,481

De Oliveira F., 1995, Ph. D. Thesis, Université de Paris XI, France

El Eid M.F., 1994, A\&A 285, 915

Feast M.W., 1974, in Stellar Instability and Evolution, Ledoux P., Noels A. and Rodgers A.W. (eds.). Dordrecht, Reidel, p. 93

Forestini M., Guélin M., Cernicharo J., 1996, A\&A (in press)

Forestini M., Paulus G., Arnould M., 1991, A\&A 252, 597

Forestini M., Goriely S., Jorissen A., Arnould M., 1992, A\&A 261, 157

Fowler W.A., Caughlan G.R., Zimmerman B.A., 1967, ARA\&A 5, 525 
Frost J.C., Lattanzio J., 1996, ApJ (in press)

Fujimoto M.Y., Sugimoto D., 1979, PASJ 31, 1

Funck C., Langanke K., 1989, ApJ 344, 46

Gilroy K.K., 1989, ApJ 347, 835

Graboske H.C., DeWitt H.E., Grossman A.S., Cooper M.S., 1973, ApJ 181, 457

Grevesse N., 1991, in Evolution of Stars: The Photospheric Abundance Connection, Michaud G. and Tutukov A. (eds.). Dordrecht, Kluwer, p. 63

Griffin I.P., 1990, MNRAS 247, 591

Groenewegen M.A.T., de Jong T., 1993, A\&A 267, 410

Guélin M., Lucas R., Cernicharo J., 1993, A\&A 280, L19

Guélin M., Forestini M., Valiron P., Ziurys L.M., Anderson M.A., Cernicharo J., Kahane C., 1995, A\&A 297, 183

Hammer J.W., 1991 (preprint)

Harris M.J., Lambert D.L., 1984, ApJ 281, 739

Harris M.J., Lambert D.L., 1987, ApJ 318, 868

Harris M.J., Lambert D.L., Smith V.V., 1985, ApJ 299, 375

Harris M.J., Lambert D.L., Smith V.V., 1988, ApJ 321, 761

Harris M.J., Lambert D.L., Hinkle K.H., Gustafsson B., Eriksson K., 1987, ApJ 316, 294

Hubbard W.B., Lampe M., 1969, ApJS 18, 297

Huebner W.F., Mertz A.L., Magee N.H., Argo M.F., 1977, in Astrophys. Opacity Library, UC-346

Huss G.R., Fahey A.J., Gallino R., Wasserburg G.J., 1994, ApJ 430, L81

Iben I., 1964, ApJ 140, 1631

Iben I., 1975, ApJ 196, 525

Iben I., 1976, ApJ 208, 165

Iben I., 1981, ApJ 246, 278

Iben I., 1983, ApJ 275, L65

Illiadis C., 1990, Nucl. Phys. A 512, 509

Itoh N., Mutoh H., Hikita A., Kohyama Y., 1992, ApJ 395, 622

Jorissen A., Arnould M., 1989, A\&A 221, 161

Jorissen A., Smith V.V., Lambert D.L., 1992, A\&A 261, 164

Kahane C., Gómez-Gonzáles J., Cernicharo J., Guélin M., 1988, A\&A 190, 167

Kahane C., Cernicharo J., Gómez-Gonzáles J., Guélin M., 1992, A\&A 256, 235

Käppeler F., Gallino R., Busso M., Picchio G., Raiteri C.M., 1990, ApJ 354, 630

Käppeler F., et al., 1994, ApJ 437, 396

Keady J.J., Hall D.N.B., Ridgway S.T., 1988, ApJ 326, 832

Kious M., 1990, Ph.D. Thesis, Université de Paris XI, France

Kippenhahn R., Weigert A., 1991, Stellar Structure and Evolution. Springer-Verlag, p. 23

Kippenhahn R., Weigert A., Hofmeister E., 1968, Meth. Comp. Phys. 7, 129

Knapp G.R., et al., 1989, ApJ 336, 822

Koester D., 1976, A\&A 52, 415

Kohyama Y., Itoh N., Obama A., Mutoh H., 1993, ApJ 415, 267

Lambert D.L., Gustafsson B., Eriksson K., Hinkle K.H., 1986, ApJS 62, 373

Lambert D.L., Smith V.V., Busso M., Gallino R., Straniero O., 1995, ApJ 450, 302

Landré V., Prantzos N., Aguer P., Bogaert G., Lefèbvre A., Thibaud J.-P., 1990, A\&A 240, 85

Lattanzio J., 1986, ApJ 311, 708

Lattanzio J., 1987, ApJ 313, L15
Lattanzio J., 1989, in Evolution of Peculiar Red Giants, Johnson H.R. and Zuckerman B. (eds.). Cambridge University Press, Cambridge, p. 161

Malaney R.A., Boothroyd A.I., 1987, ApJ 320, 866

Malaney R.A., Savage M.J., Boothroyd A.I., 1988, ApJ 324, 948

Merrill P.W., 1952, ApJ 116, 21

Mould J.R., Aaronson M., 1982, ApJ 263, 629

Mould J.R., Aaronson M., 1986, ApJ 303, 10

Munakata H., Kohyama Y., Itoh N., 1985, ApJ 296, 197

Nagai Y., Igashira M., Takeda K., et al., 1991, ApJ 372, 683

Nagai Y., et al., 1995, in Nuclei in the Cosmos III, Busso M., Gallino R. and Raiteri C.M. (eds.) AIP, New York, p. 201

Oke J.B., Weideman V., Koester D., 1984, ApJ 281, 276

Ott U., 1991, Protostars and Planets III. University of Arizona Press, Tuscon, p. 144

Paczyński B., 1969, Acta Astron. 19, 1

Paczyński B., 1975, ApJ 202, 558

Plez B., 1992, A\&AS 94, 527

Plez B., Smith V.V., Lambert D.L., 1993, ApJ 418, 812

Press W.H., Flannery B.P., Teukolsky S.A., Vetterling W.T., 1986, Numerical Recipes. Cambridge University Press, p. 588

Raman S., Igashira M., Dozono Y., Kitazawa H., Mizimoto M., Lynn J.E., 1990, Phys. Rev. C 41, 458

Reid N., Mould J., 1984, ApJ 284, 98

Reimers D., 1975, Mem. Soc. Roy. Sci. Liège, 6th Ser. 8, 369

Richard O., Vauclair S., Charbonnel C., Dziembowski W.A., 1996, A\&A (in press)

Rogers F.J., Iglesias C.A., 1992, ApJS 79, 507

Sackmann I.-J., 1980, ApJ 235, 554

Sackmann I.-J., Boothroyd A.I., 1991a, ApJ 366, 529

Sackmann I.-J., Boothroyd A.I., 1991b, in IAU Symp. 145, Michaud G. and Tutukov A. (eds.). Dordrecht, Reidel, p. 275

Sackmann I.-J., Boothroyd A.I., 1992, ApJ 392, L71

Sackmann I.-J., Smith R.L., Despain K.H., 1974, ApJ 187, 555

Scalo J.M., Despain K.H., Ulrich R.K., 1975, ApJ 196, 805

Schaller G., Schaerer D., Meynet G., Maeder A., 1992, A\&AS 96, 269

Schwarzschild M., Härm R., 1965, ApJ 142, 855

Smith V.V., Lambert D.L., 1986, ApJ 311, 843

Smith V.V., Lambert D.L., 1989, ApJ 345, L75

Smith V.V., Lambert D.L., 1990a, ApJS 72, 387

Smith V.V., Lambert D.L., 1990b, ApJ 361, L69

Smith V.V., Plez B., Lambert D.L., 1995, ApJ 441, 735

Straniero O., Gallino R., Busso M., Chieffi A., Raiteri C.M., Limongi M., Salaris M., 1995, ApJ 440, L85

Straniero O., et al., 1996, in Torino Workshop on Nucleosynthesis of s- and r-Nuclei I (in press)

Thielemann F.-K., 1991, in Nuclei in the Cosmos I, Oberhummer H. and Rolfs C. (eds.). Springer-Verlag, Berlin, p. 171

Vassiliadis E., Wood P.R., 1993, ApJ 413, 641

Vogelaar R.B., 1989, Ph.D. Thesis, California Institute of Technology, California

Wagoner R.V., 1969, ApJS 162, 247

Wannier P.G., Sahai R., 1986, ApJ 311, 335

Wasserburg G.J., Busso M., Gallino R., Raiteri C.M., 1994, ApJ 424, 412

Weidemann V., 1987, A\&A 188, 74 
Weidemann V., Koester D., 1983, A\&A 121, 77

Wiesher M., Gorres J., Thielemann F.-D., 1990, ApJ 363, 340

Wood P.R., 1981, in Physical Processes in Red Giants, Iben I. and Renzini A. (eds.). Dordrecht, Reidel, p. 135

Wood P.R., Bessell M.S., Fox M.W., 1983, ApJ 272, 99

Woosley S.E., Fowler W.A., Holmes J.A., Zimmerman B.A.,
1978, Atomic Data Nucl. Data Tab. 22, 371

Zinner E., Amari S., Lewis R., 1991b, in Meteoritic Society Meeting, Monterey, California, p. 17

Zinner E., Amari S., Anders E., Lewis R., 1991a, Nat 349, 51

Zuckerman B., Dyck H.M., Claussen M.J., 1986, ApJ 304, 401 